\title{
On WKB Theoretic Transformations for Painlevé Transcendents on Degenerate Stokes Segments
}

\author{
by
}

\author{
Kohei IWAKI
}

\begin{abstract}
The WKB theoretic transformation theorem established in [KT2] implies that the first Painlevé equation gives a normal form of Painlevé equations with a large parameter near a simple $P$-turning point. In this paper we extend this result and show that the second Painlevé equation $\left(P_{\mathrm{II}}\right)$ and the third Painlevé equation $\left(P_{\mathrm{III}^{\prime}\left(D_{7}\right)}\right)$ of type $D_{7}$ give a normal form of Painlevé equations on a degenerate $P$-Stokes segment connecting two different simple $P$-turning points and on a degenerate $P$-Stokes segment of loop type, respectively. That is, any 2-parameter formal solution of a Painlevé equation is reduced to a 2-parameter formal solution of $\left(P_{\mathrm{II}}\right)$ or $\left(P_{\mathrm{III}^{\prime}\left(D_{7}\right)}\right)$ on these degenerate $P$-Stokes segments by our transformation.
\end{abstract}

2010 Mathematics Subject Classification: Primary 34M60; Secondary 34M55.

Keywords: exact WKB analysis, Painlevé equations.

\section{§1. Introduction}

Painlevé transcendents are remarkable special functions which appear in many areas of mathematics and physics. These are solutions of certain non-linear ordinary differential equations known as Painlevé equations. Since the work of Painlevé and Gambier there have been many works which investigate mutual relationships (mainly on the formal level) between different Painlevé equations, often called the degeneration or confluence procedure, or (double) scaling limits of Painlevé equations. More recently, relations of solutions of different Painlevé equations have also been discussed: see [Ki1, Ki2, KapKi, KiVa, Ki3, GIL] and references therein. For example, [KapKi] describes solutions of the first Painlevé equation in terms of those of the second Painlevé equation using infinite iteration of Bäcklund transformations. [GIL] also succeeds in giving a relation between solutions of different

Communicated by S. Mochizuki. Received December 5, 2013. Revised July 19, 2014.

K. Iwaki: Research Institute for Mathematical Sciences, Kyoto University, Kyoto 606-8502, Japan;

e-mail: iwaki@kurims.kyoto-u.ac.jp 


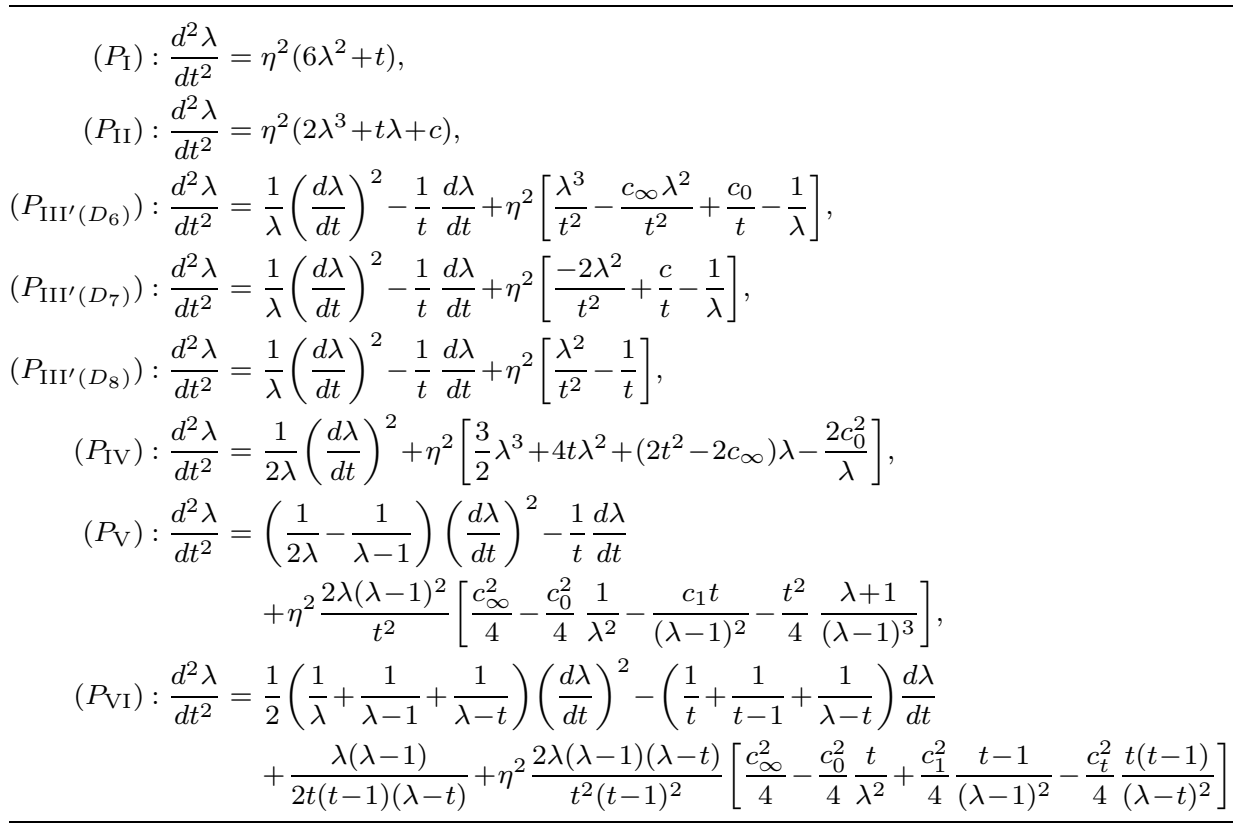

Table 1. Painlevé equations with a large parameter $\eta$.

Painlevé equations through explicit expressions of $\tau$-functions and computations of the limit in the degeneration procedure.

In this paper we discuss a different kind of relations between solutions of Painlevé equations containing a large parameter $\eta$ (cf. Table 1), called a WKB theoretic transformation. It is an invertible formal coordinate transformation which relates formal solutions of different Painlevé equations. (See a series of papers [KT1], [AKT2] and [KT2] by Aoki, Kawai and Takei for more details on WKB theoretic transformations.) The main result of this paper is the construction of new WKB theoretic transformations. That is, for any "2-parameter (formal) solution" of a general Painlevé equation $\left(P_{J}\right)$, we can find a formal invertible coordinate transformation which reduces the 2-parameter solution to a 2-parameter solution of $\left(P_{\mathrm{II}}\right)$ or $\left(P_{\mathrm{III}^{\prime}\left(D_{7}\right)}\right)$, when the configuration of " $P$-Stokes curves" of $\left(P_{J}\right)$ degenerates and contains a $P$-Stokes curve connecting two " $P$-turning points" (we call such a special $P$-Stokes curve a $P$-Stokes segment).

We explain the motivation of our study. Some of the important results by Aoki, Kawai and Takei are summarized as follows (see [KT1], [AKT2] and [KT2]):

- notions of P-turning points and P-Stokes curves are introduced for $\left(P_{J}\right)$,

- 2-parameter (formal) solutions $\lambda_{J}(t, \eta ; \alpha, \beta)$ of $\left(P_{J}\right)$ containing two free parameters $\alpha$ and $\beta$ are constructed by the multiple-scale method, 
- the WKB theoretic transformation theory near a simple P-turning point is established, that is, any 2-parameter solution of $\left(P_{J}\right)$ can be reduced to that of the first Painlevé equation

$$
\left(P_{\mathrm{I}}\right): \frac{d^{2} \lambda}{d t^{2}}=\eta^{2}\left(6 \lambda^{2}+t\right)
$$

on a $P$-Stokes curve emanating from a simple $P$-turning point.

In this paper, for the sake of clarity, we call turning points (resp., Stokes curves) of Painlevé equations $P$-turning points (resp., P-Stokes curves), following the terminology used in [KT4] for example. The precise statement of the last claim is that, for any 2-parameter solution $\tilde{\lambda}_{J}(\tilde{t}, \eta ; \tilde{\alpha}, \tilde{\beta})$ of $\left(P_{J}\right)$, there exist formal coordinate transformation series $x(\tilde{x}, \tilde{t}, \eta)$ and $t(\tilde{t}, \eta)$ of dependent and independent variables and a 2-parameter solution $\lambda_{\mathrm{I}}(t, \eta ; \alpha, \beta)$ of $\left(P_{\mathrm{I}}\right)$ such that

$$
x\left(\tilde{\lambda}_{J}(\tilde{t}, \eta ; \tilde{\alpha}, \tilde{\beta}), \tilde{t}, \eta\right)=\lambda_{\mathrm{I}}(t(\tilde{t}, \eta), \eta ; \alpha, \beta)
$$

in a neighborhood of a point $\tilde{t}=\tilde{t}_{*}$ which lies on a $P$-Stokes curve emanating from a simple $P$-turning point. Here we put $\sim$ on the variables relevant to $\left(P_{J}\right)$ to distinguish them from those of $\left(P_{\mathrm{I}}\right)$. In this sense the first Painlevé equation $\left(P_{\mathrm{I}}\right)$ is a canonical equation for Painlevé equations near a simple $P$-turning point.

The above result can be considered as a non-linear analogue of the transformation theory of linear ordinary differential equations near a simple turning point. In the case of linear equations of second order, a canonical equation is given by the Airy equation:

$$
\left(\frac{d^{2}}{d x^{2}}-\eta^{2} x\right) \psi(x, \eta)=0 .
$$

See $[\mathrm{AKT} 1]$ for the precise statement. The transformation gives an equivalence between $W K B$ solutions of a general Schrödinger equation and those of the Airy equation (1.3) near a simple turning point, and consequently the explicit form of the connection formula on a Stokes curve for a general equation is determined in a "generic" situation [KT3].

The above genericity assumption means that the Stokes graph of the equation does not contain any (degenerate) Stokes segments (i.e., Stokes curves connecting simple turning points). We say that the Stokes geometry degenerates if such a Stokes segment appears. When a Stokes segment appears in the Stokes geometry, the connection formula does not make sense on the Stokes segment (cf. [V, Section 7]).

Typically two types of Stokes segments appear in the Stokes geometry of linear equations: A Stokes segment of the first type connects two different simple turning points, while a Stokes segment of the second type (sometimes called a loop-type 
Stokes segment) emanates from and returns to the same simple turning point and hence forms a closed loop.

To analyze the degenerate situation where a Stokes segment connects two different simple turning points for a general Schrödinger equation, [AKT3] constructs a transformation which brings WKB solutions of the general equation to that of the Weber equation when $x$ lies on a Stokes segment. Here the Weber equation they discuss has the form

$$
\left(\frac{d^{2}}{d x^{2}}-\eta^{2}\left(c-\frac{x^{2}}{4}\right)\right) \psi(x, \eta)=0 .
$$

To be more precise, we need to replace the constant $c$ by a formal power series $c=c(\eta)$ in $\eta^{-1}$ with constant coefficients when discussing the transformation. The Stokes geometry of the equation (1.4) when $c \in \mathbb{R}_{\neq 0}$ (where $\mathbb{R}_{\neq 0}$ is the set of non-zero real numbers) has two simple turning points connected by a Stokes segment. In this sense the Weber equation gives a canonical equation on a Stokes segment which connects two different simple turning points.

On the other hand, recently Takahashi [Ta] has constructed a similar kind of formal transformation which brings a general Schrödinger equation having a loop-type Stokes segment to the Bessel-type equation of the form

$$
\left(\frac{d^{2}}{d x^{2}}-\eta^{2}\left(\frac{x-c^{2}}{x^{2}}\right)\right) \psi(x, \eta)=0 .
$$

When $c \in \mathbb{R}_{\neq 0}$, the Stokes geometry of (1.5) has one simple turning point, and a Stokes curve emanating from the turning point turns around the double pole $x=0$ of the potential and returns to the original simple turning point. This gives a loop-type Stokes segment. In this sense the Bessel-type equation gives a canonical equation on a loop-type Stokes segment.

The transformations constructed in [AKT3] and [Ta] are expected to play important roles in the analysis of parametric Stokes phenomena. Actually, if we vary the constant $c$, WKB solutions of (1.4) may enjoy a Stokes phenomenon, that is, the correspondence between WKB solutions and their Borel sums changes discontinuously before and after the appearance of Stokes segments (cf. [SS], [T3]). We call such Stokes phenomena "parametric" since the Stokes phenomena occur when we vary the parameter $c$ which is not an independent variable. Due to parametric Stokes phenomena, the transformation to the Airy equation does not work when a Stokes segment appears. Actually, a Stokes segment yields so-called fixed singularities (cf. [DP], [AKT3]) for the Borel transform of WKB solutions. Parametric Stokes phenomena are caused by such singularities. They are analysed in [AKT3] through the transformation to the Weber equation. If the Borel summability of the 
transformation series constructed in [AKT3] and [Ta] is established, then the explicit form of the connection formula describing the parametric Stokes phenomena will be derived.

Here a natural question arises: What happens to 2-parameter solutions of $\left(P_{J}\right)$ when the $P$-Stokes geometry degenerates, that is, when a $P$-Stokes segment appears in the $P$-Stokes geometry of $\left(P_{J}\right)$ ?

It is shown in the author's papers [Iw1]-[Iw3] that the parametric Stokes phenomena also occur for 1-parameter solutions (which belongs to a subclass of 2-parameter solutions) of the Painlevé equations when a $P$-Stokes segment appears. For example, when the parameter $c$ contained in the second Painlevé equation

$$
\left(P_{\mathrm{II}}\right): \frac{d^{2} \lambda}{d t^{2}}=\eta^{2}\left(2 \lambda^{3}+t \lambda+c\right)
$$

is purely imaginary, $P$-Stokes segments appear in the $P$-Stokes geometry of $\left(P_{\mathrm{II}}\right)$. In this case three $P$-Stokes segments appear simultaneously and each connects two different simple $P$-turning points (see Section 3.3). It is shown in [Iw1] that 1-parameter solutions of $\left(P_{\mathrm{II}}\right)$ enjoy Stokes phenomena when $P$-Stokes segments appear. Similarly, a loop-type $P$-Stokes segment also appears in the $P$-Stokes geometry of the degenerate third Painlevé equation

$$
\left(P_{\mathrm{III}^{\prime}\left(D_{7}\right)}\right): \frac{d^{2} \lambda}{d t^{2}}=\frac{1}{\lambda}\left(\frac{d \lambda}{d t}\right)^{2}-\frac{1}{t} \frac{d \lambda}{d t}+\eta^{2}\left[\frac{-2 \lambda^{2}}{t^{2}}+\frac{c}{t}-\frac{1}{\lambda}\right]
$$

of type $D_{7}$ (in the sense of [OKSO]) when $c \in i \mathbb{R}_{\neq 0}$ (see Section 3.3).

Motivated by these results, in this paper we construct a transformation of the form (1.2) when the $P$-Stokes geometry of $\left(P_{J}\right)$ degenerates. That is, as is described below, (under some geometric assumptions on the Stokes geometry of isomonodromy systems) when a $P$-Stokes segment which connects two different simple $P$-turning points (resp., a loop-type $P$-Stokes segment) appears, then any 2-parameter solution of $\left(P_{J}\right)$ is reduced to a 2-parameter solution of $\left(P_{\mathrm{II}}\right)$ (resp., $\left.\left(P_{\mathrm{III}\left(D_{7}\right)}\right)\right)$ on the $P$-Stokes segment (see Sections 4 and 5 for precise statements and assumptions).

Theorem 1.1 (Theorem 4.2). Assume that $\left(P_{J}\right)$ has a P-Stokes segment connecting two different simple $P$-turning points of $\left(P_{J}\right)$. Then, for any 2-parameter solution $\tilde{\lambda}_{J}(\tilde{t}, \eta ; \tilde{\alpha}, \tilde{\beta})$ of $\left(P_{J}\right)$, we can find

- formal coordinate transformation series $x(\tilde{x}, \tilde{t}, \eta)$ and $t(\tilde{t}, \eta)$ of dependent and independent variables,

- a 2-parameter solution $\lambda_{\mathrm{II}}(t, \eta ; \alpha, \beta)$ of $\left(P_{\mathrm{II}}\right)$ with a suitable choice of the constant $c$ in the equation, 
satisfying

$$
x\left(\tilde{\lambda}_{J}(\tilde{t}, \eta ; \tilde{\alpha}, \tilde{\beta}), \tilde{t}, \eta\right)=\lambda_{\mathrm{II}}(t(\tilde{t}, \eta), \eta ; \alpha, \beta)
$$

in a neighborhood of a point $\tilde{t}=\tilde{t}_{*}$ which lies on the P-Stokes segment.

Theorem 1.2 (Theorem 5.2). Assume that $\left(P_{J}\right)$ has a P-Stokes segment of loop type. Then, for any 2-parameter solution $\tilde{\lambda}_{J}(\tilde{t}, \eta ; \tilde{\alpha}, \tilde{\beta})$ of $\left(P_{J}\right)$, we can find

- formal coordinate transformation series $x(\tilde{x}, \tilde{t}, \eta)$ and $t(\tilde{t}, \eta)$ of dependent and independent variables,

- a 2-parameter solution $\lambda_{\mathrm{III}^{\prime}\left(D_{7}\right)}(t, \eta ; \alpha, \beta)$ of $\left(P_{\mathrm{III}^{\prime}\left(D_{7}\right)}\right)$ with a suitable choice of the constant $c$,

satisfying

$$
x\left(\tilde{\lambda}_{J}(\tilde{t}, \eta ; \tilde{\alpha}, \tilde{\beta}), \tilde{t}, \eta\right)=\lambda_{\mathrm{III}^{\prime}\left(D_{7}\right)}(t(\tilde{t}, \eta), c, \eta ; \alpha, \beta)
$$

in a neighborhood of a point $\tilde{t}=\tilde{t}_{*}$ which lies on the P-Stokes segment of loop type.

In this sense, $\left(P_{\mathrm{II}}\right)$ and $\left(P_{\mathrm{III}^{\prime}\left(D_{7}\right)}\right)$ are canonical equations for Painlevé equations on a $P$-Stokes segment connecting different simple $P$-turning points and a loop-type $P$-Stokes segment, respectively. Our main results can be considered to be non-linear analogues of the transformation theory of [AKT3] (to the Weber equation) and [Ta] (to the Bessel-type equation). We expect that, together with the previous results [Iw1]-[Iw3], our transformation theory will play an important role in the analysis of parametric Stokes phenomena for Painlevé equations.

This paper is organized as follows. In Section 2 we briefly review some results on WKB analysis of Painlevé equations $\left(P_{J}\right)$ and the role of isomonodromy systems $\left(S L_{J}\right)$ and $\left(D_{J}\right)$ associated with $\left(P_{J}\right)$. Section 3 is devoted to the $P$-Stokes geometry of $\left(P_{J}\right)$ and the Stokes geometry of $\left(S L_{J}\right)$. Our main assumptions and results are stated and proved in Sections 4 and 5 .

\section{$\S 2$. Review of the exact WKB analysis of Painlevé transcendents with a large parameter}

In this section we prepare some notation and review some results of [KT1], [AKT2] and $[\mathrm{KT} 2]$ that are relevant to this paper.

$\S 2.1$ 2-parameter solution $\lambda_{J}(t, \eta ; \alpha, \beta)$ of $\left(P_{J}\right)$

In [AKT2] a 2-parameter family of formal solutions of $\left(P_{J}\right)$, called a 2-parameter solution, is constructed by the so-called multiple-scale method. Here we introduce 
some notation to describe the solutions explicitly and to make our discussion smooth. The notation introduced here is mostly consistent with that of [KT2].

As is clear from Table 1, each $\left(P_{J}\right)$ has the form

$$
\left(P_{J}\right): \frac{d^{2} \lambda}{d t^{2}}=G_{J}\left(\lambda, \frac{d \lambda}{d t}, t\right)+\eta^{2} F_{J}(\lambda, t)
$$

where $F_{J}$ is a rational function in $t$ and $\lambda$, and $G_{J}$ is a polynomial in $d \lambda / d t$ with degree equal to or at most 2, and rational in $\lambda$ and $t$. Define the set $\operatorname{Sing}_{J} \subset \mathbb{P}^{1}$ of singular points of $\left(P_{J}\right)$ by

$$
\begin{aligned}
& \operatorname{Sing}_{\mathrm{I}}=\operatorname{Sing}_{\mathrm{II}}=\{\infty\}, \\
& \operatorname{Sing}_{\mathrm{III}\left(D_{6}\right)}=\operatorname{Sing}_{\mathrm{III}\left(D_{7}\right)}=\operatorname{Sing}_{\mathrm{III}\left(D_{8}\right)}=\{0, \infty\}, \\
& \operatorname{Sing}_{\mathrm{IV}}=\{\infty\}, \quad \operatorname{Sing}_{\mathrm{V}}=\{0, \infty\}, \quad \operatorname{Sing}_{\mathrm{VI}}=\{0,1, \infty\},
\end{aligned}
$$

and the set $\Delta_{J}$ of branch points of $\left(P_{J}\right)$ by

$$
\Delta_{J}=\left\{r \in \mathbb{P}^{1} \backslash \operatorname{Sing}_{J} \mid F_{J}(\lambda, r)=\left(\partial F_{J} / \partial \lambda\right)(\lambda, r)=0 \text { for some } \lambda\right\} .
$$

We also set $\Omega_{J}=\mathbb{P}^{1} \backslash\left(\operatorname{Sing}_{J} \cup \Delta_{J}\right)$.

Fix a holomorphic function $\lambda_{0}(t)$ that satisfies

$$
F_{J}\left(\lambda_{0}(t), t\right)=0
$$

near a point $t_{*} \in \Omega_{J}$. The 2-parameter solutions are formal solutions of $\left(P_{J}\right)$ defined in a neighborhood $V$ of $t_{*}$ of the form

$$
\lambda_{J}(t, \eta ; \alpha, \beta)=\lambda_{0}(t)+\eta^{-1 / 2} \sum_{j=0}^{\infty} \eta^{-j / 2} \Lambda_{j / 2}(t, \eta ; \alpha, \beta) .
$$

Here $(\alpha, \beta)=\left(\sum_{n=0}^{\infty} \eta^{-n} \alpha_{n}, \sum_{n=0}^{\infty} \eta^{-n} \beta_{n}\right)$ is a pair of formal power series whose coefficients $\left\{\left(\alpha_{n}, \beta_{n}\right)\right\}_{n=0}^{\infty}$ parametrize the formal solution, and the functions

$$
\Lambda_{j / 2}(t, \eta ; \alpha, \beta)=\sum_{m=0}^{j+1} a_{j+1-2 m}^{(j / 2)}(t) \exp \left((j+1-2 m) \Phi_{J}(t, \eta)\right)
$$

labeled by half-integers have the following properties (see [AKT2], [KT2]):

- For any $j \geq 0$ and $\ell=j+1-2 m(m=0, \ldots, j+1), a_{\ell}^{(j / 2)}(t)$ is a holomorphic function of $t$ on $V$ and independent of $\eta$.

- The functions $a_{ \pm 1}^{(0)}(t)$ contain the free parameters $\left(\alpha_{0}, \beta_{0}\right)$ as

$$
a_{+1}^{(0)}(t)=\frac{\alpha_{0}}{\sqrt[4]{F_{J}^{(1)}(t) C_{J}\left(\lambda_{0}(t), t\right)^{2}}}, \quad a_{-1}^{(0)}(t)=\frac{\beta_{0}}{\sqrt[4]{F_{J}^{(1)}(t) C_{J}\left(\lambda_{0}(t), t\right)^{2}}},
$$


where

$$
F_{J}^{(1)}(t)=\frac{\partial F_{J}}{\partial \lambda}\left(\lambda_{0}(t), t\right)
$$

and $C_{J}(\lambda, t)$ is given in Table 2 .

$$
\begin{gathered}
C_{\mathrm{I}}(\lambda, t)=C_{\mathrm{II}}(\lambda, t)=1, \quad C_{\mathrm{III}^{\prime}\left(D_{6}\right)}(\lambda, t)=C_{\mathrm{III}^{\prime}\left(D_{7}\right)}(\lambda, t)=C_{\mathrm{III}^{\prime}\left(D_{8}\right)}(\lambda, t)=\frac{t}{2 \lambda^{2}}, \\
C_{\mathrm{IV}}(\lambda, t)=\frac{1}{4 \lambda}, \quad C_{\mathrm{V}}(\lambda, t)=\frac{t}{2 \lambda(\lambda-1)^{2}}, \quad C_{\mathrm{VI}}(\lambda, t)=\frac{t(t-1)}{2 \lambda(\lambda-1)(\lambda-t)}
\end{gathered}
$$

Table 2. $C_{J}(\lambda, t)$.

- The function $\Phi_{J}(t, \eta)$, which is also holomorphic in $t \in V$, is given by

$$
\Phi_{J}(t, \eta)=\eta \phi_{J}(t)+\alpha_{0} \beta_{0} \log \left(\theta_{J}(t) \eta^{2}\right),
$$

where

$$
\phi_{J}(t)=\int^{t} \sqrt{F_{J}^{(1)}(t)} d t
$$

and $\theta_{J}(t)$ is determined from $F_{J}, G_{J}$ and $\lambda_{0}(t)$ (cf. [KT2, Section 1]). We will fix the lower end-point of the integration path later.

- The functions $a_{\ell}^{(j / 2)}(t)$ and $(\ell \neq \pm 1)$ are determined recursively from $\left\{a_{j^{\prime}+1-2 m}^{\left(j^{\prime} / 2\right)}(t)\right\}_{j^{\prime}<j, 0 \leq m \leq j^{\prime}+1}$.

- $a_{ \pm 1}^{(j / 2)}(t)=0$ for $j$ odd, while $a_{+1}^{(j / 2)}(t)$ and $a_{-1}^{(j / 2)}(t)$ for $j \geq 2$ even satisfy a system of linear inhomogeneous differential equations of the form

$$
\begin{aligned}
\left\{\frac{d}{d t}+\frac{1}{4} \frac{d}{d t}\right. & \log F_{J}^{(1)}(t)+\frac{1}{2} \log C_{J}\left(\lambda_{0}(t), t\right) \\
& \left.-\left(\begin{array}{cc}
\alpha_{0} \beta_{0} & \alpha_{0}^{2} \\
-\beta_{0}^{2} & -\alpha_{0} \beta_{0}
\end{array}\right) \frac{d}{d t} \log \theta_{J}(t)\right\}\left(\begin{array}{c}
a_{+1}^{(n)} \\
a_{-1}^{(n)}
\end{array}\right)=\left(\begin{array}{c}
R_{+1}^{(n)} \\
R_{-1}^{(n)}
\end{array}\right),
\end{aligned}
$$

where $R_{ \pm 1}^{(n)}$ is determined by $\left\{a_{j^{\prime}+1-2 m}^{\left(j^{\prime} / 2\right)}(t)\right\}_{j^{\prime}<j=2 n, 0 \leq m \leq j^{\prime}+1}$. The free parameters $\left(\alpha_{n}, \beta_{n}\right)(n \geq 1$ integer) capture the ambiguity of solutions of the differential equation for $j=2 n$.

Therefore, 2-parameter solutions are formal power series in $\eta^{-1 / 2}$ whose coefficients $\Lambda_{j / 2}$ may contain $\eta$-dependent terms of the form $\exp (\ell \Phi(t, \eta))$ for some $\ell \in \mathbb{Z}$, called $\ell$-instanton terms in [KT2]. In the present paper, formal series means such a series, and we say that $\Lambda_{j / 2}(t, \eta)$ is holomorphic in $t$ if the coefficients of each instanton term in $\Lambda_{j / 2}(t, \eta)$ are holomorphic in $t$. Note that $\Lambda_{j / 2}(t, \eta)$ contains instanton terms in such a way that, if $j$ is odd (resp., even), then $\Lambda_{j / 2}(t, \eta)$ 


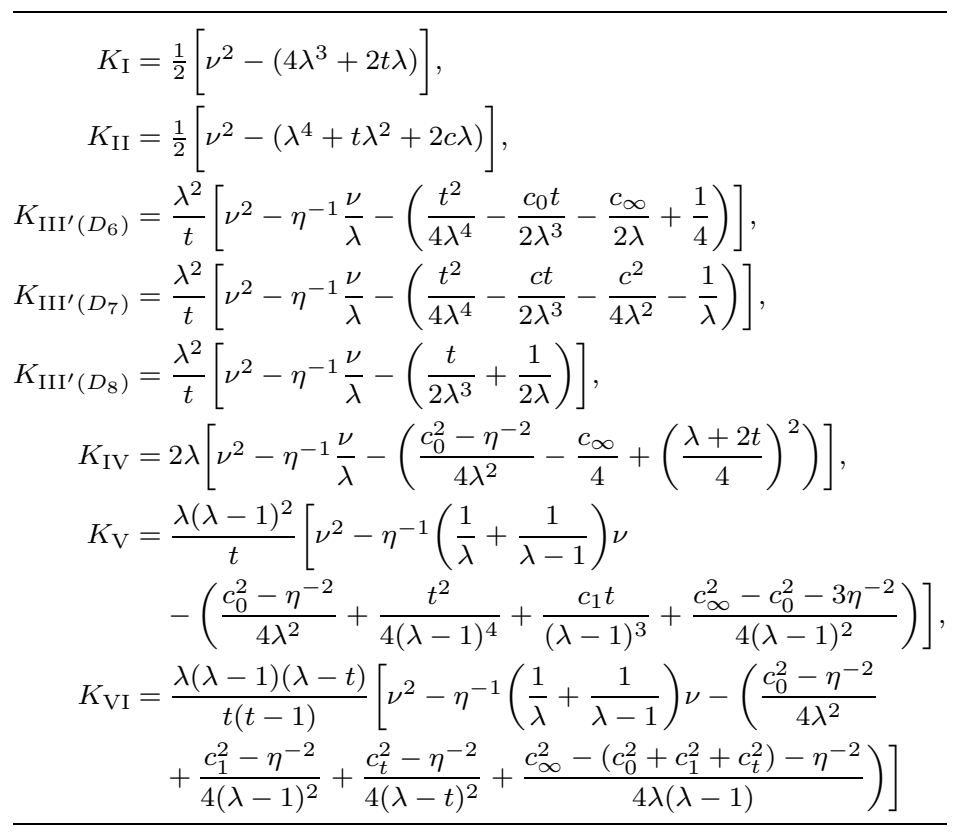

Table 3. Hamiltonians of $\left(H_{J}\right)$.

contains only even (resp., odd) instanton terms. We call this property the alternating parity of 2-parameter solutions. In order to avoid some degeneracy, we assume throughout that

$$
\alpha_{0} \beta_{0} \neq 0 \text {. }
$$

It is well-known that the Painlevé equation $\left(P_{J}\right)$ is equivalent to the following Hamiltonian system (see, e.g., [Ok]):

$$
\left(H_{J}\right): \frac{d \lambda}{d t}=\eta \frac{\partial K_{J}}{\partial \nu}, \quad \frac{d \nu}{d t}=-\eta \frac{\partial K_{J}}{\partial \lambda} .
$$

Here the explicit forms of the Hamiltonians $K_{J}=K_{J}(t, \lambda, \nu, \eta)$ are tabulated in Table 3. From 2-parameter solutions of $\left(P_{J}\right)$, we can also construct 2-parameter solutions of the Hamiltonian system $\left(H_{J}\right)$. From the explicit form of the Hamiltonians $K_{J}$, we see that $\nu$ is determined by $\lambda$ and its first order derivative. Consequently, $\nu_{J}=\nu_{J}(t, \eta ; \alpha, \beta)$ has the form

$$
\nu_{J}(t, \eta ; \alpha, \beta)=\eta^{-1 / 2} \sum_{j=0}^{\infty} \eta^{-j / 2} N_{j / 2}(t, \eta ; \alpha, \beta),
$$

where $N_{j / 2}$ has a similar form to $\Lambda_{j / 2}$, that is, it contains instanton terms and exhibits alternating parity. 
Remark 2.1. If we put $\alpha=0$ or $\beta=0$ or $\alpha=\beta=0$, then 2-parameter solutions reduce to 1-parameter solutions or 0-parameter solutions. Here $\alpha=0$ etc. mean that all $\alpha_{n}$ are set to be 0 etc.; and 1-parameter solutions are also called trans-series solutions. We can expect that 1-parameter solutions and 0-parameter solutions are interpreted as analytic solutions through the Borel resummation method (see [KaKo] for example).

\section{§2.2. Isomonodromy system for $\left(P_{J}\right)$ and WKB solutions}

The Hamiltonian system $\left(H_{J}\right)$ arises when we consider isomonodromic deformations (see [JMU], [Ok]) of a Schrödinger equation of the form

$$
\left(S L_{J}\right):\left(\frac{\partial^{2}}{\partial x^{2}}-\eta^{2} Q_{J}(x, t, \eta)\right) \psi(x, t, \eta)=0 .
$$

More precisely, there exists another differential equation

$$
\left(D_{J}\right): \frac{\partial}{\partial t} \psi(x, t, \eta)=\left(A_{J}(x, t, \eta) \frac{\partial}{\partial x}-\frac{1}{2} \frac{\partial A_{J}}{\partial x}(x, t, \eta)\right) \psi(x, t, \eta),
$$

called the deformation equation, such that $\left(H_{J}\right)$ gives the compatibility conditions for the system of linear differential equations $\left(S L_{J}\right)$ and $\left(D_{J}\right)$. See Tables 4 and 5 for the explicit forms of $Q_{J}$ and $A_{J}$.

Substituting 2-parameter solutions $\left(\lambda_{J}(t, \eta ; \alpha, \beta), \nu_{J}(t, \eta ; \alpha, \beta)\right)$ into $(\lambda, \nu)$ that appears in $Q_{J}$ and $A_{J}$, we find that they have the same type formal series expansions

$$
Q_{J}(x, t, \eta)=\sum_{j=0}^{\infty} Q_{j / 2}(x, t, \eta), \quad A_{J}(x, t, \eta)=\sum_{j=0}^{\infty} A_{j / 2}(x, t, \eta) .
$$

Here we omit indicating explicitly the dependence on $\alpha$ and $\beta$ for simplicity. The top term $Q_{0}=Q_{J, 0}(x, t)$ is independent of $\eta$ (i.e., it does not contain instanton terms), and can be written in the form

$$
Q_{J, 0}(x, t)=C_{J}(x, t)^{2}\left(x-\lambda_{0}(t)\right)^{2} R_{J}(x, t) .
$$

Thus, $Q_{J, 0}(x, t)$ has a double zero at $x=\lambda_{0}(t)$ in general. (Here we have used the fact that $\lambda_{0}(t)$ is defined by the algebraic equation (2.3).) Here $R_{J}(x, t)$ is a polynomial in $x$ which satisfies

$$
R_{J}\left(\lambda_{0}(t), t\right)=F_{J}^{(1)}(t) .
$$

We can verify that $R_{\mathrm{I}}(x, t), R_{\mathrm{III}^{\prime}\left(D_{7}\right)}(x, t)$ and $R_{\mathrm{III}^{\prime}\left(D_{8}\right)}(x, t)$ are polynomial in $x$ of degree 1 , while $R_{J}(x, t)$ for other $J$ are polynomial in $x$ of degree 2 . 


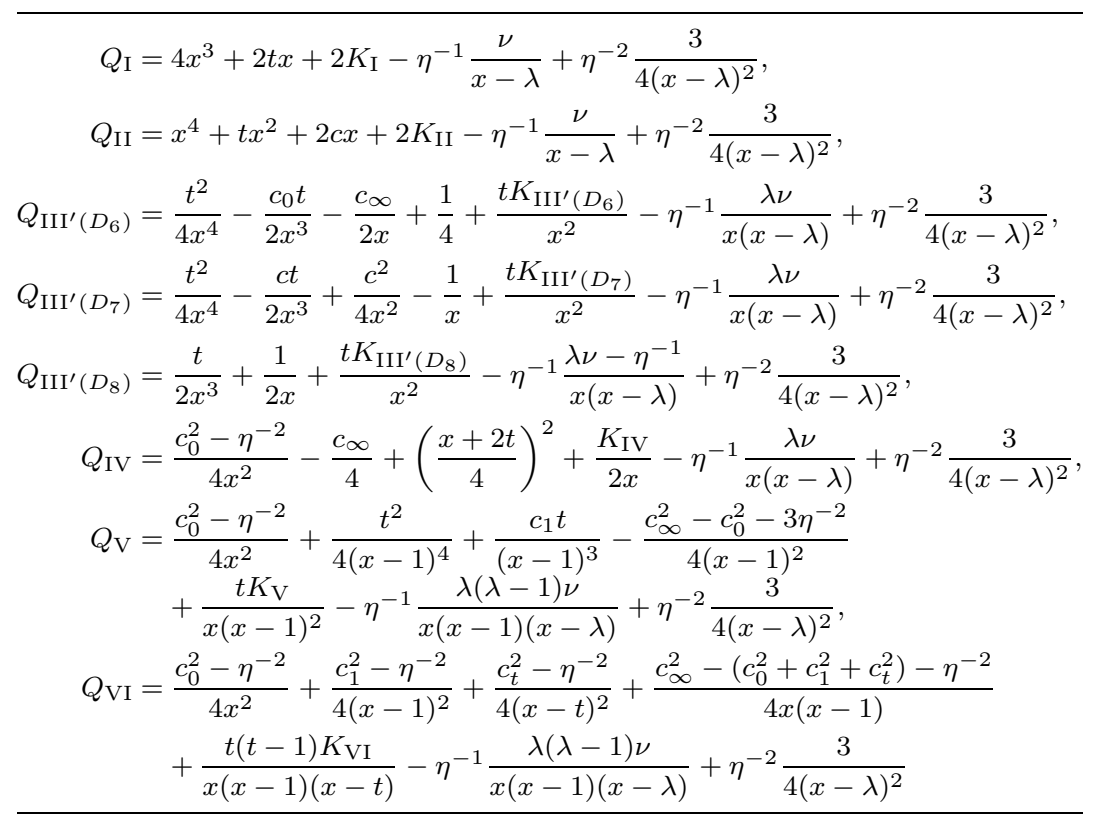

Table 4. Coefficient of $\left(S L_{J}\right)$.

$A_{\mathrm{I}}=A_{\mathrm{II}}=\frac{1}{2(x-\lambda)}, \quad A_{\mathrm{III}\left(D_{6}\right)}=A_{\mathrm{III}^{\prime}\left(D_{7}\right)}=A_{\mathrm{III}^{\prime}\left(D_{8}\right)}=\frac{\lambda x}{t(x-\lambda)}$,
$A_{\mathrm{IV}}=\frac{2 x}{x-\lambda}, \quad A_{\mathrm{V}}=\frac{\lambda-1}{t} \frac{x(x-1)}{x-\lambda}, \quad A_{\mathrm{VI}}=\frac{\lambda-t}{t(t-1)} \frac{x(x-1)}{x-\lambda}$

Table 5. Coefficient of $\left(D_{J}\right)$.

In what follows, we always assume that a 2-parameter solution $\left(\lambda_{J}, \nu_{J}\right)$ of $\left(H_{J}\right)$ is substituted into $(\lambda, \nu)$ which appears in the coefficients of $\left(S L_{J}\right)$ and $\left(D_{J}\right)$. For such a Schrödinger equation $\left(S L_{J}\right)$, we can construct WKB solutions of the form

$$
\psi_{J, \pm}(x, t, \eta)=\frac{1}{\sqrt{S_{J, \text { odd }}(x, t, \eta)}} \exp \left( \pm \int^{x} S_{J, \text { odd }}(x, t, \eta) d x\right) .
$$

Here $S_{J, \text { odd }}(x, t, \eta)$ is the odd part of a formal series solution $S_{J}(x, t, \eta)$ of

$$
S^{2}+\frac{\partial S}{\partial x}=\eta^{2} Q_{J}(x, t, \eta)
$$

which is called the Riccati equation associated with $\left(S L_{J}\right)$. Here the odd part $S_{J, \text { odd }}(x, t, \eta)$ is defined as follows (see [AKT2] for details). We can find two formal 
series solutions

$$
S_{J}^{( \pm)}(x, t, \eta)=\eta S_{-1}^{( \pm)}(x, t)+\sum_{j=0}^{\infty} \eta^{-j / 2} S_{j / 2}^{( \pm)}(x, t, \eta)
$$

of (2.17) starting from

$$
S_{-1}^{( \pm)}(x, t)= \pm \sqrt{Q_{J, 0}(x, t)} .
$$

Once we fix the sign in (2.19) (i.e., the branch of the square root), the subsequent terms are determined by a recursion relation. Then $S_{J, \text { odd }}(x, t, \eta)$ is given by

$$
\begin{aligned}
S_{J, \text { odd }}(x, t, \eta) & =\frac{1}{2}\left(S_{J}^{(+)}(x, t, \eta)-S_{J}^{(-)}(x, t, \eta)\right) \\
& =\eta S_{-1}(x, t)+\sum_{j=0}^{\infty} \eta^{-j / 2} S_{\text {odd }, j / 2}(x, t, \eta) .
\end{aligned}
$$

The integral of $S_{J, \text { odd }}(x, t, \eta)$ appearing in (2.16) is defined by termwise integration of formal series. We discuss the choice of the lower end-point of (2.16) later.

The formal series $S_{J, \text { odd }}(x, t, \eta)$ etc. are constructed in the above manner for a fixed $t$ and have several good properties as functions of $t$. Firstly, $S_{J \text {,odd }}(x, t, \eta)$ also has the property of alternating parity: if $j$ is odd (resp., even), then $S_{\text {odd }, j / 2}(x, t, \eta)$ contains only odd (resp., even) instanton terms. Secondly, the derivative of $S_{J, \text { odd }}(x, t, \eta)$ with respect to $t$ satisfies the following equation.

Proposition 2.2 ([AKT2, Proposition 2.1]). The formal solutions $S_{J}^{( \pm)}(x, t, \eta)$ satisfy

$$
\frac{\partial}{\partial t} S_{J}^{( \pm)}(x, t, \eta)=\frac{\partial}{\partial x}\left(S_{J}^{( \pm)}(x, t, \eta) A_{J}(x, t, \eta)-\frac{1}{2} \frac{\partial A_{J}}{\partial x}(x, t, \eta)\right)
$$

and hence

$$
\frac{\partial}{\partial t} S_{J, \text { odd }}(x, t, \eta)=\frac{\partial}{\partial x}\left(S_{J, \text { odd }}(x, t, \eta) A_{J}(x, t, \eta)\right)
$$

Proposition 2.2 is proved by using the isomonodromy property of $\left(S L_{J}\right)$, that is, the compatibility of $\left(S L_{J}\right)$ and $\left(D_{J}\right)$. As a corollary, we obtain the following important (formal series valued) first integral of $\left(P_{J}\right)$ from $\left(S L_{J}\right)$.

Lemma 2.3 ([AKT2, Section 3]). The formal series $E(\eta)$ defined by

$$
E_{J}(\eta)=4 \operatorname{Res}_{x=\lambda_{0}(t)} S_{J, \text { odd }}(x, t, \eta) d x
$$

is independent of $t$. 
The independence from $t$ implies that $E_{J}(\eta)$ must be a formal power series in $\eta^{-1}$, that is, $E_{J}(\eta)=\sum_{n=0}^{\infty} \eta^{-n} E_{n}$ with some constants $E_{n}$. The free parameters $\alpha_{n}$ and $\beta_{n}$ of a 2-parameter solution are contained in $E_{n}$ in the following manner.

Lemma 2.4 ([KT2, Lemma 3.2]). (i) The top term $E_{0}$ of $E_{J}(\eta)$ is given by

$$
E_{0}=-8 \alpha_{0} \beta_{0}
$$

(ii) The coefficient $E_{n}$ of $\eta^{-n}$ in $E_{J}(\eta)$ depends only on $\left\{\alpha_{n^{\prime}}, \beta_{n^{\prime}}\right\}_{0 \leq n^{\prime} \leq n}$. Furthermore, $E_{n}+8\left(\alpha_{0} \beta_{n}+\alpha_{n} \beta_{0}\right)$ is independent of $\left(\alpha_{n}, \beta_{n}\right)$.

Remark 2.5. Let us take a generic point $t_{*}$ such that $Q_{J, 0}(x, t)$ has a simple zero $x=a(t)$ at any point $t$ in a neighborhood of $t_{*}$. It is known that each coefficient $S_{\text {odd }, j / 2}(x, t, \eta)$ of $S_{J, \text { odd }}(x, t, \eta)$ has a square root type singularity at a simple zero of $Q_{J, 0}$ (see, e.g., [KT3, Section 2]). Due to this property we can define the WKB solution of $\left(S L_{J}\right)$ which is "well-normalized" at $x=a(t)$ as follows:

$$
\psi_{J, \pm}(x, t, \eta)=\frac{1}{\sqrt{S_{J, \text { odd }}(x, t, \eta)}} \exp \left( \pm \int_{a(t)}^{x} S_{J, \text { odd }}(x, t, \eta) d x\right) .
$$

Here the integral in (2.25) is defined as a contour integral, that is,

$$
\int_{a(t)}^{x} S_{J, \text { odd }}(x, t, \eta) d x=\frac{1}{2} \int_{\delta_{x}} S_{J, \text { odd }}(x, t, \eta) d x,
$$

where the path $\delta_{x}$ is depicted in Figure 1. The wiggly line in the figure is a branch cut to determine the branch of $\sqrt{Q_{J, 0}(x, t)}$, and the solid (resp., dashed) line is a part of the path $\delta_{x}$ on the first (resp., second) sheet of the Riemann surface of $\sqrt{Q_{J, 0}(x, t)}$. Then, we can show that the well-normalized WKB solutions (2.25) satisfy both $\left(S L_{J}\right)$ and $\left(D_{J}\right)$ by using $(2.22)$ (cf. [T2, Lemma 1]).

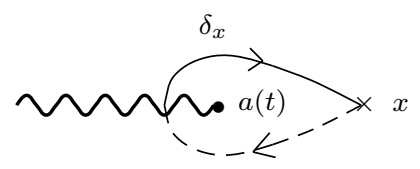

Figure 1. Integration path $\delta_{x}$.

The following proposition will play an important role in the proof of our main theorems.

Proposition 2.6. Let $p$ be an even order pole of $Q_{J, 0}(x, t)$ (hence, a singular point of $\left.\left(S L_{J}\right)\right)$, and set

$$
\operatorname{Res}\left(S L_{J}, p\right)=\operatorname{Res}_{x=p} S_{J, \text { odd }}(x, t, \eta) d x .
$$

Then the list of $\operatorname{Res}\left(S L_{J}, p\right)$ for all $J$ and $p$ is given in Table 6, up to sign. 


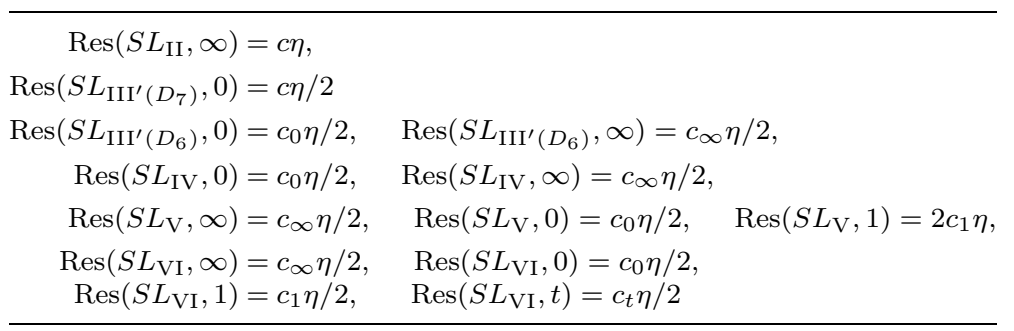

Table 6. $\operatorname{Res}\left(S L_{J}, p\right)$ at singular points of $\left(S L_{J}\right)$.

Proof. Let us show the claim when $J=$ II and $p=\infty$. The coefficients $\left\{S_{j / 2}^{( \pm)}(x, t, \eta)\right\}_{j \geq-2}$ of the formal series $S_{J}^{( \pm)}(x, t, \eta)$ in (2.18) must satisfy the recursion relations

$$
\begin{aligned}
S_{-1}^{( \pm)}(x, t) & = \pm \sqrt{Q_{J, 0}(x, t)}, \quad S_{-1 / 2}^{( \pm)}(x, t)=0 \\
S_{(j+2) / 2}^{( \pm)} & =\frac{1}{2 S_{-1}^{( \pm)}}\left(Q_{(j+4) / 2}-\frac{\partial S_{j / 2}^{( \pm)}}{\partial x}-\sum_{\substack{j_{1}+j_{2}=j \\
0 \leq j_{1}, j_{2} \leq j}} S_{j_{1} / 2}^{( \pm)} S_{j_{2} / 2}^{( \pm)}\right) \quad(j \geq-2)
\end{aligned}
$$

since $S_{J}^{( \pm)}(x, t, \eta)$ solve the Riccati equation (2.17). We can then directly compute the asymptotic behavior of $S_{j / 2}^{( \pm)}(x, t, \eta)$ near $x=\infty$ from the recursion relations (2.27) and (2.28) and the explicit form of the potential $Q_{J}$ in Table 4; for example, when $J=\mathrm{II}$, these are given by

$$
S_{j / 2}^{( \pm)}(x, t, \eta)= \begin{cases} \pm\left(x^{2}+t / 2-c x^{-1}+O\left(x^{-2}\right)\right) & \text { if } j=-2 \\ O\left(x^{-2}\right) & \text { if } j \geq-1\end{cases}
$$

Thus we have

$$
\operatorname{Res}_{x=\infty} S_{\mathrm{II}, \mathrm{odd}}(x, t, \eta) d x= \pm c \eta \text {. }
$$

In a similar manner we can compute the residues of $S_{J, \text { odd }}(x, t, \eta) d x$ at each singular point for the other $J$ 's by straightforward computations. Actually, when $p$ is a regular singular point of $\left(S L_{J}\right)$, we need a more careful computation since $S_{j / 2}^{( \pm)}(x, t, \eta)$ may have first order poles at regular singular points in view of $(2.27)$ and (2.28). However, by the technique used in the proof of [KT3, Proposition 3.6] we can check that the residues of $S_{j / 2}^{( \pm)}(x, t, \eta) d x$ at regular singular points vanish for $j \geq 0$. Thus we obtain Table 6 .

In particular, we can find that the residues in Table 6 are genuine constants multiplied by $\eta$, which implies that the residue of $S_{J, \text { odd }}(x, t, \eta) d x$ only comes from 
the top term $\eta S_{-1}(x, t) d x$ :

$$
\operatorname{Res}_{x=p} S_{J, \text { odd }}(x, t, \eta) d x=\eta \operatorname{Res}_{x=p} S_{-1}(x, t) d x .
$$

This will simplify our construction of transformations of Painlevé transcendents.

\section{§2.3. Local transformation near the double turning point}

In the (exact) WKB analysis, the zeros of $Q_{J, 0}(x, t)$ play an important role. They are called turning points of $\left(S L_{J}\right)$ (see Definition 3.3 below). In view of $(2.14)$, the point $x=\lambda_{0}\left(t_{*}\right)$ is a double turning point (i.e., a double zero of $Q_{J, 0}\left(x, t_{*}\right)$ ) when $t_{*}$ is a generic point. This double turning point is particularly important in the WKB analysis of Painlevé transcendents.

Let us fix a generic point $t_{*}$ and take a sufficiently small neighborhood $V$ of $t_{*}$ such that $x=\lambda_{0}(t)$ is a double zero of $Q_{J, 0}(x, t)$ at any point $t \in V$. It is shown in [KT2] that the isomonodromy system $\left(S L_{J}\right)$ and $\left(D_{J}\right)$ can be reduced to the system

$$
\begin{aligned}
(\mathrm{Can}) & :\left(\frac{\partial^{2}}{\partial z^{2}}-\eta^{2} Q_{\mathrm{can}}(z, s, \eta)\right) \varphi(z, s, \eta)=0 \\
\left(D_{\mathrm{can}}\right) & : \frac{\partial}{\partial s} \varphi(z, x, \eta)=\left(A_{\mathrm{can}}(z, s, \eta) \frac{\partial}{\partial z}-\frac{\partial A_{\mathrm{can}}}{\partial z}(z, s, \eta)\right) \varphi(z, s, \eta)
\end{aligned}
$$

on $U_{0} \times V$, where $U_{0}$ is a neighborhood of the double turning point $x=\lambda_{0}(t)$. Here $Q_{\text {can }}$ and $A_{\text {can }}$ are given by

$$
\begin{aligned}
Q_{\mathrm{can}}(z, s, \eta)= & 4 z^{2}+\eta^{-1} E(s, \eta) \\
& +\eta^{-1 / 2} \frac{\eta^{-1} \rho(s, \eta)}{z-\eta^{-1 / 2} \sigma(s, \eta)}+\eta^{-2} \frac{3}{4\left(z-\eta^{-1 / 2} \sigma(s, \eta)\right)^{2}} \\
A_{\mathrm{can}}(z, s, \eta)= & \frac{1}{2\left(z-\eta^{-1 / 2} \sigma(s, \eta)\right)}
\end{aligned}
$$

with

$$
E(s, \eta)=\rho(s, \eta)^{2}-4 \sigma(s, \eta)^{2} .
$$

The system (Can) \& $\left(D_{\text {can }}\right)$ is compatible if $\rho$ and $\sigma$ satisfy the Hamiltonian system

$$
\left(H_{\mathrm{can}}\right): \frac{d \rho}{d s}=-4 \eta \sigma, \quad \frac{d \sigma}{d s}=-\eta \rho .
$$

As a solution of $\left(H_{\text {can }}\right)$, we take

$$
\sigma(s, \eta ; A, B)=A e^{2 \eta s}+B e^{-2 \eta s}, \quad \rho(s, \eta ; A, B)=-2 A e^{2 \eta s}+2 B e^{-2 \eta s},
$$


where $A$ and $B$ are complex constants, and (2.34) becomes independent of $s$ :

$$
E(s, \eta ; A, B)=\rho(s, \eta ; A, B)^{2}-4 \sigma(s, \eta ; A, B)^{2}=-16 A B
$$

Denote by $Q_{\text {can }}(z, s, \eta ; A, B)$ the potential (2.32) with the solution $(2.35)$ of $\left(H_{\text {can }}\right)$ substituted into $(\sigma, \rho)$ in its expression. Then the precise statement of the local reduction theorem of $[\mathrm{KT} 2]$ is as follows.

Theorem 2.7 ([KT2, Theorem 2.1, Lemma 3.3]; cf. [AKT2, Theorem 3.1]). Let $t_{*}$ be a generic point as above. Then there exist a neighborhood $U_{0} \times V$ of the point $\left(\lambda_{0}\left(t_{*}\right), t_{*}\right)$ and formal series

$$
\begin{aligned}
z_{J}(x, t, \eta) & =\sum_{j=0}^{\infty} \eta^{-j / 2} z_{j / 2}(x, t, \eta), \\
s_{J}(t, \eta) & =\sum_{j=0}^{\infty} \eta^{-j / 2} s_{j / 2}(t, \eta), \\
A_{J}(\eta) & =\sum_{n=0}^{\infty} \eta^{-n} A_{n}, \quad B_{J}(\eta)=\sum_{n=0}^{\infty} \eta^{-n} B_{n},
\end{aligned}
$$

satisfying the following conditions:

(i) For each $j \geq 0, z_{j / 2}(x, t, \eta)$ and $s_{j / 2}(t, \eta)$ are holomorphic functions in $(x, t) \in U_{0} \times V$ and in $t \in V$, respectively.

(ii) For each $n \geq 0, A_{n}$ and $B_{n}$ are genuine constants.

(iii) $z_{0}(x, t)$ is independent of $\eta, \partial z_{0} / \partial x$ never vanishes on $U_{0} \times V$, and $z_{0}\left(\lambda_{0}(t), t\right)$ $=0$.

(iv) $s_{0}(t)$ is also independent of $\eta$ and $d s_{0} / d t$ never vanishes on $V$.

(v) $z_{1 / 2}(x, t)$ and $s_{1 / 2}(t)$ vanish identically.

(vi) The $\eta$-dependence of $z_{j / 2}(x, t, \eta)$ and $s_{j / 2}(t, \eta)(j \geq 2)$ is only through instanton terms $\exp \left(\ell \Phi_{J}(t, \eta)\right)$ for $\ell=j-2-2 j^{\prime}$ with $0 \leq j^{\prime} \leq j-2$ that appear in the 2-parameter solution $\lambda(t, \eta ; \alpha, \beta)$ of $\left(P_{J}\right)$. Thus $z_{J}(x, t, \eta)$ and $s_{J}(t, \eta)$ have alternating parity.

(vii) The following equality holds:

$$
\begin{aligned}
Q_{J}(x, t, \eta)= & \left(\frac{\partial z_{J}(x, t, \eta)}{\partial x}\right)^{2} Q_{\mathrm{can}}\left(z_{J}(x, t, \eta), s_{J}(t, \eta), \eta ; A_{J}(\eta), B_{J}(\eta)\right) \\
& -\frac{1}{2} \eta^{-2}\left\{z_{J}(x, t, \eta) ; x\right\}
\end{aligned}
$$


where $\left\{z_{J}(x, t, \eta) ; x\right\}$ denotes the Schwarzian derivative,

$$
\begin{aligned}
& \left\{z_{J}(x, t, \eta) ; x\right\} \\
& \quad=\left(\frac{\partial^{3} z_{J}(x, t, \eta)}{\partial x^{3}} / \frac{\partial z_{J}(x, t, \eta)}{\partial x}\right)-\frac{3}{2}\left(\frac{\partial^{2} z_{J}(x, t, \eta)}{\partial x^{2}} / \frac{\partial z_{J}(x, t, \eta)}{\partial x}\right)^{2} .
\end{aligned}
$$

The proof of [KT2] also tells us that the formal series appearing in Theorem 2.7 are determined by the following process. First, the formal series $z_{J}(x, t, \eta)$ is fixed by [AKT2, Theorem 3.1]. In particular, the top term $z_{0}(x, t)$ is given with a suitable choice of the square root as follows:

$$
z_{0}(x, t)=\left[\int_{\lambda_{0}(t)}^{x} \sqrt{Q_{J, 0}(x, t)} d x\right]^{1 / 2} .
$$

Next, in view of (2.36), we find formal power series $A_{J}(\eta)$ and $B_{J}(\eta)$ (not unique) which satisfy

$$
-16 A_{J}(\eta) B_{J}(\eta)=E_{J}(\eta)
$$

Fixing $\left(A_{J}(\eta), B_{J}(\eta)\right)$, we can find a formal series $s_{J}(t, \eta)$ so that

$$
\sigma\left(s_{J}(t, \eta), \eta ; A_{J}(\eta), B_{J}(\eta)\right)=\eta^{1 / 2} z_{J}\left(\lambda_{J}(t, \eta ; \alpha, \beta), t, \eta\right) .
$$

Here $\lambda_{J}$ is the 2-parameter solution of $\left(P_{J}\right)$ substituted into the coefficients of $\left(S L_{J}\right)$ and $\left(D_{J}\right)$. The top term $s_{0}(t)$ in $s_{J}(t, \eta)$ is given by

$$
s_{0}(t)=\frac{1}{2} \phi_{J}(t)=\frac{1}{2} \int^{t} \sqrt{F_{J}^{(1)}(t)} d t .
$$

Then the set of formal series $\left(z_{J}(x, t, \eta), s_{J}(t, \eta), A_{J}(\eta), B_{J}(\eta)\right)$ satisfies the conditions in Theorem 2.7. Note that there is an ambiguity in the above choice of formal series; if a set of formal series

$$
\left(z_{J}(x, t, \eta), s_{J}(t, \eta), A_{J}(\eta), B_{J}(\eta)\right)
$$

satisfies the conditions in Theorem 2.7, then so does

$$
\left(z_{J}(x, t, \eta), s_{J}(t, \eta)+G(\eta), A_{J}(\eta) \exp (-2 \eta G(\eta)), B_{J}(\eta) \exp (2 \eta G(\eta))\right) .
$$

Here

$$
G(\eta)=\sum_{n=1}^{\infty} \eta^{-n} G_{n}
$$

is an arbitrary formal power series with constant coefficients $G_{n}$. Here we have assumed that the formal power series (2.47) has no constant term $G_{0}$. If we allow 
the constant term $G_{0} \neq 0$, then $A_{J}(\eta) \exp (-2 \eta G(\eta))$ is no longer a formal power series in $\eta^{-1}$, and hence we set $G_{0}=0$. The existence of this ambiguity corresponds to the fact that the relation between the parameters $(\alpha, \beta)$ and $(A, B)$ is given by essentially one relation, i.e., (2.43).

We will regard the coefficients $G_{n}$ in $(2.47)$ as free parameters. As is clear from (2.46), such free parameters are contained in the transformation series $s_{J}(t, \eta)$ additively, and it is shown in [KT2, Proposition 3.2] that the formal series $s_{J}(t, \eta)$ is unique up to these additive free parameters (see [KT2, Remark 3.3]). Once the free parameters $G_{n}$ are fixed, the transformation from $\left(S L_{J}\right)$ and $\left(D_{J}\right)$ to (Can) and $D_{\text {can }}$ is fixed, and hence the correspondence between the solutions of $\left(H_{J}\right)$ and $\left(H_{\text {can }}\right)$ is also fixed. These free parameters will be fixed when we discuss the transformation theory between Painlevé transcendents in Sections 4 and 5.

\section{§3. Stokes geometries of Painlevé equations and isomonodromy systems}

In [KT1], $[\mathrm{KT} 2]$ etc. the relationship between $P$-turning points, $P$-Stokes curves of $\left(P_{J}\right)$ and turning points, Stokes curves of $\left(S L_{J}\right)$ plays an important role in the construction of WKB theoretic transformations. In this section we review these geometric properties of Stokes geometries of $\left(P_{J}\right)$ and $\left(S L_{J}\right)$.

\section{$\S 3.1 . P$-Stokes geometry of $\left(P_{J}\right)$}

First, we review the definition of $P$-turning points and $P$-Stokes curves of $\left(P_{J}\right)$ introduced by Kawai and Takei. Here we recall that $\operatorname{Sing}_{J}$ is the set of singular points of $\left(P_{J}\right)$ defined in $(2.1)$.

Definition 3.1 ([KT1, Definition 2.1]). Let $\lambda_{J}=\lambda_{J}(t, \eta ; \alpha, \beta)$ be a 2 -parameter solution of $\left(P_{J}\right)$ and $\lambda_{0}(t)$ be its top term.

- A point $t=r \notin \operatorname{Sing}_{J}$ is said to be a P-turning point of $\lambda_{J}$ if

$$
F_{J}^{(1)}(r)=0
$$

where $F_{J}^{(1)}(t)$ is defined by $(2.7)$.

- A $P$-turning point $t=r$ of $\lambda_{J}$ is called simple if

$$
\frac{\partial^{2} F_{J}}{\partial \lambda^{2}}\left(\lambda_{0}(r), r\right) \neq 0 \text {. }
$$

- For a $P$-turning point $t=r$ of $\lambda_{J}$, a P-Stokes curve of $\lambda_{J}$ (emanating from $t=r)$ is an integral curve defined by

$$
\operatorname{Im} \int_{r}^{t} \sqrt{F_{J}^{(1)}(t)} d t=0 .
$$


$P$-turning points and $P$-Stokes curves of $\lambda_{J}$ are defined only in terms of the top term $\lambda_{0}(t)$ of the 2-parameter solution in question. Although they are defined for a fixed branch of the algebraic function $\lambda_{0}(t)$, we may regard them as objects on the Riemann surface of $\lambda_{0}(t)$. By "a $P$-turning point (resp., a $P$-Stokes curve)" we may simply mean "a $P$-turning point (resp., a $P$-Stokes curve) of some 2parameter solution $\lambda_{J}$ ". Note also that $P$-turning points and $P$-Stokes curves are nothing but zeros and horizontal trajectories (see [St]) of the quadratic differential $F^{(1)}(t) d t^{2}$ defined on the Riemann surface of $\lambda_{0}(t)$.

As is pointed out in [WT] and [T4], a point $s \in \operatorname{Sing}_{J}$ contained in the following list may play a role similar to $P$-turning points:

- $s=0$ for $\left(P_{\mathrm{III}^{\prime}\left(D_{6}\right)}\right),\left(P_{\mathrm{III}^{\prime}\left(D_{7}\right)}\right),\left(P_{\mathrm{III}^{\prime}\left(D_{8}\right)}\right),\left(P_{\mathrm{V}}\right)$ and $\left(P_{\mathrm{VI}}\right)$,

- $s=1$ for $\left(P_{\mathrm{VI}}\right)$,

- $s=\infty$ for $\left(P_{\mathrm{VI}}\right)$.

At a singular point $s$ in the above list, there exists a simple-pole type 2-parameter solution, that is, the top term $\lambda_{0}(t)$ of a 2-parameter solution has a branch point at $t=s$ satisfying

$$
F_{J}^{(1)}(t)=O\left((t-s)^{-3 / 2}\right) \quad \text { as } t \rightarrow s
$$

Note that (3.4) guarantees that the corresponding quadratic differential $F^{(1)}(t) d t^{2}$ has a simple-pole type singularity at $t=s$ after taking a new independent variable $T=(t-s)^{1 / 2}$, which is a local parameter of the Riemann surface of $\lambda_{0}(t)$ near $t=s$. On the Riemann surface of $\lambda_{0}(t)$ we distinguish such singular points from usual singular points, and call them P-turning points of simple-pole type. A Pturning point of simple-pole type is denoted by $r_{\mathrm{sp}}$. A $P$-Stokes curve emanating from $r_{\mathrm{sp}}$ is also defined by

$$
\operatorname{Im} \int_{r_{\mathrm{sp}}}^{t} \sqrt{F_{J}^{(1)}(t)} d t=0 .
$$

By the $P$-Stokes geometry (of $\left(P_{J}\right)$ ) we mean the configuration of $P$-turning points, $P$-turning points of simple-pole type, singular points and $P$-Stokes curves (of $\left(P_{J}\right)$ ). Figure 2 depicts examples of $P$-Stokes geometries. Five $P$-Stokes curves emanate from each simple $P$-turning point. Figure $2(\mathrm{~b})$ shows an example of $P_{\mathrm{III}^{\prime}\left(D_{6}\right)}$ which has a $P$-turning point of simple-pole type at the origin, and one $P$ Stokes curve emanates from the $P$-turning point of simple-pole type. Since $\lambda_{0}(t)$ is a multi-valued function of $t, P$-Stokes curves intersect each other, as can be seen in the figures. Such "apparent" intersections are resolved if we take a lift of $P$-Stokes curves onto the Riemann surface of $\lambda_{0}(t)$ (see Section 3.3 below). 


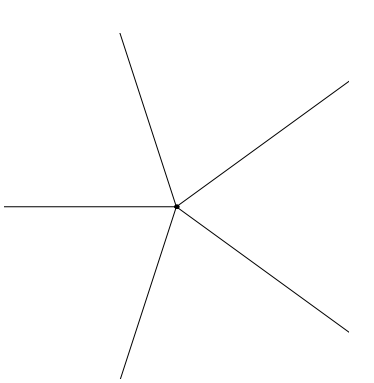

(a): $\left(P_{\mathrm{I}}\right)$

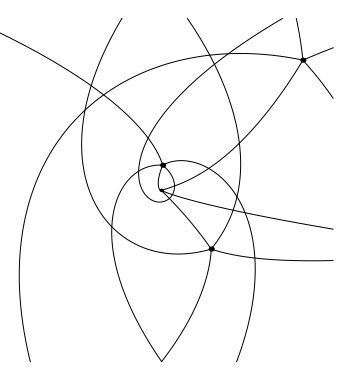

(b): $\left(P_{\mathrm{III}^{\prime}\left(D_{6}\right)}\right)$ with

$\left(c_{0}, c_{\infty}\right)=(3,2+i)$

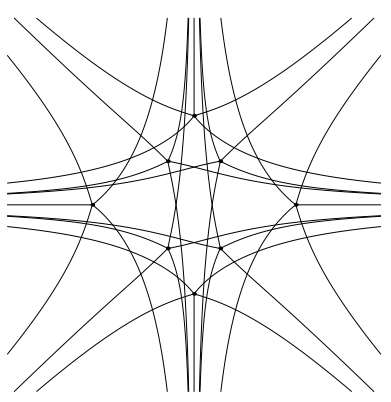

(c): $\left(P_{\mathrm{IV}}\right)$ with $\left(c_{0}, c_{\infty}\right)=(1,2)$

Figure 2. Examples of $P$-Stokes geometries.

Remark 3.2. P-Stokes curves are used to describe the criterion of Borel summability of 0-parameter solutions (i.e., formal power series solutions of the form $\left.\lambda(t, \eta)=\sum_{n=0}^{\infty} \eta^{-n} \lambda_{n}(t)\right)$ of $\left(P_{J}\right)$ by [KaKo]. It is known that certain non-linear Stokes phenomena occur for such a formal solution of $\left(P_{J}\right)$ on $P$-Stokes curves. Takei discussed such Stokes phenomena for $\left(P_{\mathrm{I}}\right)$ in [T1]. Moreover, it is also expected that non-linear Stokes phenomena also occur for 2-parameter solutions (see [T2]).

\section{§3.2. Stokes geometry of $\left(S L_{J}\right)$}

Next, we recall the definition of turning points and Stokes curves for the linear differential equation $\left(S L_{J}\right)$, and explain their relationship to the $P$-Stokes geometry defined in the previous subsection. Recall that we consider the situation that a 2-parameter solution $\left(\lambda_{J}, \nu_{J}\right)=\left(\lambda_{J}(t, \eta ; \alpha, \beta), \nu_{J}(t, \eta ; \alpha, \beta)\right)$ of $\left(H_{J}\right)$ is substituted into $(\lambda, \nu)$ which appears in the coefficients of $\left(S L_{J}\right)$ and $\left(D_{J}\right)$, as explained in Section 2.2. Here we assume that the 2-parameter solution is defined in a neighborhood $V$ of a point $t_{*} \in \Omega_{J}$, and the branch of $\lambda_{0}(t)$, which is the top term of $\lambda_{J}$, is fixed on $V$.

Definition 3.3 ([KT3, Definitions 2.4 and 2.6]). Fix a point $t$ contained in $V$.

- A point $x=a(t)$ is called a turning point of $\left(S L_{J}\right)$ (at $t$ ) if it is a zero of $Q_{J, 0}(x, t)$.

- A Stokes curve of $\left(S L_{J}\right)$ is an integral curve emanating from a turning point $x=a(t)$ defined by

$$
\operatorname{Im} \int_{a(t)}^{x} \sqrt{Q_{J, 0}(x, t)} d x=0 .
$$

Remark 3.4. Note that location of turning points and Stokes curves for $\left(S L_{J}\right)$ depends on $t$. More precisely, it also depends on the branch of $\lambda_{0}$ at $t$, which is the 
top term of the 2-parameter solution substituted. Therefore, by "turning points (resp., Stokes curves) of $\left(S L_{J}\right)$ at $t \in V$ " we mean "turning points (resp., Stokes curves) of $\left(S L_{J}\right)$ at $t$ with the fixed branch of $\lambda_{0}$ on $V$ ".

Turning points and Stokes curves of $\left(S L_{J}\right)$ are nothing but zeros and horizontal trajectories of the quadratic differential $Q_{J, 0}(x, t) d x^{2}$. We say that a turning point is of order $m$ if it is a zero of $Q_{J, 0}$ of order $m$. In particular, turning points of order 1 and 2 are called simple and double turning points, respectively. In view of (2.14), in a generic situation $\left(S L_{J}\right)$ has a double turning point at $x=\lambda_{0}(t)$ and one simple turning point (resp., two simple turning points) when $J=\mathrm{I}, \operatorname{III}^{\prime}\left(D_{7}\right)$ and $\operatorname{III}^{\prime}\left(D_{8}\right)$ (resp., $J=\mathrm{II}, \mathrm{III}^{\prime}\left(D_{6}\right), \mathrm{IV}, \mathrm{V}$ and VI). In the case of a linear equation, $m+2$ Stokes curves emanate from a turning point of order $m(m \geq 1)$. By the Stokes geometry (of $\left(S L_{J}\right)$ ) we mean the configuration of turning points, singular points and Stokes curves (for a fixed $t$ ). Actually, if $Q_{J, 0}(x, t)$ has simple poles, we need to regard them as turning points similarly to $P$-turning points of simple-pole type of $\left(P_{J}\right)$ (see $\left.[\mathrm{Ko}]\right)$. However, in view of (2.14), such a simple pole does not appear in a generic situation, and we will only consider situations where a simple pole never appears in the Stokes geometry of $\left(S L_{J}\right)$.

Figure 3 depicts examples of Stokes curves of $\left(S L_{\mathrm{I}}\right)$ for several $t$. Here $t_{1}$ and $t_{3}$ are some points which do not lie on a $P$-Stokes curve of $\left(P_{\mathrm{I}}\right)$, while $t_{2}$ lies on a

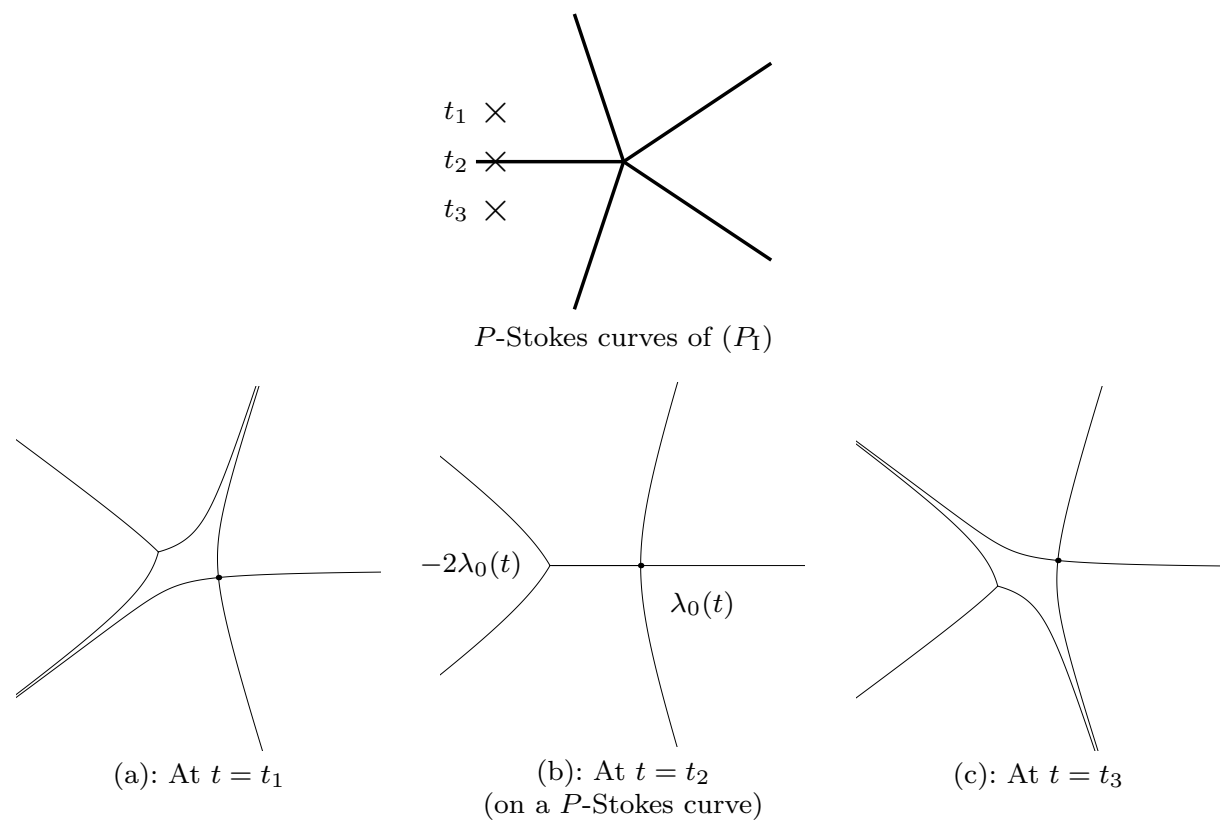

Figure 3. Stokes curves of $\left(S L_{\mathrm{I}}\right)$ (for several $t$ ). 
$P$-Stokes curve $\left(P_{\mathrm{I}}\right) .\left(S L_{\mathrm{I}}\right)$ has a double turning point at $x=\lambda_{0}(t)$ and a simple turning point at $x=-2 \lambda_{0}(t)$ when $t \neq 0$. Note that since $\lambda_{0}(t)=\sqrt{-t / 6}$ for $\left(P_{\mathrm{I}}\right)$, these two turning points merge as $t$ tends to the $P$-turning point $t=0$. We can observe that a Stokes curve of $\left(S L_{\mathrm{I}}\right)$ connects the two turning points $x=\lambda_{0}(t)$ and $-2 \lambda_{0}(t)$ when $t=t_{2}$ which lies on a $P$-Stokes curve. We call such a Stokes curve connecting turning points of $\left(S L_{J}\right)$ a degenerate Stokes segment, or a Stokes segment for short. (In the context of quadratic differentials Stokes segments are called saddle connections.)

Actually, other $\left(P_{J}\right)$ and $\left(S L_{J}\right)$ also enjoy the same geometric properties as $\left(P_{\mathrm{I}}\right)$ and $\left(S L_{\mathrm{I}}\right)$ explained here. That is, $P$-turning points and $P$-Stokes curves for $\left(P_{J}\right)$ are related to turning points and Stokes curves for $\left(S L_{J}\right)$ in the following manner.

Proposition 3.5 ([KT1, Proposition 2.1]). (i) For a simple P-turning point $r$ (of $\left.\lambda_{J}\right)$, there exists a simple turning point $a(t)$ of $\left(S L_{J}\right)$ that merges with the double turning point $x=\lambda_{0}(t)$ at $t=r$, and consequently at $t=r$ there exists a turning point of order three for $\left(S L_{J}\right)$.

(ii) For the simple P-turning point $r$ and the turning point a $(t)$ of $\left(S L_{J}\right)$ as above, the following equality holds:

$$
\int_{a(t)}^{\lambda_{0}(t)} \sqrt{Q_{J, 0}(x, t)} d x=\frac{1}{2} \int_{r}^{t} \sqrt{F_{J}^{(1)}(t)} d t .
$$

Here the branches of the square roots are chosen so that

$$
\sqrt{Q_{J, 0}(x, t)}=C_{J}(x, t)\left(x-\lambda_{0}\right) \sqrt{R_{J}(x, t)}, \quad \sqrt{R_{J}\left(\lambda_{0}(t), t\right)}=\sqrt{F_{J}^{(1)}(t)} .
$$

Proposition 3.5 implies that, when $t$ lies on a $P$-Stokes curve emanating from a simple $P$-turning point $r$, a Stokes segment appears between the double turning point $\lambda_{0}(t)$ and the simple turning point $a(t)$. This relationship between $P$-Stokes curves and Stokes curves is essential in the construction of WKB theoretic transformation to $\left(P_{\mathrm{I}}\right)$ near a simple $P$-turning point (see [KT1] and [KT2]).

Similar geometric properties are also observed when $t$ lies on a $P$-Stokes curve emanating from a $P$-turning point of simple-pole type.

Proposition 3.6 ([T4, Proposition 3.2(ii)]). Suppose that $t$ lies on a P-Stokes curve emanating from a P-turning point of $\left(P_{J}\right)$ of simple-pole type. Then there exists a Stokes curve of $\left(S L_{J}\right)$ which starts from $\lambda_{0}(t)$ and returns to $\lambda_{0}(t)$ after encircling several singular points and/or turning points of $\left(S L_{J}\right)$. 


\section{§3.3. Degeneration of P-Stokes geometry}

As is explained in the Introduction, we are interested in the degenerate situations of $P$-Stokes geometry, that is, situations where there exists a $P$-Stokes curve which connects $P$-turning points or $P$-turning points of simple-pole type of a 2 -parameter solution $\lambda_{J}$ of $\left(P_{J}\right)$. We will call such special $P$-Stokes curves degenerate $P$-Stokes segments, or P-Stokes segments for short. In this section we discuss a relationship between such a degeneration of the $P$-Stokes geometry of $\left(P_{J}\right)$ and the Stokes geometry of $\left(S L_{J}\right)$.

Typically, there are two types of $P$-Stokes segments which appear in a generic situation: A P-Stokes segment of the first type connects two different simple $P$-turning points, while a $P$-Stokes segment of the second type (sometimes called loop type) emanates from and returns to the same $P$-turning point and hence forms a closed loop.

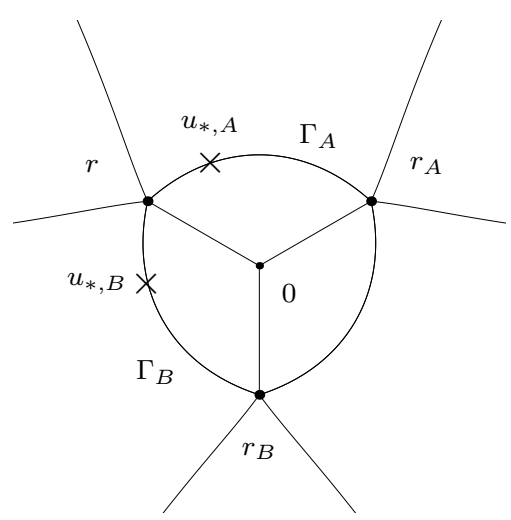

Figure 4. The $P$-Stokes geometry of $\left(P_{\mathrm{II}}\right)$ with $P$-Stokes segments (described on the $u$-plane).

Figure 4 depicts the $P$-Stokes geometry of $\left(P_{\mathrm{II}}\right)$ when $c=i$, and we can observe that three $P$-Stokes segments appear in the figure. Here we have introduced a new variable

$$
u=\lambda_{0}(t)
$$

of the Riemann surface of $\lambda_{0}(t)$, and Figure 4 describes the $P$-Stokes curves of $\left(P_{\mathrm{II}}\right)$ on the $u$-plane. Using the relation $t=-\left(2 u^{3}+c\right) / u$, the quadratic differential which defines the $P$-Stokes geometry of $\left(P_{\mathrm{II}}\right)$ can be written as

$$
F_{\text {II }}^{(1)}(t) d t^{2}=\operatorname{quad}_{\mathrm{II}}(u, c) d u^{2}, \quad \operatorname{quad}_{\mathrm{II}}(u, c)=\left(4 u^{3}-c\right)^{3} / u^{5},
$$


in the $u$-variable. Although Figure 4 depicts the case $c=i$, the $P$-Stokes geometry of $\left(P_{\mathrm{II}}\right)$ described in the variable $u$ given in (3.9) for any $c \in i \mathbb{R}_{>0}$ is the same as in Figure 4 since the quadratic differential (3.10) has the following scale invariance:

$$
r^{-1} \sqrt{\operatorname{quad}_{\mathrm{II}}\left(r^{1 / 3} u, r c\right)} d\left(r^{1 / 3} u\right)=\sqrt{\operatorname{quad}_{\mathrm{II}}(u, c)} d u \quad(r \neq 0) .
$$

Therefore, when $c \in i \mathbb{R}_{>0}$, the $P$-Stokes geometry of $\left(P_{\mathrm{II}}\right)$ has three simple $P$-turning points and three $P$-Stokes segments. The symbols $r, r_{A}$ and $r_{B}$ (resp., $\left.\Gamma_{A}, \Gamma_{B}\right)$ in Figure 4 represent the $P$-turning points (resp., $P$-Stokes segments)

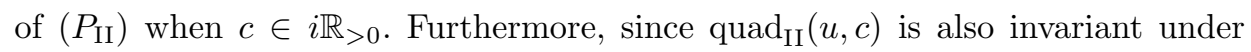
$(u, c) \mapsto(-u,-c)$, the $P$-Stokes geometry when $c \in i \mathbb{R}_{<0}$ is the reflection $u \mapsto-u$ of Figure 4.

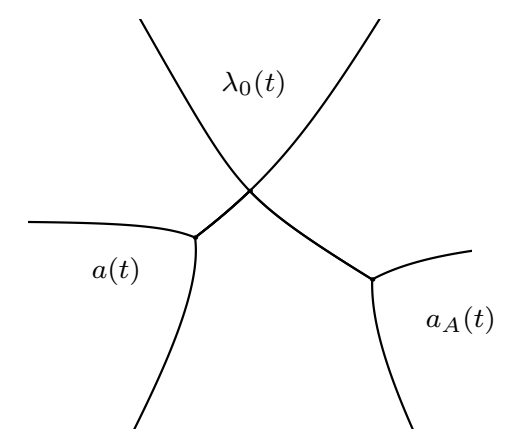

$\left(S L_{\mathrm{II}}-A\right):$ The Stokes geometry of $\left(S L_{\mathrm{II}}\right)$ corresponding to $u_{*, A}$

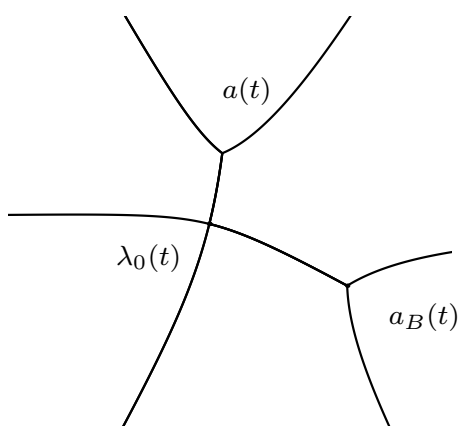

$\left(S L_{\mathrm{II}}-B\right)$ : The Stokes geometry of $\left(S L_{\mathrm{II}}\right)$ corresponding to $u_{*, B}$

Figure 5. The Stokes geometries of $\left(S L_{\mathrm{II}}\right)$ on $P$-Stokes segments.

Figure $5\left(S L_{\mathrm{II}}-A\right)$ (resp., $\left.\left(S L_{\mathrm{II}}-B\right)\right)$ depicts the Stokes geometry of $\left(S L_{\mathrm{II}}\right)$ when we fix $t$ at a point $t_{*, A}$ (resp., $t_{*, B}$ ) corresponding to a point $u_{*, A}$ (resp., $\left.u_{*, B}\right)$ which lies on the $P$-Stokes segment $\Gamma_{A}$ (resp., $\Gamma_{B}$ ) in Figure 4. Note that $u$ determines a point $t$ on the $t$-plane together with a branch of $\lambda_{0}$ at $t$, and the Stokes geometries shown in Figure 5 are drawn for the branch of $\lambda_{0}$ determined by $u_{*, A}$ and $u_{*, B}$, respectively (see Remark 3.4). In both cases of Figure 5, there are two Stokes segments in the Stokes geometry of $\left(S L_{\mathrm{II}}\right)$, each connecting the double turning point $x=\lambda_{0}(t)$ and a simple turning point. Here $a(t), a_{A}(t)$ and $a_{B}(t)$ are the simple turning points of $\left(S L_{\mathrm{II}}\right)$ which merge with $\lambda_{0}(t)$ at the $P$-turning points $r, r_{A}$ and $r_{B}$, respectively (cf. Proposition 3.5(i)). The points $a_{A}(t)$ and $a_{B}(t)$ merge with $\lambda_{0}(t)$ when $t$ tends to $r_{A}$ and $r_{B}$ along the $P$-Stokes segment $\Gamma_{A}$ or $\Gamma_{B}$, respectively. 


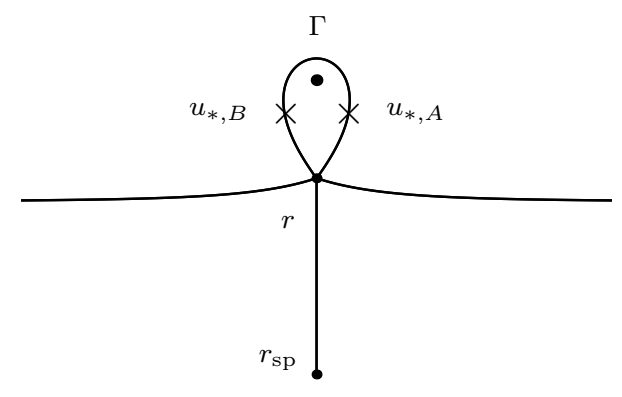

Figure 6 . The P-Stokes geometry of $\left(P_{\mathrm{III}^{\prime}\left(D_{7}\right)}\right)$ with a loop-type $P$-Stokes segment (described on the $u$-plane).

On the other hand, Figure 6 depicts the $P$-Stokes geometry of $\left(P_{\mathrm{III}^{\prime}\left(D_{7}\right)}\right)$ when $c=i$ by using a new variable

$$
u=\frac{2 \lambda_{0}(t)^{2}}{c \lambda_{0}(t)-t}
$$

of the Riemann surface of $\lambda_{0}(t)$. Since $t=-u^{2}(u-c) / 2$, the quadratic differential becomes

$$
\begin{aligned}
& F_{\mathrm{III}^{\prime}\left(D_{7}\right)}^{(1)}(t) d t^{2}=\operatorname{quad}_{\mathrm{III}^{\prime}\left(D_{7}\right)}(u, c) d u^{2}, \\
& \operatorname{quad}_{\mathrm{III}\left(D_{7}\right)}(u, c)=\frac{(3 u-2 c)^{3}}{u(u-c)^{2}} d u^{2} .
\end{aligned}
$$

Hence there is one simple $P$-turning point and one $P$-turning point of simplepole type in the $P$-Stokes geometry of $\left(P_{\mathrm{III}^{\prime}\left(D_{7}\right)}\right)$. In Figure 6 we can observe that a $P$-Stokes segment of loop type, denoted by $\Gamma$, appears around the double pole $u=c$ of (3.12). It is known that such a loop occurs when the residue of $\sqrt{\text { quad }_{\mathrm{III}^{\prime}\left(D_{7}\right)}(u, c)} d u$ at $u=c$ is purely imaginary (see [St, Section 7]). Since the quadratic differential (3.12) satisfies

$$
r^{-1} \sqrt{\operatorname{quad}_{\mathrm{III}^{\prime}\left(D_{7}\right)}(r u, r c)} d(r u)=\sqrt{\operatorname{quad}_{\mathrm{III}\left(D_{7}\right)}(u, c)} d u
$$

for any $r \neq 0$, we conclude that the $P$-Stokes geometry of $\left(P_{\mathrm{III}^{\prime}\left(D_{7}\right)}\right)$ (described in the variable $u$ given by (3.11)) when $c \in i \mathbb{R}_{>0}$ is the same as in Figure 6 . Furthermore, since quad $\operatorname{III}^{\prime}\left(D_{7}\right)(u, c)$ is also invariant under $(u, c) \mapsto(-u,-c)$, the $P$-Stokes geometry when $c \in i \mathbb{R}_{<0}$ is the reflection $u \mapsto-u$ of Figure 6 .

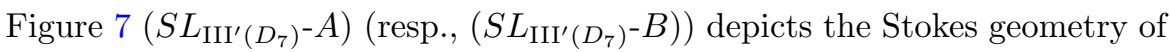
$\left(S L_{\mathrm{III}^{\prime}\left(D_{7}\right)}\right)$ when $t$ is a point $t_{*, A}$ (resp., $\left.t_{*, B}\right)$ corresponding to $u_{*, A}$ (resp., $u_{*, B}$ ) which lies on the loop-type $P$-Stokes segment $\Gamma$ in Figure 6 . There are two Stokes 


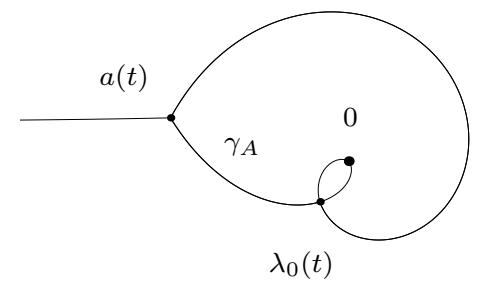

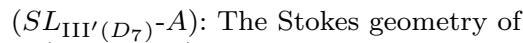
$\left(S L_{\mathrm{III}^{\prime}\left(D_{7}\right)}\right)$ corresponding to $u_{*, A}$

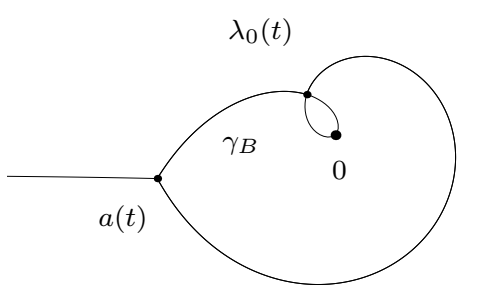

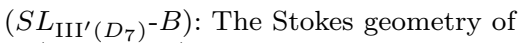
$\left(S L_{\mathrm{III}^{\prime}\left(D_{7}\right)}\right)$ corresponding to $u_{*, B}$

Figure 7. The Stokes geometries of $\left(S L_{\mathrm{III}^{\prime}\left(D_{7}\right)}\right)$ on the loop-type $P$-Stokes segment.

segments in the Stokes geometry of $\left(S L_{\mathrm{III}}\left(D_{7}\right)\right)$, both connecting the double turning point $\lambda_{0}(t)$ and the same simple turning point $a(t)$. When $t$ tends to the simple $P$-turning point $r$ along $\Gamma$ in Figure 6, one of the two Stokes segments shrinks to a point (cf. Proposition 3.5(i)). In Figure $7\left(S L_{\mathrm{III}^{\prime}\left(D_{7}\right)}\right.$ - $\left.A\right)$ (resp., $\left.\left(S L_{\mathrm{III}^{\prime}\left(D_{7}\right)^{-}}-B\right)\right)$ the Stokes segment $\gamma_{A}$ (resp., $\gamma_{B}$ ) shrinks to a point when $t$ tends to $r$ along $\Gamma$ in the clockwise (resp., counter-clockwise) direction.

In Figures 5 and 7 we can observe common properties of the Stokes geometries of $\left(S L_{J}\right)$ 's when $t$ lies on a $P$-Stokes segment. Firstly, there appear two Stokes segments each of which connects the double turning point $\lambda_{0}(t)$ and a simple turning point. Secondly, these two Stokes segments are adjacent in the Stokes curves emanating from $\lambda_{0}(t)$. We can show that these properties hold for the Stokes geometry of $\left(S L_{J}\right)$ when $t$ lies on a $P$-Stokes segment of $\left(P_{J}\right)$.

Proposition 3.7. Let $r_{1}$ and $r_{2}$ be (possibly the same) simple P-turning points of $\lambda_{J}$ which are not of simple-pole type, and $a_{1}(t)$ and $a_{2}(t)$ be the simple turning points of $\left(S L_{J}\right)$ corresponding to $r_{1}$ and $r_{2}$ by Proposition 3.5(i). Suppose that $r_{1}$ and $r_{2}$ are connected by a $P$-Stokes segment $\Gamma$, and take a point $t_{*}$ which lies on $\Gamma$ as in Figure 8. Then there are two Stokes segments $\gamma_{1}$ and $\gamma_{2}$ in the Stokes geometry of $\left(S L_{J}\right)$ when $t=t_{*}$, where $\gamma_{1}$ (resp., $\left.\gamma_{2}\right)$ connects $\lambda_{0}\left(t_{*}\right)$ and $a_{1}\left(t_{*}\right)$ $\left(\right.$ resp., $\left.a_{2}\left(t_{*}\right)\right)$. Moreover, $\gamma_{1}$ and $\gamma_{2}$ are adjacent Stokes curves in the four Stokes curves emanating from $x=\lambda_{0}\left(t_{*}\right)$.

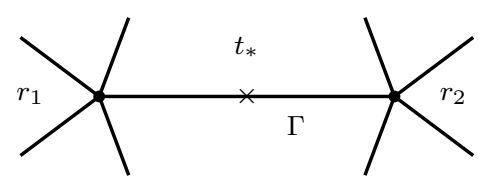

Figure 8. A $P$-Stokes segment $\Gamma$ and two simple $P$-turning points $r_{1}$ and $r_{2}$. 
Proof. Since $t_{*}$ lies on P-Stokes curves emanating from $r_{1}$ and $r_{2}$ simultaneously, it follows from Proposition 3.5 that the double turning point $x=\lambda_{0}(t)$ lies on both Stokes curves emanating from $a_{1}(t)$ and $a_{2}(t)$ when $t=t_{*}$. Hence, in the Stokes geometry of $\left(S L_{J}\right)$ there are two Stokes segments $\gamma_{1}$ and $\gamma_{2}$ which connect $\lambda_{0}\left(t_{*}\right)$ and $a_{1}\left(t_{*}\right)$ and $a_{2}\left(t_{*}\right)$, respectively. Thus in Figure 9 two cases can occur: In case (i) (resp., (ii)) $\gamma_{1}$ and $\gamma_{2}$ are adjacent (resp., opposite) Stokes curves which emanate from $\lambda_{0}$. However, case (ii) does not happen under our assumption, for the following reason.

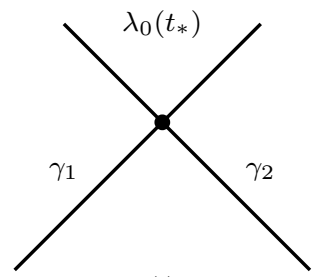

(i)

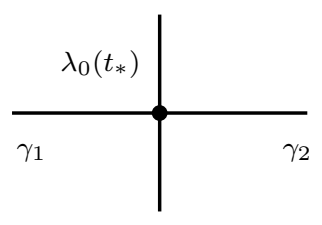

(ii)

Figure 9. Two candidates for Stokes segments.

For $k=1,2$, set

$$
\begin{aligned}
& \phi_{J, k}(t)=\int_{r_{k}}^{t} \sqrt{F_{J}^{(1)}(t)} d t, \\
& v_{J, k}(t)=\int_{a_{k}(t)}^{\lambda_{0}(t)} \sqrt{Q_{J, 0}(x, t)} d x .
\end{aligned}
$$

Proposition 3.5(ii) implies that $v_{J, k}(t)=\phi_{J, k}(t) / 2(k=1,2)$. The real parts of $\phi_{J, 1}\left(t_{*}\right)$ and $\phi_{J, 2}\left(t_{*}\right)$ have different signs since the real parts are increasing or decreasing along $P$-Stokes curves. Thus the real parts of $v_{J, 1}\left(t_{*}\right)$ and $v_{J, 2}\left(t_{*}\right)$ also have different signs. Therefore, case (ii) in Figure 9 never happens and only case (i) appears.

Proposition 3.7 yields the following two possibilities for the geometric type of the Stokes geometry of $\left(S L_{J}\right)$ when $t_{*}$ lies on a P-Stokes segment (cf. Figure 10):

(a) The double turning point $\lambda_{0}\left(t_{*}\right)$ is connected with two different simple turning points by two Stokes segments. This case is shown in Figure 5.

(b) The double turning point $\lambda_{0}\left(t_{*}\right)$ is connected with the same simple turning point by two Stokes segments. This case appears in Figure 7.

The following fact will be used in the proof of our main results. 


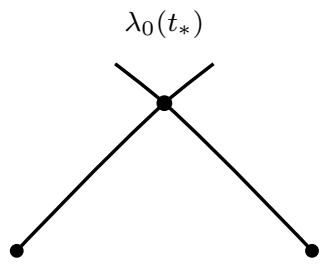

(a)

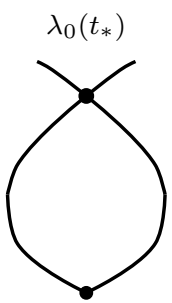

(b)

Figure 10. Two Stokes segments in the Stokes geometry of $\left(S L_{J}\right)$.

Lemma 3.8. In the situation of Proposition 3.7, we have

$$
\int_{a_{1}(t)}^{a_{2}(t)} \sqrt{Q_{J, 0}(x, t)} d x=\frac{1}{2} \int_{r_{1}}^{r_{2}} \sqrt{F_{J}^{(1)}(t)} d t .
$$

Here the integration path on the left-hand side is taken along a composition of two Stokes segments $\gamma_{1}$ and $\gamma_{2}$ in the Stokes geometry of $\left(S L_{J}\right)$, while that on the right-hand side is taken along the P-Stokes segment $\Gamma$.

Proof. Let $\phi_{J, k}(t)$ and $v_{J, k}(t)$ be functions defined in (3.13) and (3.14). Since $v_{J, k}(t)=\phi_{J, k}(t) / 2$ for $k=1,2$, we have $v_{J, 1}(t)-v_{J, 2}(t)=\left(\phi_{J, 1}(t)-\phi_{J, 2}(t)\right) / 2$. This gives the desired relation.

Lemma 3.8 entails that the integral of $\sqrt{Q_{J, 0}(x, t)} d x$ appearing (3.15) does not depend on $t$. Generally, the integral of $S_{J, \text { odd }}(x, t, \eta)$ along a closed cycle on the Riemann surface of $\sqrt{Q_{J, 0}(x, t)}$ is independent of $t$ by (2.22). In particular, from (3.15) and Table 6 we will deduce the following.

Lemma 3.9. (i) For $J=$ II, we have

$$
\int_{r_{1}}^{r_{2}} \sqrt{F_{\mathrm{II}}^{(1)}(t)} d t= \pm 2 \pi i c
$$

when $c \in i \mathbb{R}_{\neq 0}$. Here $r_{1}$ and $r_{2}$ are two simple P-turning points of $\left(P_{\mathrm{II}}\right)$ connected by a P-Stokes segment, and the integration path is taken along the $P$-Stokes segment. The sign \pm depends on the branch of the square root.

(ii) For $J=\operatorname{III}^{\prime}\left(D_{7}\right)$, we have

$$
\int_{\Gamma} \sqrt{F_{\mathrm{III}^{\prime}\left(D_{7}\right)}^{(1)}(t)} d t= \pm 2 \pi i c
$$

when $c \in i \mathbb{R}_{\neq 0}$. Here the integration path is taken along the loop-type P-Stokes segment $\Gamma$ of Figure 6 . The sign \pm depends on the branch of the square root. 


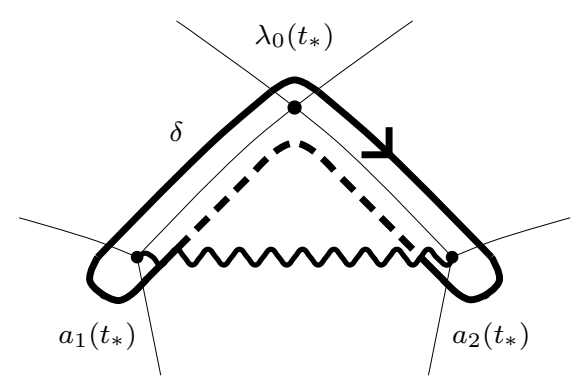

Figure 11. The cycle $\delta$.

Proof. We prove (3.16). Let $t_{*}$ be a point on the $P$-Stokes segment connecting $r_{1}$ and $r_{2}$, and $a_{1}(t)$ and $a_{2}(t)$ be the simple turning points of $\left(S L_{\mathrm{II}}\right)$ which correspond to $r_{1}$ and $r_{2}$ by Proposition 3.5(i). Then

$$
\int_{r_{1}}^{r_{2}} \sqrt{F_{\mathrm{II}}^{(1)}(t)} d t=2 \int_{a_{1}\left(t_{*}\right)}^{a_{2}\left(t_{*}\right)} \sqrt{Q_{\mathrm{II}, 0}\left(x, t_{*}\right)} d x
$$

by (3.15). The integral of $\sqrt{Q_{\mathrm{II}, 0}\left(x, t_{*}\right)} d x$ can be written as

$$
2 \int_{a_{1}\left(t_{*}\right)}^{a_{2}\left(t_{*}\right)} \sqrt{Q_{\mathrm{II}, 0}\left(x, t_{*}\right)} d x=\oint_{\delta} \sqrt{Q_{\mathrm{II}, 0}\left(x, t_{*}\right)} d x,
$$

where $\delta$ is a closed cycle on the Riemann surface of $\sqrt{Q_{\mathrm{II}, 0}\left(x, t_{*}\right)}$ described in Figure 11. The wiggly line in the figure represents the branch cut to determine the branch of $\sqrt{Q_{\mathrm{II}, 0}\left(x, t_{*}\right)}$, and the solid and dashed lines represent paths on the first and the second sheet of the Riemann surface of $\sqrt{Q_{\mathrm{II}, 0}\left(x, t_{*}\right)}$, respectively. Since the 1-form $\sqrt{Q_{\mathrm{II}, 0}\left(x, t_{*}\right)} d x$ has no singular point other than $x=a_{1}\left(t_{*}\right), a_{2}\left(t_{*}\right)$ and $\infty$, we have

$$
\oint_{\delta} \sqrt{Q_{\mathrm{II}, 0}\left(x, t_{*}\right)} d x=2 \pi i \operatorname{Res}_{x=\infty} \sqrt{Q_{\mathrm{II}, 0}\left(x, t_{*}\right)} d x= \pm 2 \pi i c .
$$

Here we have used (2.31) and Table 6 of residues. Thus we have proved (3.16). The equality (3.17) can be proved in the same manner by using the following fact:

$$
\operatorname{Res}_{x=0} \sqrt{Q_{\operatorname{III}\left(D_{7}\right), 0}(x, t)} d x= \pm c / 2 .
$$

\section{$\S 4$. WKB theoretic transformation to $\left(P_{\mathrm{II}}\right)$ on $P$-Stokes segments}

Here we show our main claims concerning WKB theoretic transformations between Painlevé transcendents on $P$-Stokes segments. Since we simultaneously deal with 
two different Painlevé equations $\left(P_{J}\right)$ and $\left(P_{\mathrm{II}}\right)$, in this section we put $\sim$ over variables or functions relevant to $\left(P_{J}\right)$ and $\left(S L_{J}\right)$ in order to avoid confusion.

\section{$\S 4.1$. Assumptions and statements}

Let $\left(\tilde{\lambda}_{J}, \tilde{\nu}_{J}\right)=\left(\tilde{\lambda}_{J}(\tilde{t}, \eta ; \tilde{\alpha}, \tilde{\beta}), \tilde{\nu}_{J}(\tilde{t}, \eta ; \tilde{\alpha}, \tilde{\beta})\right)$ be a 2-parameter solution of $\left(H_{J}\right)$ defined in a neighborhood of a point $\tilde{t}_{*} \in \Omega_{J}$, and consider $\left(S L_{J}\right)$ and $\left(D_{J}\right)$ with $\left(\tilde{\lambda}_{J}, \tilde{\nu}_{J}\right)$ substituted into their coefficients. Here we assume the following conditions.

Assumption 4.1. (1) $J \in\left\{\mathrm{II}, \mathrm{III}^{\prime}\left(D_{6}\right), \mathrm{IV}, \mathrm{V}, \mathrm{VI}\right\}$.

(2) There is a $P$-Stokes segment $\tilde{\Gamma}$ in the $P$-Stokes geometry of $\left(P_{J}\right)$ which connects two different simple $P$-turning points $\tilde{r}_{1}$ and $\tilde{r}_{2}$ of $\tilde{\lambda}_{J}$ (which are not of simple-pole type), and the point $\tilde{t}_{*}$ in question lies on $\tilde{\Gamma}$.

(3) The function (2.9) appearing in the instanton $\tilde{\Phi}_{J}(\tilde{t}, \eta)$ of the 2-parameter solution $\left(\tilde{\lambda}_{J}, \tilde{\nu}_{J}\right)$ is normalized at the simple $P$-turning point $\tilde{r}_{1}$ as

$$
\tilde{\phi}_{J}(\tilde{t})=\int_{\tilde{r}_{1}}^{\tilde{t}} \sqrt{\tilde{F}_{J}^{(1)}(\tilde{t})} d \tilde{t} .
$$

(4) The Stokes geometry of $\left(S L_{J}\right)$ at $\tilde{t}=\tilde{t}_{*}$ contains the same configuration as in Figure 10(a). That is, the double turning point $\tilde{\lambda}_{0}\left(\tilde{t}_{*}\right)$ is connected to two different simple turning points $\tilde{a}_{1}\left(\tilde{t}_{*}\right)$ and $\tilde{a}_{2}\left(\tilde{t}_{*}\right)$ by two Stokes segments $\tilde{\gamma}_{1}$ and $\tilde{\gamma}_{2}$, respectively. Here the labels of the simple turning points and the Stokes segments are assigned by the following rule: When $\tilde{t}$ tends to $\tilde{r}_{1}$ (resp., $\tilde{r}_{2}$ ) along $\tilde{\Gamma}, \tilde{a}_{1}(\tilde{t})$ (resp., $\left.\tilde{a}_{2}(\tilde{t})\right)$ merges with $\tilde{\lambda}_{0}(\tilde{t})$ (cf. Proposition 3.5).

(5) All singular points of $\tilde{Q}_{J, 0}\left(\tilde{x}, \tilde{t}_{*}\right.$ ) (as a function of $\tilde{x}$ ) are poles of even order.

Since the $P$-Stokes geometry for $J=\mathrm{I}, \operatorname{III}^{\prime}\left(D_{7}\right)$ and $\operatorname{III}^{\prime}\left(D_{8}\right)$ never contains a $P$-Stokes segment connecting two different simple $P$-turning points, we have excluded these cases. One of our main results below claims that under Assumption 4.1 we can construct a formal transformation series defined on a neighborhood of the union $\tilde{\gamma}_{1} \cup \tilde{\gamma}_{2}$ of two Stokes segments that brings $\left(S L_{J}\right)$ to $\left(S L_{\mathrm{II}}\right)$ with an appropriate 2-parameter solution $\left(\lambda_{\mathrm{II}}, \nu_{\mathrm{II}}\right)$ of $\left(H_{\mathrm{II}}\right)$ being substituted into $(\lambda, \nu)$ in $\left(S L_{\mathrm{II}}\right)$, in the following sense.

First, we fix the constant $c$ appearing in $\left(P_{\mathrm{II}}\right)$ and $\left(S L_{\mathrm{II}}\right)$ to be

$$
c=\frac{1}{2 \pi i} \int_{\tilde{r}_{1}}^{\tilde{r}_{2}} \sqrt{\tilde{F}_{J}^{(1)}(\tilde{t})} d \tilde{t},
$$

where the integration path is taken along the $P$-Stokes segment $\tilde{\Gamma}$. Since the function (4.1) is monotone and takes real values along $\tilde{\Gamma}$, the constant $c$ determined 


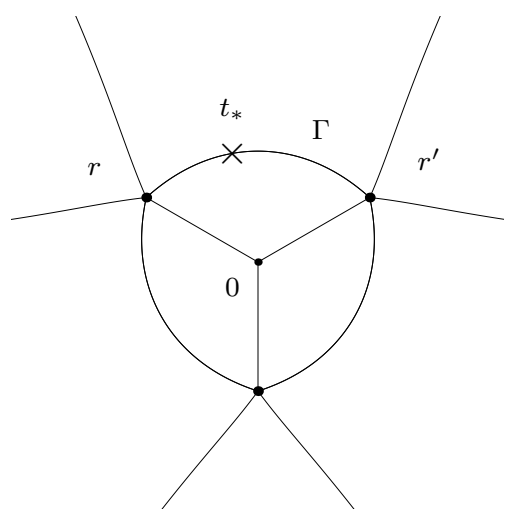

(P): P-Stokes geometry of $\left(P_{\mathrm{II}}\right)$ (described on the $u$-plane)

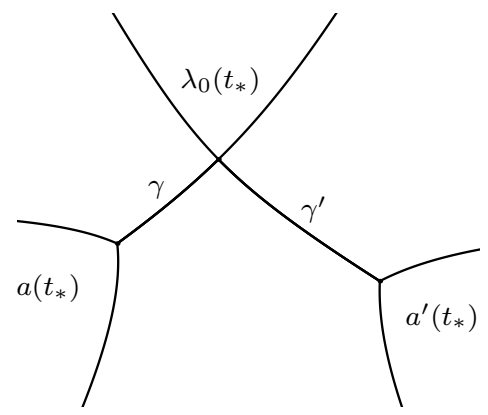

(SL): Stokes geometry of $\left(S L_{\mathrm{II}}\right)$ at $t=t_{*}$

Figure 12. The P-Stokes geometry of $\left(P_{\mathrm{II}}\right)$ and the Stokes geometry of $\left(S L_{\mathrm{II}}\right)$.

by (4.2) is non-zero and purely imaginary. Here we assume that $c \in i \mathbb{R}_{>0}$. Then the $P$-Stokes geometry of $\left(P_{\mathrm{II}}\right)$ (described in the variable $u$ given by $(3.9)$ ) when $c$ is given by $(4.2)$ is the same as in Figure $12(\mathrm{P})$. Thus, the $P$-Stokes geometry of $\left(P_{\mathrm{II}}\right)$ has three simple $P$-turning points, and three $P$-Stokes segments appear simultaneously. (As is remarked in Section 3.3, when $c \in i \mathbb{R}_{<0}$, the $P$-Stokes geometry of $\left(P_{\mathrm{II}}\right)$ is the reflection $u \mapsto-u$ of Figure $12(\mathrm{P})$. Our discussion below also applies to $c \in i \mathbb{R}_{<0}$.) Furthermore, we can verify that the corresponding Stokes geometry of $\left(S L_{\mathrm{II}}\right)$ on a $P$-Stokes segment is as in Figure $12(\mathrm{SL})$. That is, when we take any point $t_{*}$ on a $P$-Stokes segment of $\left(P_{\mathrm{II}}\right)$, say $\Gamma$ in Figure $12(\mathrm{P})$, then the corresponding Stokes geometry of $\left(S L_{\mathrm{II}}\right)$ has one double turning point at $x=\lambda_{0}\left(t_{*}\right)$ and two simple turning points $x=a\left(t_{*}\right)$ and $a^{\prime}\left(t_{*}\right)$, and there are two Stokes segments $\gamma$ and $\gamma^{\prime}$ which connect $\lambda_{0}\left(t_{*}\right)$ to these simple turning points. Note that $a(t)$ (resp., $a^{\prime}(t)$ ) merges with $\lambda_{0}(t)$ as $t$ tends to $r$ (resp., $r^{\prime}$ ) along $\Gamma$.

Having these geometric properties in mind, we formulate the precise statement of our first main result as follows.

Theorem 4.2. Under Assumption 4.1, for any 2-parameter solution $\left(\tilde{\lambda}_{J}, \tilde{\nu}_{J}\right)=$ $\left(\tilde{\lambda}_{J}(\tilde{t}, \eta ; \tilde{\alpha}, \tilde{\beta}), \tilde{\nu}_{J}(\tilde{t}, \eta ; \tilde{\alpha}, \tilde{\beta})\right)$ of $\left(H_{J}\right)$, there exist

- a domain $\tilde{U}$ which contains the union $\tilde{\gamma}_{1} \cup \tilde{\gamma}_{2}$ of two Stokes segments,

- a neighborhood $\tilde{V}$ of $\tilde{t}_{*}$,

- formal series

$$
x(\tilde{x}, \tilde{t}, \eta)=\sum_{j \geq 0} \eta^{-j / 2} x_{j / 2}(\tilde{x}, \tilde{t}, \eta), \quad t(\tilde{t}, \eta)=\sum_{j \geq 0} \eta^{-j / 2} t_{j / 2}(\tilde{t}, \eta)
$$


whose coefficients $\left\{x_{j / 2}(\tilde{x}, \tilde{t}, \eta)\right\}_{j=0}^{\infty}$ and $\left\{t_{j / 2}(\tilde{t}, \eta)\right\}_{j=0}^{\infty}$ are functions defined on $\tilde{U} \times \tilde{V}$ and $\tilde{V}$, respectively, and may depend on $\eta$,

- a 2-parameter solution

$$
\begin{aligned}
\left(\lambda_{\mathrm{II}}, \nu_{\mathrm{II}}\right) & =\left(\lambda_{\mathrm{II}}(t, \eta ; \alpha, \beta), \nu_{\mathrm{II}}(t, \eta ; \alpha, \beta)\right), \\
(\alpha, \beta) & =\left(\sum_{n=0}^{\infty} \eta^{-n} \alpha_{n}, \sum_{n=0}^{\infty} \eta^{-n} \beta_{n}\right)
\end{aligned}
$$

of $\left(H_{\mathrm{II}}\right)$ with the constant $c$ determined by (4.2), and the function (2.9) appearing in the instanton $\Phi_{\mathrm{II}}(t, \eta)$ that is normalized at a simple P-turning point $r_{1}$ of $\left(P_{\mathrm{II}}\right)$ as

$$
\phi_{\mathrm{II}}(t)=\int_{r_{1}}^{t} \sqrt{F_{\mathrm{II}}^{(1)}(t)} d t,
$$

which satisfy the relations below:

(i) The function $t_{0}(\tilde{t})$ is independent of $\eta$ and satisfies

$$
\tilde{\phi}_{J}(\tilde{t})=\phi_{\mathrm{II}}\left(t_{0}(\tilde{t})\right) \text {. }
$$

(ii) $d t_{0} / d \tilde{t}$ never vanishes on $\tilde{V}$.

(iii) The function $x_{0}(\tilde{x}, \tilde{t})$ is also independent of $\eta$ and satisfies

$$
\begin{aligned}
& x_{0}\left(\tilde{\lambda}_{0}(\tilde{t}), \tilde{t}\right)=\lambda_{0}\left(t_{0}(\tilde{t})\right), \\
& x_{0}\left(\tilde{a}_{k}(\tilde{t}), \tilde{t}\right)=a_{k}\left(t_{0}(\tilde{t})\right) \quad(k=1,2) .
\end{aligned}
$$

Here $\lambda_{0}(t)$ and $a_{k}(t)(k=1,2)$ are respectively the double and two simple turning points of $\left(S L_{\mathrm{II}}\right)$.

(iv) $\partial x_{0} / \partial \tilde{x}$ never vanishes on $\tilde{U} \times \tilde{V}$.

(v) $x_{1 / 2}$ and $t_{1 / 2}$ vanish identically.

(vi) The $\eta$-dependence of $x_{j / 2}$ and $t_{j / 2}(j \geq 2)$ is only through the instanton terms $\exp \left(\ell \tilde{\Phi}_{J}(\tilde{t}, \eta)\right)(\ell=j-2-2 m$ with $0 \leq m \leq j-2)$ that appear in the 2-parameter solution $\left(\tilde{\lambda}_{J}, \tilde{\nu}_{J}\right)$ of $\left(H_{J}\right)$.

(vii) The following relations hold:

$$
\begin{aligned}
& x\left(\tilde{\lambda}_{J}(\tilde{t}, \eta ; \tilde{\alpha}, \tilde{\beta}), \tilde{t}, \eta\right)=\lambda_{\mathrm{II}}(t(\tilde{t}, \eta), \eta ; \alpha, \beta), \\
& \tilde{Q}_{J}(\tilde{x}, \tilde{t}, \eta)=\left(\frac{\partial x(\tilde{x}, \tilde{t}, \eta)}{\partial \tilde{x}}\right)^{2} Q_{\mathrm{II}}(x(\tilde{x}, \tilde{t}, \eta), t(\tilde{t}, \eta), \eta)-\frac{1}{2} \eta^{-2}\{x(\tilde{x}, \tilde{t}, \eta) ; \tilde{x}\},
\end{aligned}
$$

where the 2-parameter solutions of $\left(H_{J}\right)$ and $\left(H_{\mathrm{II}}\right)$ are substituted into $(\lambda, \nu)$ in the coefficients of $\tilde{Q}_{J}$ and $Q_{\mathrm{II}}$, respectively, and $\{x(\tilde{x}, \tilde{t}, \eta) ; \tilde{x}\}$ denotes the Schwarzian derivative (2.41).

The rest of this section is devoted to the proof of Theorem 4.2. 


\section{§4.2. Construction of the top term of the transformation}

Here we construct the top terms $x_{0}(\tilde{x}, \tilde{t})$ and $t_{0}(\tilde{t})$ of the formal series.

First, we explain the construction of $t_{0}(\tilde{t})$. Since $\tilde{t}$ lies on a $P$-Stokes curve emanating from $\tilde{r}_{k}(k=1,2)$, it is shown in [KT1, Theorem 2.2] that there exists a function $t_{0}^{(k)}(\tilde{t})$ such that

$$
\tilde{\phi}_{J, k}(\tilde{t})=\phi_{\mathrm{II}, k}\left(t_{0}^{(k)}(\tilde{t})\right)
$$

for $k=1,2$, where

$$
\tilde{\phi}_{J, k}(\tilde{t})=\int_{\tilde{r}_{k}}^{\tilde{t}} \sqrt{\tilde{F}_{J}^{(1)}(\tilde{t})} d \tilde{t}, \quad \phi_{\mathrm{II}, k}(t)=\int_{r_{k}}^{t} \sqrt{F_{\mathrm{II}}^{(1)}(t)} d t .
$$

Here $r_{1}$ and $r_{2}$ are two simple $P$-turning points of $\left(P_{\mathrm{II}}\right)$ chosen by the following rule. We have the following two possibilities for the Stokes geometry of $\left(S L_{J}\right)$ at $\tilde{t}_{*}$ (see Figure 13):

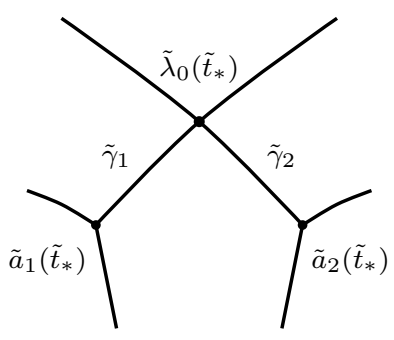

(A)

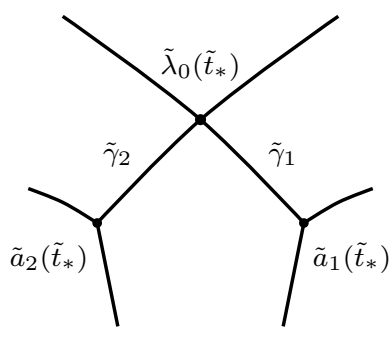

(B)

Figure 13. Two possibilities for adjacent Stokes segments of $\left(S L_{J}\right)$.

(A) The Stokes segment $\tilde{\gamma}_{2}$ follows the Stokes segment $\tilde{\gamma}_{1}$ in the counter-clockwise order near $\tilde{\lambda}_{0}\left(\tilde{t}_{*}\right)$.

(B) $\tilde{\gamma}_{2}$ follows $\tilde{\gamma}_{1}$ in the clockwise order near $\tilde{\lambda}_{0}\left(\tilde{t}_{*}\right)$.

Set

$$
\left(r_{1}, r_{2}\right)= \begin{cases}\left(r, r^{\prime}\right) & \text { when case (A) happens, } \\ \left(r^{\prime}, r\right) & \text { when case (B) happens, }\end{cases}
$$

where $r$ and $r^{\prime}$ are the $P$-turning points of $\left(P_{\mathrm{II}}\right)$ depicted in Figure $12(\mathrm{P})$. Moreover, the branch of $\sqrt{F_{\mathrm{II}}^{(1)}(t)}$ is taken so that the sign appearing on the right-hand side of (3.16) is + :

$$
\int_{r_{1}}^{r_{2}} \sqrt{F_{\mathrm{II}}^{(1)}(t)} d t=+2 \pi i c
$$

This choice (4.11) of $r_{1}$ and $r_{2}$ is essential in the construction of $x_{0}(\tilde{x}, \tilde{t})$ later. 
For each $k=1,2$, the function $t_{0}^{(k)}(\tilde{t})$ satisfying (4.9) is unique if we require that $t_{0}^{(k)}\left(\tilde{t}_{*}\right)$ lies on the $P$-Stokes segment $\Gamma$ in Figure $12(\mathrm{P})$ (cf. [KT1, Section 2, $(2.21)])$. In what follows we assume that $t_{0}^{(k)}\left(\tilde{t}_{*}\right)$ lies on $\Gamma$. Then our choice $(4.2)$ of the constant $c$ in $\left(P_{\mathrm{II}}\right)$ and (4.12) imply that

$$
\phi_{\mathrm{II}, 1}\left(t_{0}^{(k)}(\tilde{t})\right)-\phi_{\mathrm{II}, 2}\left(t_{0}^{(k)}(\tilde{t})\right)=\int_{\tilde{r}_{1}}^{\tilde{r}_{2}} \sqrt{\tilde{F}_{J}^{(1)}(\tilde{t})} d \tilde{t}=\tilde{\phi}_{J, 1}(\tilde{t})-\tilde{\phi}_{J, 2}(\tilde{t})
$$

for $k=1$, 2. In particular, we have the equality $\phi_{\mathrm{II}, 1}\left(t_{0}^{(2)}(\tilde{t})\right)=\tilde{\phi}_{J, 1}(\tilde{t})$ as the case of $k=2$ of (4.13). Since $t_{0}^{(2)}\left(\tilde{t}_{*}\right)$ lies on $\Gamma$, we have $t_{0}^{(1)}(\tilde{t})=t_{0}^{(2)}(\tilde{t})$ by the uniqueness explained above. We set $t_{0}(\tilde{t})=t_{0}^{(1)}(\tilde{t})=t_{0}^{(2)}(\tilde{t})$ and (4.4) follows from (4.9) for $k=1$. Taking a small neighborhood $\tilde{V}$ of $t_{*}$, we may assume that also the derivative $d t_{0} / d \tilde{t}$ never vanishes on $\tilde{V}$. Thus we obtain $t_{0}(\tilde{t})$ satisfying (i) and (ii) of our main claim. In particular, we have

$$
\sqrt{\tilde{F}_{J}^{(1)}(\tilde{t})}=\frac{d t_{0}(\tilde{t})}{d \tilde{t}} \sqrt{F_{\mathrm{II}}^{(1)}\left(t_{0}(\tilde{t})\right)}
$$

Next, we construct $x_{0}(\tilde{x}, \tilde{t})$. Set

$$
\left(\gamma_{1}, \gamma_{2}\right)= \begin{cases}\left(\gamma, \gamma^{\prime}\right) & \text { when case }(\mathrm{A}) \text { happens } \\ \left(\gamma^{\prime}, \gamma\right) & \text { when case }(\mathrm{B}) \text { happens }\end{cases}
$$

where $\gamma$ and $\gamma^{\prime}$ are the Stokes segments of $\left(S L_{\mathrm{II}}\right)$ (at $\left.t=t_{0}\left(\tilde{t}_{*}\right)\right)$ in Figure 12 (SL), and denote by $a_{1}(t)$ (resp., $\left.a_{2}(t)\right)$ the simple turning point of $\left(S L_{\mathrm{II}}\right)$ which is the end-point of the Stokes segment $\gamma_{1}$ (resp., $\left.\gamma_{2}\right)$ at $t=t_{0}\left(\tilde{t}_{*}\right)$. Since $\tilde{t}_{*}$ lies on a $P$-Stokes curve emanating from $\tilde{r}_{1}$, and $t_{0}(\tilde{t})$ satisfies $\phi_{J, 1}(\tilde{t})=\phi_{\mathrm{II}, 1}\left(t_{0}(\tilde{t})\right)$, the same discussion as in [KT1, Section 2] enables us to construct $x_{0}(\tilde{x}, \tilde{t})$ satisfying the following conditions:

- $x_{0}(\tilde{x}, \tilde{t})$ is holomorphic on $\tilde{U}_{1} \times \tilde{V}$, where $\tilde{U}_{1}$ is an open neighborhood of the Stokes segment $\tilde{\gamma}_{1}$ of $\left(S L_{J}\right)$, and $\partial x_{0} / \partial \tilde{x}$ never vanishes on $\tilde{U}_{1} \times \tilde{V}$.

- For any $\tilde{t} \in \tilde{V}, x_{0}(\tilde{x}, \tilde{t})$ maps $\tilde{U}_{1}$ biholomorphically to an open neighborhood $U_{1}$ of the Stokes segment $\gamma_{1}$ of $\left(S L_{\mathrm{II}}\right)$.

- Set

$$
\begin{aligned}
& \tilde{Z}_{J}(\tilde{x}, \tilde{t})=\int_{\tilde{\lambda}_{0}(\tilde{t})}^{\tilde{x}} \sqrt{\tilde{Q} \tilde{Q}_{J, 0}(\tilde{x}, \tilde{t})} d \tilde{x} \\
& Z_{\mathrm{II}}(x, \tilde{t})=\int_{\lambda_{0}\left(t_{0}(\tilde{t})\right)}^{x} \sqrt{Q_{\mathrm{II}, 0}\left(x, t_{0}(\tilde{t})\right)} d x
\end{aligned}
$$


where the branches of $\sqrt{\tilde{Q}_{J, 0}(\tilde{x}, \tilde{t})}$ and $\sqrt{Q_{\mathrm{II}, 0}(x, t)}$ are chosen so that

$$
\begin{gathered}
\int_{\tilde{\gamma}_{k}} \sqrt{\tilde{Q}_{J, 0}(\tilde{x}, \tilde{t})} d \tilde{x}=\frac{1}{2} \int_{\tilde{r}_{k}}^{\tilde{t}} \sqrt{\tilde{F}_{J}^{(1)}\left(\tilde{t}^{\prime}\right)} d \tilde{t}^{\prime}, \\
\int_{\gamma_{k}} \sqrt{Q_{\mathrm{II}, 0}(x, t)} d x=\frac{1}{2} \int_{r_{k}}^{t} \sqrt{F_{\mathrm{II}}^{(1)}\left(t^{\prime}\right)} d t^{\prime}
\end{gathered}
$$

for $k=1,2$ (cf. (3.7)). In (4.17) the Stokes segments are directed from the simple turning point to the double turning point. Then

$$
\begin{aligned}
\tilde{Z}_{J}(\tilde{x}, \tilde{t}) & =Z_{\mathrm{II}}\left(x_{0}(\tilde{x}, \tilde{t}), \tilde{t}\right), \\
x_{0}\left(\tilde{\lambda}_{0}(\tilde{t}), \tilde{t}\right) & =\lambda_{0}\left(t_{0}(\tilde{t})\right), \quad x_{0}\left(\tilde{a}_{1}(\tilde{t}), \tilde{t}\right)=a_{1}\left(t_{0}(\tilde{t})\right) .
\end{aligned}
$$

It is also shown in [KT1, Section 2] that $x_{0}(\tilde{x}, \tilde{t})$ is the unique holomorphic solution (satisfying $\left.x_{0}\left(\tilde{\lambda}_{0}(\tilde{t}), \tilde{t}\right),\left(\partial x_{0} / \partial \tilde{x}\right)\left(\tilde{\lambda}_{0}(\tilde{t}), \tilde{t}\right) \neq 0\right)$ of the implicit functional equation

$$
Z_{J}(\tilde{x}, \tilde{t})^{1 / 2}=Z_{\mathrm{II}}\left(x_{0}(\tilde{x}, \tilde{t}), \tilde{t}\right)^{1 / 2} .
$$

Here the branches of $Z_{J}(\tilde{x}, \tilde{t})^{1 / 2}$ and $Z_{\mathrm{II}}(x, \tilde{t})^{1 / 2}$ are chosen so that they are positive on $\tilde{\gamma}_{1}$ and $\gamma_{1}$, respectively, when $\tilde{t}=\tilde{t}_{*}$. Note that since we have assumed that the imaginary part of $c$ in (4.2) is positive, the real parts of $\tilde{\phi}_{J, 1}(\tilde{t})$ and $\phi_{\mathrm{II}, 1}(t)$ are decreasing along the $P$-Stokes segments $\tilde{\Gamma}$ and $\Gamma$, respectively. Then the equality (4.17) shows that the real parts of $\tilde{Z}_{J}\left(\tilde{x}, \tilde{t}_{*}\right)$ and $Z_{\mathrm{II}}\left(x, \tilde{t}_{*}\right)$ are positive along $\tilde{\gamma}_{1}$ and $\gamma_{1}$, respectively.

In view of (4.18), the four Stokes curves of $\left(S L_{J}\right)$ emanating from $\tilde{\lambda}_{0}(\tilde{t})$ are mapped by $x_{0}(\tilde{x}, \tilde{t})$ locally to those of $\left(S L_{\mathrm{II}}\right)$ emanating from $\lambda_{0}\left(t_{0}(\tilde{t})\right)$. In particular, the Stokes segment $\tilde{\gamma}_{1}$ of $\left(S L_{J}\right)$ is mapped to the Stokes segment $\gamma_{1}$ of $\left(S L_{\mathrm{II}}\right)$ when $\tilde{t}=\tilde{t}_{*}$. Furthermore, since $\partial x_{0} / \partial \tilde{x} \neq 0$ at $\tilde{x}=\tilde{\lambda}_{0}(\tilde{t})$, the other Stokes segment $\tilde{\gamma}_{2}$ is mapped to the Stokes curve emanating from $\lambda_{0}\left(t_{0}\left(\tilde{t}_{*}\right)\right)$ which follows $\gamma_{1}$ in the counter-clockwise (resp., clockwise) order in case (A) (resp., (B)) when $\tilde{t}=\tilde{t}_{*}$. Thus, our choice (4.11) of the $P$-turning points $r_{1}$ and $r_{2}$ of $\left(P_{\mathrm{II}}\right)$ entails that $x_{0}\left(\tilde{x}, \tilde{t}_{*}\right)$ maps $\tilde{\gamma}_{2}$ to the Stokes segment $\gamma_{2}$ of $\left(S L_{\mathrm{II}}\right)$ given by $(4.15)$ near $\tilde{x}=\tilde{\lambda}_{0}\left(\tilde{t}_{*}\right)$.

Since our choice (4.2) of the constant $c$ in $\left(P_{\mathrm{II}}\right)$ also ensures the equality $\phi_{J, 2}(\tilde{t})=\phi_{\mathrm{II}, 2}\left(t_{0}(\tilde{t})\right)$, the same discussion as in [KT1, Section 2] again enables us to show that $x_{0}(\tilde{x}, \tilde{t})$ is also holomorphic at the simple turning point $\tilde{a}_{2}(\tilde{t})$ and satisfies

$$
x_{0}\left(\tilde{a}_{2}(\tilde{t}), \tilde{t}\right)=a_{2}\left(t_{0}(\tilde{t})\right)
$$

Thus we have constructed $x_{0}(\tilde{x}, \tilde{t})$ satisfying the desired properties (iii) and (iv) of our main theorem. 


\section{§4.3. Transformation near the double turning point}

In this section we follow the discussion given in [KT2, Section 4]. Namely, with the aid of Theorem 2.7, we construct a pair of formal series $x^{\text {pre }}(\tilde{x}, \tilde{t}, \eta)$ and $t^{\text {pre }}(\tilde{t}, \eta)$ which transforms $\left(S L_{J}\right)$ and the deformation equation $\left(D_{J}\right)$ to $\left(S L_{\mathrm{II}}\right)$ and $\left(D_{\mathrm{II}}\right)$.

Let us first fix the correspondence of the parameters: For a given pair of parameters $(\tilde{\alpha}, \tilde{\beta})=\left(\sum_{n=0}^{\infty} \eta^{-n} \tilde{\alpha}_{n}, \sum_{n=0}^{\infty} \eta^{-n} \tilde{\beta}_{n}\right)$ of $\left(\tilde{\lambda}_{J}, \tilde{\nu}_{J}\right)$ satisfying $(2.11)$, we choose $(A(\eta), B(\eta))=\left(\sum_{n=0}^{\infty} \eta^{-n} A_{n}, \sum_{n=0}^{\infty} \eta^{-n} B_{n}\right)$ in $(2.35)$ and $(\alpha, \beta)=$ $\left(\sum_{n=0}^{\infty} \eta^{-n} \alpha_{n}, \sum_{n=0}^{\infty} \eta^{-n} \beta_{n}\right)$ in $\left(\lambda_{\mathrm{II}}, \nu_{\mathrm{II}}\right)$ so that

$$
E_{\mathrm{II}}(\alpha, \beta)=-16 A(\eta) B(\eta)=\tilde{E}_{J}(\tilde{\alpha}, \tilde{\beta}) .
$$

Here $\tilde{E}_{J}(\tilde{\alpha}, \tilde{\beta})$ and $E_{\mathrm{II}}(\alpha, \beta)$ are the formal power series defined in (2.23). Lemma 2.4 guarantees that such a choice of parameters is possible. Then the discussion in Section 2.3 enables us to construct formal series $\tilde{z}_{J}(\tilde{x}, \tilde{t}, \eta)$ and $\tilde{s}_{J}(\tilde{t}, \eta)$ (resp., $z_{\mathrm{II}}(\tilde{x}, \tilde{t}, \eta)$ and $\left.s_{\mathrm{II}}(\tilde{t}, \eta)\right)$ satisfying the properties in Theorem 2.7 for such $(A(\eta), B(\eta))$; that is,

$$
\begin{aligned}
& \sigma\left(\tilde{s}_{J}(\tilde{t}, \eta) ; A(\eta), B(\eta)\right)=\eta^{1 / 2} \tilde{z}_{J}\left(\tilde{\lambda}_{J}(\tilde{t}, \eta ; \tilde{\alpha}, \tilde{\beta}), \tilde{t}, \eta\right), \\
& \sigma\left(s_{\mathrm{II}}(t, \eta) ; A(\eta), B(\eta)\right)=\eta^{1 / 2} z_{\mathrm{II}}\left(\lambda_{\mathrm{II}}(t, \eta ; \alpha, \beta), t, \eta\right) .
\end{aligned}
$$

Similarly to [KT2, Section 4], define

$$
\begin{aligned}
x^{\mathrm{pre}}(\tilde{x}, \tilde{t}, \eta) & =z_{\mathrm{II}}^{-1}\left(\tilde{z}_{J}(\tilde{x}, \tilde{t}, \eta), s_{J}(\tilde{t}, \eta), \eta\right), \\
t^{\mathrm{pre}}(\tilde{t}, \eta) & =s_{\mathrm{II}}^{-1}\left(\tilde{s}_{J}(\tilde{t}, \eta), \eta\right) .
\end{aligned}
$$

Then each coefficient of the formal power series $x^{\text {pre }}(\tilde{x}, \tilde{t}, \eta)$ is holomorphic in $\tilde{x}$ near $\tilde{x}=\tilde{\lambda}_{0}(\tilde{t})$ and also in $\tilde{t}$ on $\tilde{V}$, and each coefficient of $t^{\text {pre }}(\tilde{t}, \eta)$ is holomorphic in $\tilde{t}$ on $\tilde{V}$. Furthermore, $x^{\text {pre }}(\tilde{x}, \tilde{t}, \eta)$ and $t^{\text {pre }}(\tilde{t}, \eta)$ have alternating parity; that is, if we denote by $\left\{x_{j / 2}^{\text {pre }}(\tilde{x}, \tilde{t}, \eta)\right\}_{j=0}^{\infty}$ (resp., $\left.\left\{t_{j / 2}^{\text {pre }}(\tilde{t}, \eta)\right\}_{j=0}^{\infty}\right)$ the coefficient of $\eta^{-j / 2}$ in the formal series (4.24) (resp., (4.25)), then:

- $x_{0}^{\text {pre }}(\tilde{x}, \tilde{t})$ and $t_{0}^{\text {pre }}(\tilde{t})$ are independent of $\eta$,

- $x_{1 / 2}^{\mathrm{pre}}$ and $t_{1 / 2}^{\mathrm{pre}}$ vanish identically,

- for $j \geq 2$, the $\eta$-dependence of $x_{j / 2}^{\mathrm{pre}}(\tilde{x}, \tilde{t}, \eta)$ and $t_{j / 2}^{\mathrm{pre}}(\tilde{t}, \eta)$ is only through the instanton terms $\exp \left(\ell \tilde{\Phi}_{J}(\tilde{t}, \eta)\right)(\ell=j-2-2 m$ with $0 \leq m \leq j-2)$.

Lemma 4.3. The top terms $x_{0}^{\mathrm{pre}}(\tilde{x}, \tilde{t})$ and $t_{0}^{\mathrm{pre}}(\tilde{t})$ coincide with $x_{0}(\tilde{x}, \tilde{t})$ and $t_{0}(\tilde{t})$ constructed in Section 4.2, respectively:

$$
x_{0}^{\mathrm{pre}}(\tilde{x}, \tilde{t})=x_{0}(\tilde{x}, \tilde{t}), \quad t_{0}^{\mathrm{pre}}(\tilde{t})=t_{0}(\tilde{t}) .
$$


Proof. It follows from (2.45) and the normalizations (4.1) and (4.3) that $t_{0}^{\text {pre }}(\tilde{t})$ satisfies

$$
\tilde{\phi}_{J, 1}(\tilde{t})=\phi_{\mathrm{II}, 1}\left(t_{0}^{\mathrm{pre}}(\tilde{t})\right) .
$$

Hence it coincides with $t_{0}(\tilde{t})$ constructed in Section 4.2. Furthermore, by choosing a branch of the square root in (2.42) appropriately, we can show that $x_{0}^{\text {pre }}(\tilde{x}, \tilde{t})$ satisfies the following conditions in a neighborhood of the Stokes segment $\tilde{\gamma}_{1}$ of $\left(S L_{J}\right)$ :

$$
\begin{gathered}
\left.x_{0}^{\mathrm{pre}}\left(\tilde{\lambda}_{0}(\tilde{t}), \tilde{t}\right)=\lambda_{0}\left(t_{0}(\tilde{t})\right), \quad\left(\partial x_{0}^{\mathrm{pre}} / \partial \tilde{x}\right)\left(\tilde{\lambda}_{0}(\tilde{t}), \tilde{t}\right)\right) \neq 0, \\
Z_{J}(\tilde{x}, \tilde{t})^{1 / 2}=Z_{\mathrm{II}}\left(x_{0}^{\mathrm{pre}}(\tilde{x}, \tilde{t}), \tilde{t}\right)^{1 / 2} .
\end{gathered}
$$

Here $Z_{J}$ and $Z_{\mathrm{II}}$ are given in (4.16), and the branches of $Z_{J}(\tilde{x}, \tilde{t})^{1 / 2}$ and $Z_{\mathrm{II}}(x, \tilde{t})^{1 / 2}$ are chosen so that they are positive on $\tilde{\gamma}_{1}$ and $\gamma_{1}$. Thus, the top term $x_{0}^{\text {pre }}(\tilde{x}, \tilde{t})$ of $x^{\text {pre }}(\tilde{x}, \tilde{t}, \eta)$ (defined by choosing an appropriate branch of $(2.42)$ ) also coincides with $x_{0}(\tilde{x}, \tilde{t})$ constructed in Section 4.2.

Therefore, the top terms of $x^{\text {pre }}(\tilde{x}, \tilde{t}, \eta)$ and $t^{\text {pre }}(\tilde{t}, \eta)$ enjoy the desired properties. Moreover, they give a local equivalence between $\left(S L_{J}\right)$ and $\left(S L_{\mathrm{II}}\right)$ together with their deformation equations $\left(D_{J}\right)$ and $\left(D_{\mathrm{II}}\right)$ near $\tilde{x}=\tilde{\lambda}_{0}(\tilde{t})$ in the following sense.

Proposition 4.4 ([KT2, Section 4]). The following equalities hold near $\tilde{x}=\tilde{\lambda}_{0}(\tilde{t})$ and $\tilde{t} \in \tilde{V}$ :

$$
\begin{aligned}
& \tilde{S}_{J, \text { odd }}(\tilde{x}, \tilde{t}, \eta)=\left(\frac{\partial x^{\text {pre }}}{\partial \tilde{x}}(\tilde{x}, \tilde{t}, \eta)\right) S_{\mathrm{II}, \text { odd }}\left(x^{\text {pre }}(\tilde{x}, \tilde{t}, \eta), t^{\text {pre }}(\tilde{t}, \eta), \eta\right) \\
& \frac{\partial x^{\text {pre }}}{\partial \tilde{t}}(\tilde{x}, \tilde{t}, \eta)=\tilde{A}_{J}(\tilde{x}, \tilde{t}, \eta) \frac{\partial x^{\text {pre }}}{\partial \tilde{x}}-A_{\mathrm{II}}\left(x^{\text {pre }}(\tilde{x}, \tilde{t}, \eta), t^{\text {pre }}(\tilde{t}, \eta), \eta\right) \frac{\partial t^{\text {pre }}}{\partial \tilde{t}}
\end{aligned}
$$

It follows from (4.27) and (4.28) that, if a WKB solution $\psi_{\mathrm{II}}(x, t, \eta)$ of $\left(S L_{\mathrm{II}}\right)$ also solves the deformation equation $\left(D_{\mathrm{II}}\right)$, then

$$
\tilde{\psi}_{J}(\tilde{x}, \tilde{t}, \eta)=\left(\frac{\partial x^{\mathrm{pre}}}{\partial \tilde{x}}(\tilde{x}, \tilde{t}, \eta)\right)^{-1 / 2} \psi_{\mathrm{II}}\left(x^{\mathrm{pre}}(\tilde{x}, \tilde{t}, \eta), t^{\mathrm{pre}}(\tilde{t}, \eta), \eta\right)
$$

is a WKB solution of $\left(S L_{J}\right)$ which also satisfies $\left(D_{J}\right)$ simultaneously near $\tilde{x}=\tilde{\lambda}_{0}(\tilde{t})$ (cf. [KT2, Proposition 3.1]).

Therefore, the formal series defined by (4.24) and (4.25) are "almost as required". However, the coefficients of $x^{\mathrm{pre}}(\tilde{x}, \tilde{t}, \eta)$ may not be holomorphic near a pair of simple turning points $\tilde{a}_{1}$ and $\tilde{a}_{2}$, for the following reason. 
The equality (4.27) tells us that the coefficient $x_{j / 2}^{\text {pre }}(\tilde{x}, \tilde{t}, \eta)(j \geq 1)$ of $x^{\text {pre }}(\tilde{x}, \tilde{t}, \eta)$ satisfies the following linear inhomogeneous differential equation:

$$
S_{-1}\left(x_{0}, t_{0}\right) \frac{\partial x_{j / 2}^{\text {pre }}}{\partial \tilde{x}}+\frac{\partial x_{0}}{\partial \tilde{x}} \frac{\partial S_{-1}}{\partial x}\left(x_{0}, t_{0}\right) x_{j / 2}^{\text {pre }}+\frac{\partial x_{0}}{\partial \tilde{x}} \frac{\partial S_{-1}}{\partial t}\left(x_{0}, t_{0}\right) t_{j / 2}^{\text {pre }}=R_{j / 2}(\tilde{x}, \tilde{t}) .
$$

Here $S_{-1}(x, t)=\sqrt{Q_{\mathrm{II}, 0}(x, t)}$ is the top term of $S_{\mathrm{II}, \text { odd }}(x, t, \eta)$ and $R_{j / 2}$ consists of the terms given by $x_{0}^{\text {pre }}, \ldots, x_{(j-1) / 2}^{\text {pre }}$. Since the coefficients of $\tilde{S}_{J \text {,odd }}(\tilde{x}, \tilde{t}, \eta)$ are singular at simple turning points, the coefficient $R_{j / 2}$ may be singular at $\tilde{x}=\tilde{a}_{1}$ and $\tilde{x}=\tilde{a}_{2}$, that is, $x_{j / 2}^{\text {pre }}$ is not holomorphic there in general.

Recall that the transformation series $\tilde{s}_{J}(\tilde{t}, \eta)$ and $s_{\mathrm{II}}(t, \eta)$ contain infinitely many free parameters as explained in Section 2.3. Thus the formal series $t^{\text {pre }}(\tilde{t}, \eta)$ also has free parameters, which will be denoted by $C_{n}$, and we write

$$
C(\eta)=\sum_{n=1}^{\infty} \eta^{-n} C_{n}
$$

Since the free parameters enter $\tilde{s}_{J}(\tilde{t}, \eta)$ and $s_{\mathrm{II}}(t, \eta)$ additively (cf. Section 2.3), the formal series $t^{\mathrm{pre}}(\tilde{t}, \eta)$ contains the free parameters in the following manner:

$$
\tilde{s}_{J}(\tilde{t}, \eta)=s_{\mathrm{II}}\left(t^{\mathrm{pre}}(\tilde{t}, \eta), \eta\right)+C(\eta) .
$$

In the subsequent subsections, we will show that, by appropriately choosing the free parameters $C_{n}$ (i.e., correcting the choices of $t_{j / 2}^{\text {pre }}$ 's in $\left.(4.29)\right), x_{j / 2}^{\text {pre }}$ 's become holomorphic in neighborhoods of both simple turning points $\tilde{x}=\tilde{a}_{1}, \tilde{a}_{2}$. The condition on $C_{n}$ 's together with the constraint (4.21) between the parameters $(\tilde{\alpha}, \tilde{\beta})$ and $(\alpha, \beta)$ gives a correspondence between 2-parameter solutions $\left(\tilde{\lambda}_{J}, \tilde{\nu}_{J}\right)$ of $\left(P_{J}\right)$ and $\left(\lambda_{\mathrm{II}}, \nu_{\mathrm{II}}\right)$ of $\left(P_{\mathrm{II}}\right)$.

\section{§4.4. Matching two transformations}

With the aid of the idea of [KT2], we show that by appropriately choosing the free parameters $C_{n}$, the coefficients $x_{j / 2}^{\text {pre }}$ of the formal series $x^{\text {pre }}(\tilde{x}, \tilde{t}, \eta)$ become holomorphic in a neighborhood of one of the two simple turning points $\tilde{x}=\tilde{a}_{1}$ and $\tilde{x}=\tilde{a}_{2}$.

The following lemma can be shown as in [AKT1] and [KT2].

Lemma 4.5 (cf. [AKT1, Lemma 2.2], [KT2, Sublemma 4.1]). For each $k=1,2$, there exist an open neighborhood $\tilde{U}_{k}^{\prime}$ of $\tilde{x}=\tilde{a}_{k}(\tilde{t})$ and a formal series

$$
y^{(k)}(\tilde{x}, \tilde{t}, \eta)=\sum_{j=0}^{\infty} \eta^{-j / 2} y_{j / 2}^{(k)}(\tilde{x}, \tilde{t}, \eta)
$$

satisfying the following conditions: 
(i) Each coefficient $y_{j / 2}^{(k)}(\tilde{x}, \tilde{t}, \eta)$ is holomorphic in $\tilde{U}_{k}^{\prime} \times \tilde{V}$.

(ii) The top term $y_{0}^{(k)}(\tilde{x}, \tilde{t})$ is independent of $\eta$, and $\partial y_{0}^{(k)} / \partial \tilde{x}$ never vanishes on $\tilde{U}_{k}^{\prime} \times \tilde{V}$.

(iii) $y_{0}^{(k)}(\tilde{x}, \tilde{t})$ satisfies $y_{0}^{(k)}\left(\tilde{a}_{k}(\tilde{t}), \tilde{t}\right)=a_{k}\left(t_{0}(\tilde{t})\right)$ and maps the Stokes segment $\tilde{\gamma}_{k}$ of $\left(S L_{J}\right)$ to the Stokes segment $\gamma_{k}$ of $\left(S L_{\mathrm{II}}\right)$ locally near $\tilde{x}=\tilde{a}_{k}(\tilde{t})$.

(iv) $y_{1 / 2}^{(k)}$ vanishes identically.

(v) For $j \geq 2$, the $\eta$-dependence of $y_{j / 2}^{(k)}(\tilde{x}, \tilde{t}, \eta)$ is only through the instanton terms $\exp \left(\ell \tilde{\Phi}_{J}(\tilde{t}, \eta)\right)(\ell=j-2-2 m$ with $0 \leq m \leq j-2)$.

(vi) The equalities

$$
\begin{aligned}
\tilde{S}_{J, \text { odd }}(\tilde{x}, \tilde{t}, \eta)= & \left(\frac{\partial y^{(k)}}{\partial \tilde{x}}(\tilde{x}, \tilde{t}, \eta)\right) S_{\mathrm{II}, \text { odd }}\left(y^{(k)}(\tilde{x}, \tilde{t}, \eta), t^{\mathrm{pre}}(\tilde{t}, \eta), \eta\right), \\
\frac{\partial y^{(k)}}{\partial \tilde{t}}(\tilde{x}, \tilde{t}, \eta)= & \tilde{A}_{J}(\tilde{x}, \tilde{t}, \eta) \frac{\partial y^{(k)}}{\partial \tilde{x}}(\tilde{x}, \tilde{t}, \eta) \\
& -A_{\mathrm{II}}\left(y^{(k)}(\tilde{x}, \tilde{t}, \eta), t^{\mathrm{pre}}(\tilde{t}, \eta), \eta\right) \frac{\partial t^{\mathrm{pre}}}{\partial \tilde{t}}
\end{aligned}
$$

hold on $\tilde{U}_{k}^{\prime} \times \tilde{V}$. Here $t^{\mathrm{pre}}(\tilde{t}, \eta)$ is given in $(4.25)$.

The top term $y_{0}^{(k)}(\tilde{x}, \tilde{t})$ is fixed as the unique holomorphic function near $\tilde{x}=$ $\tilde{a}_{k}(\tilde{t})$ satisfying

$$
\sqrt{\tilde{Q}_{J, 0}(\tilde{x}, \tilde{t})}=\left(\frac{\partial y_{0}^{(k)}}{\partial \tilde{x}}(\tilde{x}, \tilde{t})\right) \sqrt{Q_{\mathrm{II}, 0}\left(y_{0}^{(k)}(\tilde{x}, \tilde{t}), \tilde{t}\right)}
$$

at $\tilde{x}=\tilde{a}_{k}(\tilde{t})$ and condition (iii) in Lemma 4.5. Since $x_{0}(\tilde{x}, \tilde{t})$ constructed in Section 4.2 also satisfies the conditions for both $k=1,2$, we conclude that

$$
y_{0}^{(1)}(\tilde{x}, \tilde{t})=y_{0}^{(2)}(\tilde{x}, \tilde{t})=x_{0}(\tilde{x}, \tilde{t}) .
$$

Now we try to adjust the free parameters $C_{n}$ that remain in $t^{\text {pre }}(\tilde{t}, \eta)$ as described in (4.31) so that the higher order terms of the transformations $x^{\text {pre }}(\tilde{x}, \tilde{t}, \eta)$ and $y^{(1)}(\tilde{x}, \tilde{t}, \eta)$ constructed above coincide. This is a kind of "matching problem" which has been used in constructions of WKB theoretic transformations in [AKT1], [KT1], [KT2], etc.

In this subsection we denote by $y^{\text {pre }}(\tilde{x}, \tilde{t}, \eta)$ the formal series $y^{(1)}(\tilde{x}, \tilde{t}, \eta)$, and write

$$
y^{\operatorname{pre}}(\tilde{x}, \tilde{t}, \eta)=\sum_{j=0}^{\infty} \eta^{-j / 2} y_{j / 2}^{\mathrm{pre}}(\tilde{x}, \tilde{t}, \eta) \quad\left(=y^{(1)}(\tilde{x}, \tilde{t}, \eta)\right) .
$$

We note that the coefficients of the formal series $y^{(k)}(\tilde{x}, \tilde{t}, \eta)$ are holomorphic along each Stokes curve emanating from $\tilde{a}_{k}(\tilde{t})$ (cf. [AKT1, Appendix A.2]). Thus, there 
exists a domain in the $\tilde{x}$-plane on which the coefficients of both formal series $x^{\text {pre }}(\tilde{x}, \tilde{t}, \eta)$ and $y^{\text {pre }}(\tilde{x}, \tilde{t}, \eta)$ are holomorphic since the Stokes segment $\tilde{\gamma}_{1}$ connects the simple turning point $\tilde{a}_{1}(\tilde{t})$ and the double turning point $\tilde{\lambda}_{0}(\tilde{t})$ of $\left(S L_{J}\right)$ when $\tilde{t}=\tilde{t}_{*}$. In what follows we suppose that $\tilde{x}$ lies in this domain. To attain the matching, we introduce the following functions:

$$
\begin{aligned}
& \mathcal{R}(x, t, \eta)=\int_{a_{1}(t)}^{x} \eta^{-1} S_{\mathrm{II}, \text { odd }}(x, t, \eta) d x, \\
& \mathcal{F}(\tilde{x}, \tilde{t}, \eta)=\mathcal{R}\left(x^{\mathrm{pre}}(\tilde{x}, \tilde{t}, \eta), t^{\mathrm{pre}}(\tilde{t}, \eta), \eta\right), \\
& \mathcal{G}(\tilde{x}, \tilde{t}, \eta)=\mathcal{R}\left(y^{\mathrm{pre}}(\tilde{x}, \tilde{t}, \eta), t^{\mathrm{pre}}(\tilde{t}, \eta), \eta\right) .
\end{aligned}
$$

Due to the factor $\eta^{-1}$ in $(4.38), \mathcal{F}$ and $\mathcal{G}$ become formal series starting from $\eta^{0}$. It is clear from the definition (4.27) and (4.33) that

$$
\frac{\partial(\mathcal{F}-\mathcal{G})}{\partial \tilde{x}}=\eta^{-1} \tilde{S}_{J, \text { odd }}(\tilde{x}, \tilde{t}, \eta)-\eta^{-1} \tilde{S}_{J, \text { odd }}(\tilde{x}, \tilde{t}, \eta)=0 .
$$

Furthermore, using (2.22), (4.27) and (4.28), we have

$$
\begin{aligned}
\frac{\partial \mathcal{F}}{\partial \tilde{t}}= & \eta^{-1} S_{\mathrm{II}, \text { odd }}\left(x^{\mathrm{pre}}(\tilde{x}, \tilde{t}, \eta), t^{\mathrm{pre}}(\tilde{t}, \eta), \eta\right) \\
& \times\left(\frac{\partial x^{\mathrm{pre}}}{\partial \tilde{t}}(\tilde{x}, \tilde{t}, \eta)+A_{\mathrm{II}}\left(x^{\mathrm{pre}}(\tilde{x}, \tilde{t}, \eta), t^{\mathrm{pre}}(\tilde{t}, \eta), \eta\right) \frac{\partial t^{\mathrm{pre}}}{\partial \tilde{t}}(\tilde{t}, \eta)\right) \\
= & \eta^{-1} \tilde{A}_{J}(\tilde{x}, \tilde{t}, \eta) \frac{\partial x^{\mathrm{pre}}}{\partial \tilde{x}}(\tilde{x}, \tilde{t}, \eta) S_{\mathrm{II}, \text { odd }}\left(x^{\mathrm{pre}}(\tilde{x}, \tilde{t}, \eta), t^{\mathrm{pre}}(\tilde{t}, \eta), \eta\right) \\
= & \eta^{-1} \tilde{A}_{J}(\tilde{x}, \tilde{t}, \eta) \tilde{S}_{J, \text { odd }}(\tilde{x}, \tilde{t}, \eta)
\end{aligned}
$$

by a straightforward computation. In the same way we have

$$
\frac{\partial \mathcal{G}}{\partial \tilde{t}}=\eta^{-1} \tilde{A}_{J}(\tilde{x}, \tilde{t}, \eta) \tilde{S}_{J, \text { odd }}(\tilde{x}, \tilde{t}, \eta)
$$

Therefore,

$$
\frac{\partial(\mathcal{F}-\mathcal{G})}{\partial \tilde{t}}=0 .
$$

Combining (4.41) and (4.42), we conclude that

$$
\mathcal{F}-\mathcal{G}=\sum_{j=0}^{\infty} \eta^{-j / 2} \mathcal{I}_{j / 2}
$$

with genuine constants $\mathcal{I}_{j / 2}$.

Let us prove the following statement $(*)_{j}$ for any $j$ by induction on $j$ :

$(*)_{j}$ A correct choice of $t_{j / 2}^{\text {pre }}$ entails the vanishing of $\mathcal{I}_{j / 2}$ and coincidence of $x_{j / 2}^{\text {pre }}$ and $y_{j / 2}^{\text {pre }}$. 
As shown in Section 4.2 and $(4.36),(*)_{0}$ holds. Since $x_{1 / 2}^{\text {pre }}=y_{1 / 2}^{\text {pre }}=0$ and $t_{1 / 2}^{\text {pre }}=0,(*)_{1}$ is also valid. Suppose $j \geq 2$ and $(*)_{k}$ holds for all $k<j$. It follows from (4.39) and (4.40) and the induction hypothesis that

$$
\mathcal{I}_{j / 2}=S_{-1}\left(x_{0}, t_{0}\right)\left(x_{j / 2}^{\mathrm{pre}}-y_{j / 2}^{\mathrm{pre}}\right) .
$$

Here $S_{-1}(x, t)$ is the top term of $S_{\text {II,odd }}(x, t, \eta)$. On the other hand, as we have seen in (4.29), the functions $x_{j / 2}^{\text {pre }}$ and $y_{j / 2}^{\text {pre }}$ satisfy linear inhomogeneous differential equations

$$
\begin{aligned}
L x_{j / 2}^{\text {pre }} & =R\left(x_{0}^{\text {pre }}, \ldots, x_{(j-1) / 2}^{\text {pre }}, t_{0}^{\text {pre }}, \ldots, t_{(j-1) / 2}^{\text {pre }}\right), \\
L y_{j / 2}^{\text {pre }} & =R\left(y_{0}^{\text {pre }}, \ldots, y_{(j-1) / 2}^{\text {pre }}, t_{0}^{\text {pre }}, \ldots, t_{(j-1) / 2}^{\text {pre }}\right),
\end{aligned}
$$

where $L$ is a differential operator defined by

$$
\begin{aligned}
L w= & S_{-1}\left(x_{0}, t_{0}\right) \frac{\partial w}{\partial \tilde{x}}+\frac{\partial x_{0}}{\partial \tilde{x}} \frac{\partial S_{-1}}{\partial x}\left(x_{0}, t_{0}\right) w \\
& +\frac{\partial x_{0}}{\partial \tilde{x}} \frac{\partial S_{-1}}{\partial t}\left(x_{0}, t_{0}\right) t_{j / 2}^{\text {pre }},
\end{aligned}
$$

and the right-hand side of (4.45) (resp., (4.46)) is a function determined by $x_{j^{\prime} / 2}^{\text {pre }}$ (resp., $y_{j^{\prime} / 2}^{\text {pre }}$ ) and $t_{j^{\prime} / 2}^{\text {pre }}$ with $j^{\prime} \leq j-1$. The induction hypothesis implies that

$$
R\left(x_{0}^{\text {pre }}, \ldots, x_{(j-1) / 2}^{\text {pre }}, t_{0}^{\text {pre }}, \ldots, t_{(j-1) / 2}^{\text {pre }}\right)=R\left(y_{0}^{\text {pre }}, \ldots, y_{(j-1) / 2}^{\text {pre }}, t_{0}^{\text {pre }}, \ldots, t_{(j-1) / 2}^{\text {pre }}\right) .
$$

Moreover, since $x_{j / 2}^{\text {pre }}$ is non-singular near $\tilde{x}=\tilde{\lambda}_{0}(\tilde{t})$, the right-hand sides of $(4.45)$ and (4.46) must be holomorphic at $\tilde{x}=\tilde{\lambda}_{0}(\tilde{t})$. The method of variation of constants

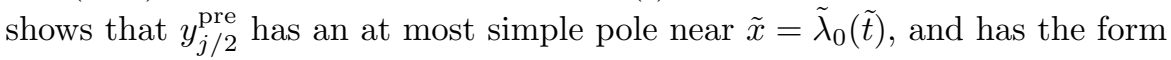

$$
y_{j / 2}^{\mathrm{pre}}(\tilde{x}, \tilde{t}, \eta)=\frac{d_{j / 2}(\tilde{t}, \eta)-t_{j / 2}^{\mathrm{pre}}(\tilde{t}, \eta)}{2\left(x_{0}(\tilde{x}, \tilde{t})-\lambda_{0}\left(t_{0}(\tilde{t})\right)\right)}+\left(\text { regular function at } \tilde{x}=\lambda_{0}(\tilde{t})\right) .
$$

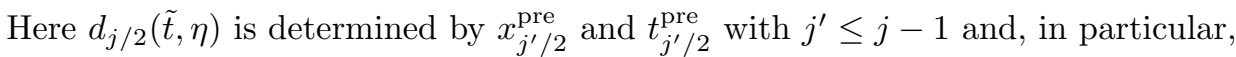
independent of $t_{j / 2}^{\text {pre }}$. Substituting (4.48) into (4.44) and taking the limit $\tilde{x} \rightarrow \tilde{\lambda}_{0}(\tilde{t})$, we obtain

$$
\frac{1}{2} \sqrt{F_{\mathrm{II}}^{(1)}\left(t_{0}(\tilde{t})\right)}\left(t_{j / 2}^{\mathrm{pre}}(\tilde{t}, \eta)-d_{j / 2}(\tilde{t}, \eta)\right)=\mathcal{I}_{j / 2} .
$$

Here we have used the equalities (2.15), (4.19),

$$
S_{-1}\left(x_{0}, t_{0}\right)=\left(x_{0}(\tilde{x}, \tilde{t})-\lambda_{0}\left(t_{0}(\tilde{t})\right)\right) \sqrt{R_{\mathrm{II}}\left(x_{0}(\tilde{x}, \tilde{t}), t_{0}(\tilde{t})\right)},
$$

and the fact that $x_{j / 2}^{\text {pre }}(\tilde{x}, \tilde{t}, \eta)$ is holomorphic at $\tilde{x}=\tilde{\lambda}_{0}(\tilde{t})$. Again we emphasize that $\mathcal{I}_{j / 2}$ is independent of $\tilde{t}$. 
Suppose that $j$ is even, $j=2 n(n \geq 1)$. Then, in view of $(4.31)$, the free parameter $C_{n}$ remains in $t_{j / 2}^{\text {pre }}(\tilde{t}, \eta)=t_{n}^{\text {pre }}(\tilde{t}, \eta)$ in the form

$$
t_{n}^{\text {pre }}(\tilde{t}, \eta)=\left(\frac{d s_{0}}{d t}\left(t_{0}(\tilde{t})\right)\right)^{-1} C_{n}+N(\tilde{t}, \eta) .
$$

Here $s_{0}(t)$ is the top term (2.45) of the formal series $s_{\mathrm{II}}(t, \eta)$ and hence

$$
\frac{d s_{0}}{d t}\left(t_{0}(\tilde{t})\right)=\frac{1}{2} \sqrt{F_{\mathrm{II}}^{(1)}\left(t_{0}(\tilde{t})\right)}
$$

is non-zero, at least when $\tilde{t}=\tilde{t}_{*}$. The term $N(\tilde{t}, \eta)$ in (4.50) consists of terms which are independent of $C_{n}$. Thus, (4.49) and (4.50) show that a suitable choice of the free parameter $C_{n}$ makes $\mathcal{I}_{j / 2}=\mathcal{I}_{n}$ vanish. Hence (4.44) implies

$$
x_{j / 2}^{\text {pre }}(\tilde{x}, \tilde{t}, \eta)=y_{j / 2}^{\text {pre }}(\tilde{x}, \tilde{t}, \eta),
$$

that is, the claim $(*)_{j}$.

Next we consider the case where $j$ is odd. In this case, by alternating parity, $\mathcal{I}_{j / 2}$ must contain only odd instanton terms, and hence it never contains constant terms. Thus $\mathcal{I}_{j / 2}$ must vanish, and (4.44) implies (4.51).

Thus $(*)_{j}$ is valid for every $j$. In other words, the formal series $x^{\text {pre }}(\tilde{x}, \tilde{t}, \eta)$ and $y^{\operatorname{pre}}(\tilde{x}, \tilde{t}, \eta)$ coincide after the correct choice of free parameters:

$$
x^{\text {pre }}(\tilde{x}, \tilde{t}, \eta)=y^{\operatorname{pre}}(\tilde{x}, \tilde{t}, \eta) \quad\left(=y^{(1)}(\tilde{x}, \tilde{t}, \eta)\right) .
$$

Since all the free parameters in $t^{\text {pre }}(\tilde{t}, \eta)$ have been fixed, the correspondence of parameters between $(\tilde{\alpha}(\eta), \tilde{\beta}(\eta))$ and $(\alpha(\eta), \beta(\eta))$ is also fixed. In what follows we always assume that the parameters are chosen so that (4.52) holds, and denote by

$$
t(\tilde{t}, \eta)=\sum_{j=0}^{\infty} \eta^{-j / 2} t_{j / 2}(\tilde{t}, \eta)
$$

the formal series $t^{\text {pre }}$ after the correct choice of the free parameters. In the next subsection, we will show that the formal series (4.52) also coincides with $y^{(2)}(\tilde{x}, \tilde{t}, \eta)$ and consequently the coefficients of (4.52) are also holomorphic near the simple turning point $\tilde{x}=\tilde{a}_{2}(\tilde{t})$.

\section{§4.5. Transformation near the pair of two simple turning points and transformation of 2-parameter solutions}

Finally, in this subsection we show that the formal series $y^{(1)}(\tilde{x}, \tilde{t}, \eta)$ and $y^{(2)}(\tilde{x}, \tilde{t}, \eta)$ constructed in Lemma 4.5 coincide. Our choice (4.2) of the constant $c$ in $\left(P_{\mathrm{II}}\right)$ and $\left(S L_{\mathrm{II}}\right)$ enables us to show the following claim. 
Proposition 4.6. The formal series $y^{(1)}(\tilde{x}, \tilde{t}, \eta)$ and $y^{(2)}(\tilde{x}, \tilde{t}, \eta)$ constructed in Lemma 4.5 coincide:

$$
y^{(1)}(\tilde{x}, \tilde{t}, \eta)=y^{(2)}(\tilde{x}, \tilde{t}, \eta) .
$$

Consequently, the coefficients of $y^{(1)}(\tilde{x}, \tilde{t}, \eta)$ and $y^{(2)}(\tilde{x}, \tilde{t}, \eta)$ are holomorphic in $\tilde{x}$ on a domain containing the pair of simple turning points $\tilde{x}=\tilde{a}_{1}(\tilde{t}), \tilde{a}_{2}(\tilde{t})$ of $\left(S L_{J}\right)$.

Proof. We assume that case (A) in Figure 13 happens. The discussion below is also applicable to case (B). Moreover, we will show (4.54) for $\tilde{t}=\tilde{t}_{*}$. This is just for the sake of clarity, and our proof is also valid for any $\tilde{t}$ in a neighborhood $\tilde{V}$ of $\tilde{t}_{*}$. (We may take a smaller neighborhood $\tilde{V}$ if necessary.)

By $(4.52)$ the coefficients of $y^{(1)}(\tilde{x}, \tilde{t}, \eta)$ are holomorphic in $\tilde{x}$ near $\tilde{\lambda}_{0}(\tilde{t})$. Therefore, there exists a domain $\tilde{U}^{\prime}$ containing a part of the Stokes segment $\tilde{\gamma}_{2}$ on which the coefficients of both $y^{(1)}(\tilde{x}, \tilde{t}, \eta)$ and $y^{(2)}(\tilde{x}, \tilde{t}, \eta)$ are holomorphic because $\tilde{\gamma}_{2}$ connects $\tilde{a}_{2}\left(\tilde{t}_{*}\right)$ and $\tilde{\lambda}_{0}\left(\tilde{t}_{*}\right)$. In the proof of Proposition 4.6 we assume that $\tilde{x}$ lies in the domain $\tilde{U}^{\prime}$. Note that the top terms $y_{0}^{(1)}(\tilde{x}, \tilde{t})$ and $y_{0}^{(2)}(\tilde{x}, \tilde{t})$ coincide and are holomorphic in $\tilde{U}^{\prime}$ as we have seen in (4.36).

Using (4.33), we have

$$
\int_{\tilde{\delta}_{\tilde{x}}^{(k)}} \tilde{S}_{J, \text { odd }}(\tilde{x}, \tilde{t}, \eta) d \tilde{x}=\left.\int_{\delta_{x}^{(k)}} S_{\text {II,odd }}(x, t(\tilde{t}, \eta), \eta) d x\right|_{x=y^{(k)}(\tilde{x}, \tilde{t}, \eta)}
$$

for $k=1,2$ (cf. [KT3, Section 2]). Here the integration path $\tilde{\delta}_{\tilde{x}}^{(k)}$ is a contour in the domain $\tilde{U}_{k}^{\prime}$ depicted in Figure 14. That is, $\tilde{\delta}_{\tilde{x}}^{(k)}$ starts from the point on the second sheet of the Riemann surface of $\sqrt{Q_{J, 0}(\tilde{x}, \tilde{t})}$ corresponding to $\tilde{x}$, encircles the simple turning point $\tilde{a}_{k}\left(\tilde{t}_{*}\right)$ and ends at the point corresponding to $\tilde{x}$ on the first sheet. (The wiggly line designates the branch cut for $\sqrt{Q_{J, 0}(\tilde{x}, \tilde{t})}$.) The path

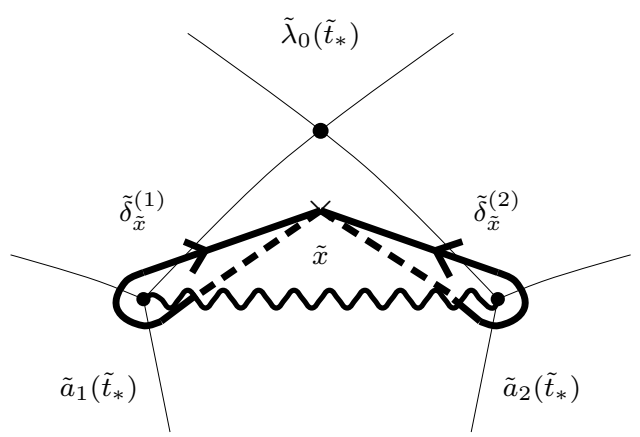

Figure 14. The paths $\tilde{\delta}_{\tilde{x}}^{(k)}$. 
$\delta_{x}^{(k)}$ is defined in the same manner for $J=$ II. The right-hand side of $(4.55)$ is written as

$$
\begin{aligned}
\int_{\delta_{x}^{(k)}} S_{\mathrm{II}, \text { odd }}(x, t(\tilde{t}, \eta), \eta) & \left.d x\right|_{x=y^{(k)}}=\left.\int_{\delta_{x}^{(k)}} S_{\mathrm{II}, \text { odd }}(x, t(\tilde{t}, \eta), \eta) d x\right|_{x=y_{0}^{(k)}} \\
& +\sum_{n=0}^{\infty} \frac{\partial^{n} S_{\mathrm{II}, \text { odd }}}{\partial x^{n}}\left(y_{0}^{(k)}, t(\tilde{t}, \eta), \eta\right) \frac{\left(y^{(k)}-y_{0}^{(k)}\right)^{n+1}}{(n+1) !}
\end{aligned}
$$

by (formal) Taylor expansion. Taking the difference of both sides of (4.55) for $k=1$ and $k=2$, we have

$$
\begin{aligned}
\int_{\tilde{\delta}^{\prime}} \tilde{S}_{J, \text { odd }}(\tilde{x}, \tilde{t}, \eta) d \tilde{x}=\int_{\delta^{\prime}} S_{\text {II }, \text { odd }}(x, t(\tilde{t}, \eta), \eta) d x \\
+\sum_{n=0}^{\infty} \frac{\partial^{n} S_{\text {II,odd }}}{\partial x^{n}}\left(x_{0}, t(\tilde{t}, \eta), \eta\right) \frac{\left(y^{(2)}-x_{0}\right)^{n+1}-\left(y^{(1)}-x_{0}\right)^{n+1}}{(n+1) !}
\end{aligned}
$$

where $\tilde{\delta}^{\prime}$ is a closed path in $\tilde{U}_{1}^{\prime} \cup \tilde{U}_{2}^{\prime}$ which encircles the pair of simple turning points $\tilde{a}_{1}\left(\tilde{t}_{*}\right)$ and $\tilde{a}_{2}\left(\tilde{t}_{*}\right)$ as indicated in Figure $15\left(\delta^{\prime}\right.$ is defined in the same manner for $J=\mathrm{II})$. Here we have used the equality (4.36).
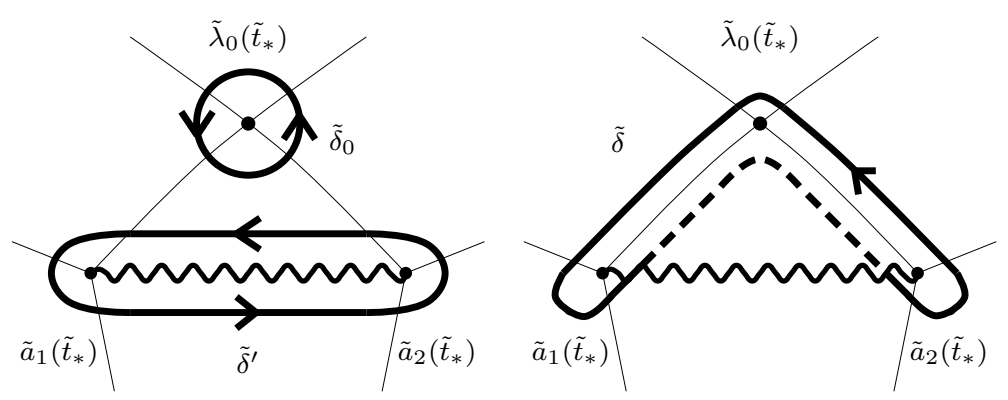

Figure 15 . The cycles $\tilde{\delta}^{\prime}, \tilde{\delta}_{0}$ and $\tilde{\delta}$.

Now we prove the following key lemma.

Lemma 4.7. If the constant $c$ in $\left(P_{\mathrm{II}}\right)$ and $\left(S L_{\mathrm{II}}\right)$ is chosen by $(4.2)$ and the free parameters satisfy (4.21), then

$$
\int_{\tilde{\delta}^{\prime}} \tilde{S}_{J, \text { odd }}(\tilde{x}, \tilde{t}, \eta) d \tilde{x}=\int_{\delta^{\prime}} S_{\mathrm{II}, \text { odd }}(x, t, \eta) d x .
$$

The left-hand (resp., right-hand) side of (4.58) does not depend on $\tilde{t}$ (resp., $t$ ), and hence (4.58) is an equality for constants. 
Proof. Let $\tilde{\delta}$ be a closed cycle encircling two Stokes segments $\tilde{\gamma}_{1}$ and $\tilde{\gamma}_{2}$ as indicated in Figure 15, and $\delta$ be a similar cycle for $J=$ II. The cycles can be decomposed as $\tilde{\delta}^{\prime}=\tilde{\delta}-\tilde{\delta}_{0}$ and $\delta^{\prime}=\delta-\delta_{0}$, where $\tilde{\delta}_{0}$ is a closed cycle encircling the double turning point $\tilde{\lambda}_{0}\left(\tilde{t}_{*}\right)$ as in Figure 15 , and $\delta_{0}$ is defined in the same manner for $J=$ II. Then (4.21) implies that

$$
\int_{\tilde{\delta}_{0}} \tilde{S}_{J, \text { odd }}(\tilde{x}, \tilde{t}, \eta) d \tilde{x}=\int_{\delta_{0}} S_{\text {II }, \text { odd }}(x, t, \eta) d x
$$

since $\tilde{E}_{J} / 4$ and $E_{\mathrm{II}} / 4$ are the residues of $\tilde{S}_{J, \text { odd }}(\tilde{x}, \tilde{t}, \eta) d \tilde{x}$ and $S_{\mathrm{II}, \text { odd }}(x, t, \eta) d x$ at the double turning points $\tilde{\lambda}_{0}(\tilde{t})$ and $\lambda_{0}\left(t_{0}(\tilde{t})\right)$, respectively. In particular, both sides of (4.59) are independent of $\tilde{t}$ and $t$.

Furthermore, our choice (4.2) of the constant $c$ in $\left(P_{\mathrm{II}}\right)$ and $\left(S L_{\mathrm{II}}\right)$ entails that

$$
\int_{\tilde{\delta}} \tilde{S}_{J, \text { odd }}(\tilde{x}, \tilde{t}, \eta) d \tilde{x}=\int_{\delta} S_{\text {II,odd }}(x, t, \eta) d x
$$

for the following reason.

First, since we assume that all singular points of $\tilde{Q}_{J, 0}(\tilde{x}, \tilde{t})$ are poles of even order in Assumption 4.1(5), $\sqrt{\tilde{Q}_{J, 0}(\tilde{x}, \tilde{t})}$ (and hence $\tilde{S}_{J, \text { odd }}(\tilde{x}, \tilde{t}, \eta)$ ) does not have branch points except for $\tilde{a}_{1}(\tilde{t})$ and $\tilde{a}_{2}(\tilde{t})$. Therefore, the left-hand side of (4.60) reduces to the sum of the residues of $\tilde{S}_{J, \text { odd }}(\tilde{x}, \tilde{t}, \eta) d \tilde{x}$ at singular points of $\left(S L_{J}\right)$. As is noted in (2.31), these residues coincide with those of $\eta \sqrt{\tilde{Q}_{J, 0}(\tilde{x}, \tilde{t})} d \tilde{x}$. Thus we have

$$
\int_{\tilde{\delta}} \tilde{S}_{J, \text { odd }}(\tilde{x}, \tilde{t}, \eta) d \tilde{x}=\eta \int_{\tilde{\delta}} \sqrt{\tilde{Q}_{J, 0}(\tilde{x}, \tilde{t})} d \tilde{x} .
$$

On the other hand, the equality (4.17) shows that

$$
\begin{aligned}
\oint_{\tilde{\delta}} \sqrt{\tilde{Q}_{J, 0}(\tilde{x}, \tilde{t})} d \tilde{x} & =2\left(\int_{\tilde{\gamma}_{1}} \sqrt{\tilde{Q}_{J, 0}(\tilde{x}, \tilde{t})} d \tilde{x}-\int_{\tilde{\gamma}_{2}} \sqrt{\tilde{Q}_{J, 0}(\tilde{x}, \tilde{t})} d \tilde{x}\right) \\
& =\int_{\tilde{r}_{1}}^{\tilde{r}_{2}} \sqrt{\tilde{F}_{J}^{(1)}(\tilde{t})} d \tilde{t}=2 \pi i c .
\end{aligned}
$$

Here we have used (4.2). Since (4.61) and (4.62) also hold for $J=$ II, we have

$$
\int_{\delta} S_{\mathrm{II}, \mathrm{odd}}(x, t, \eta) d x=2 \pi i c \eta .
$$

Combining (4.61)-(4.63), we obtain

$$
\int_{\tilde{\delta}} \tilde{S}_{J, \text { odd }}(\tilde{x}, \tilde{t}, \eta) d \tilde{x}=2 \pi i c \eta=\int_{\delta} S_{\mathrm{II}, \text { odd }}(x, t, \eta) d x,
$$

which proves (4.60). 
As is explained above, we have

$$
\begin{aligned}
\int_{\tilde{\delta}^{\prime}} \tilde{S}_{J, \text { odd }}(\tilde{x}, \tilde{t}, \eta) d \tilde{x} & =\int_{\tilde{\delta}} \tilde{S}_{J, \text { odd }}(\tilde{x}, \tilde{t}, \eta) d \tilde{x}-\int_{\tilde{\delta}_{0}} \tilde{S}_{J, \text { odd }}(\tilde{x}, \tilde{t}, \eta) d \tilde{x}, \\
\int_{\delta^{\prime}} S_{\text {II }, \text { odd }}(x, t, \eta) d x & =\int_{\delta} S_{\text {II,odd }}(x, t, \eta) d x-\int_{\delta_{0}} S_{\text {II,odd }}(x, t, \eta) d x .
\end{aligned}
$$

Therefore, the desired equality (4.58) follows from (4.59) and (4.60).

By Lemma 4.7, the equality (4.57) implies that

$$
\sum_{n=0}^{\infty} \frac{\partial^{n} S_{\mathrm{II}, \text { odd }}}{\partial x^{n}}\left(x_{0}, t(\tilde{t}, \eta), \eta\right) \frac{\left(y^{(2)}-x_{0}\right)^{n+1}-\left(y^{(1)}-x_{0}\right)^{n+1}}{(n+1) !}=0 .
$$

The coefficient of $\eta^{-(j-2) / 2}$ on the left-hand side of (4.65) can be written as

$$
S_{-1}\left(x_{0}, t_{0}\right)\left(y_{j / 2}^{(2)}-y_{j / 2}^{(1)}\right)+\left(T_{j / 2}\left(y_{0}^{(2)}, \ldots, y_{(j-1) / 2}^{(2)}\right)-T_{j / 2}\left(y_{0}^{(1)}, \ldots, y_{(j-1) / 2}^{(1)}\right)\right),
$$

where $S_{-1}(x, t)$ is the top term of $S_{\text {II,odd }}(x, t, \eta)$, and $T_{j / 2}\left(y_{0}^{(2)}, \ldots, y_{(j-1) / 2}^{(2)}\right)$ (resp., $\left.T_{j / 2}\left(y_{0}^{(1)}, \ldots, y_{(j-1) / 2}^{(1)}\right)\right)$ consists of the terms given by $y_{0}^{(2)}, \ldots, y_{(j-1) / 2}^{(2)}$ (resp., $\left.y_{0}^{(1)}, \ldots, y_{(j-1) / 2}^{(1)}\right)$. Hence we can prove $y_{j / 2}^{(1)}(\tilde{x}, \tilde{t}, \eta)=y_{j / 2}^{(2)}(\tilde{x}, \tilde{t}, \eta)$ for all $j \geq 0$ by induction.

Set

$$
x(\tilde{x}, \tilde{t}, \eta)=x^{\operatorname{pre}}(\tilde{x}, \tilde{t}, \eta) \quad\left(=y^{(1)}(\tilde{x}, \tilde{t}, \eta)=y^{(2)}(\tilde{x}, \tilde{t}, \eta)\right) .
$$

We have proved that the coefficients of the formal series $x(\tilde{x}, \tilde{t}, \eta)$ are holomorphic in a domain $\tilde{U}$ containing the double turning point $\tilde{\lambda}_{0}(\tilde{t})$ and the pair of simple turning points $\tilde{a}_{1}(\tilde{t})$ and $\tilde{a}_{2}(\tilde{t})$. The formal series $x(\tilde{x}, \tilde{t}, \eta)$ and $t(\tilde{t}, \eta)$ have almost all the properties desired in Theorem 4.2.

It remains to prove (4.7). This is a consequence of Proposition 4.4; in fact, (4.28) reads

$$
\begin{aligned}
& 2 \tilde{B}_{J}(\tilde{x}, \tilde{t}, \eta) \frac{x(\tilde{x}, \tilde{t}, \eta)-\lambda_{\mathrm{II}}(t(\tilde{t}, \eta), \eta)}{\tilde{x}-\tilde{\lambda}_{J}(\tilde{t}, \eta)} \frac{\partial x}{\partial \tilde{x}} \\
&=\frac{\partial t}{\partial \tilde{t}}+2\left(x(\tilde{x}, \tilde{t}, \eta)-\lambda_{\mathrm{II}}(t(\tilde{t}, \eta), \eta) \frac{\partial x}{\partial \tilde{t}} .\right.
\end{aligned}
$$

Here $\tilde{B}_{J}(\tilde{x}, \tilde{t}, \eta)$ is defined to be $\left(\tilde{x}-\tilde{\lambda}_{J}(\tilde{t}, \eta)\right) \tilde{A}_{J}(\tilde{x}, \tilde{t}, \eta)$, which is holomorphic at $\tilde{x}=\tilde{\lambda}_{J}(\tilde{t}, \eta)$ in view of Table 5 . Since the right-hand side of (4.67) is non-singular at $\tilde{x}=\tilde{\lambda}_{J}(\tilde{t}, \eta)$, we find

$$
x\left(\tilde{\lambda}_{J}(\tilde{t}, \eta), \tilde{t}, \eta\right)=\lambda_{\mathrm{II}}(t(\tilde{t}, \eta), \eta) .
$$

Thus we have proved all claims in Theorem 4.2. 


\section{$\S 5$. Transformation to $\left(P_{\mathrm{III}^{\prime}\left(D_{7}\right)}\right)$ on loop-type $P$-Stokes segments}

In this section we show our second main claim concerning WKB theoretic transformation of Painlevé transcendents on a loop-type $P$-Stokes segment. We put over variables or functions relevant to $\left(P_{J}\right)$ and $\left(S L_{J}\right)$ as in the previous section.

\section{§5.1. Assumptions and statements}

Let $\left(\tilde{\lambda}_{J}, \tilde{\nu}_{J}\right)=\left(\tilde{\lambda}_{J}(\tilde{t}, \eta ; \tilde{\alpha}, \tilde{\beta}), \tilde{\nu}_{J}(\tilde{t}, \eta ; \tilde{\alpha}, \tilde{\beta})\right)$ be a 2-parameter solution of $\left(H_{J}\right)$ defined in a neighborhood of a point $\tilde{t}_{*} \in \Omega_{J}$, and consider $\left(S L_{J}\right)$ and $\left(D_{J}\right)$ with $\left(\tilde{\lambda}_{J}, \tilde{\nu}_{J}\right)$ substituted into their coefficients. In this section we impose the following conditions.

Assumption 5.1. (1) $J \in\left\{\mathrm{III}^{\prime}\left(D_{7}\right), \mathrm{III}^{\prime}\left(D_{6}\right), \mathrm{IV}, \mathrm{V}, \mathrm{VI}\right\}$.

(2) There is a $P$-Stokes segment $\tilde{\Gamma}$ of loop type in the $P$-Stokes geometry of $\left(P_{J}\right)$ which emanates from and returns to a simple $P$-turning point $\tilde{r}$ of $\tilde{\lambda}_{J}$ (which is not of simple-pole type), and the point $\tilde{t}_{*}$ in question lies on $\tilde{\Gamma}$ as indicated in Figure 16(a).

(3) The function (2.9) appearing in the instanton $\tilde{\Phi}_{J}(\tilde{t}, \eta)$ of the 2-parameter solution $\left(\tilde{\lambda}_{J}, \tilde{\nu}_{J}\right)$ is normalized at the simple $P$-turning point $\tilde{r}$ :

$$
\tilde{\phi}_{J}(\tilde{t})=\int_{\tilde{r}}^{\tilde{t}} \sqrt{\tilde{F}_{J}^{(1)}(\tilde{t})} d \tilde{t} .
$$

Here the integration path is taken along one of the paths $\tilde{\Gamma}_{\tilde{t}, 1}$ or $\tilde{\Gamma}_{\tilde{t}, 2}$ shown in Figure 16(a). (Since there are singular points inside a loop-type $P$-Stokes segment in general, the two paths $\tilde{\Gamma}_{\tilde{t}, 1}$ and $\tilde{\Gamma}_{\tilde{t}, 2}$ are not homotopic in general.)

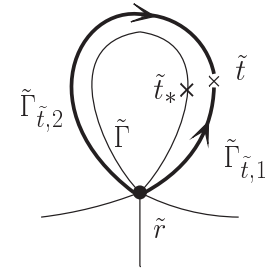

(a): The loop-type $P$-Stokes segment $\tilde{\Gamma}$

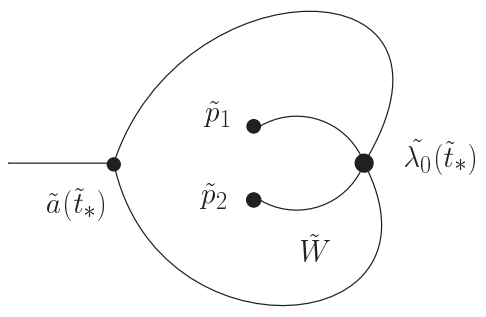

(b): The Stokes geometry of $\left(S L_{J}\right)$ at

Figure 16. The $P$-Stokes geometry of $\left(P_{J}\right)$ and an example of the Stokes geometry of $\left(S L_{J}\right)$ satisfying Assumption 5.1.

(4) The Stokes geometry of $\left(S L_{J}\right)$ at $\tilde{t}=\tilde{t}_{*}$ contains the same configuration as in Figure 16(b). That is, the following conditions hold: 
- The double turning point $\tilde{\lambda}_{0}\left(\tilde{t}_{*}\right)$ is connected to the same simple turning point $\tilde{a}\left(\tilde{t}_{*}\right)$ by two Stokes segments $\tilde{\gamma}_{1}$ and $\tilde{\gamma}_{2}$. Here the labels of the Stokes segments are given as follows: When $\tilde{t}$ tends to $\tilde{r}$ along the path $\tilde{\Gamma}_{\tilde{t}, 1}$ (resp., $\tilde{\Gamma}_{\tilde{t}, 2}$ ) depicted in Figure 16(a), the Stokes segment $\tilde{\gamma}_{1}$ (resp., $\tilde{\gamma}_{2}$ ) shrinks to a point (cf. Proposition 3.5).

- The union of the Stokes segments $\tilde{\gamma}_{1}$ and $\tilde{\gamma}_{2}$ divides the $\tilde{x}$-plane into two domains. Let $\tilde{W}$ be the one which contains the end-points $\tilde{p}_{1}$ and $\tilde{p}_{2}$ of two Stokes curves of $\left(S L_{J}\right)$ emanating from $\tilde{\lambda}_{0}(\tilde{t})$ other than $\tilde{\gamma}_{1}$ or $\tilde{\gamma}_{2}$. Then the end-point of the Stokes curve emanating from $\tilde{a}\left(\tilde{t}_{*}\right)$ is not contained in the domain $\tilde{W}$. (Unlike in Figure $16(\mathrm{~b})$, the domain $\tilde{W}$ may contain $\tilde{x}=\infty$. Also, the points $\tilde{p}_{1}$ and $\tilde{p}_{2}$ may coincide.)

(5) The domain $\tilde{W}$ defined above contains no turning point of $\left(S L_{J}\right)$ other than $\tilde{a}(\tilde{t})$ and $\tilde{\lambda}_{0}(\tilde{t})$. All singular points of $\tilde{Q}_{J, 0}(\tilde{x}, \tilde{t})$ (as a function of $\tilde{x}$ ) contained in $\tilde{W}$ are poles of even order.

Since the $P$-Stokes geometry for $J=\mathrm{I}$, II, and $\operatorname{III}^{\prime}\left(D_{8}\right)$ never contains a $P$-Stokes segment of loop type, we have excluded these cases. Similarly to Theorem 4.2, under Assumption 5.1 we will construct a formal transformation series to the third Painlevé equation $\left(P_{\mathrm{III}^{\prime}\left(D_{7}\right)}\right)$ of type $D_{7}$.

We fix the constant $c$ contained in $\left(P_{\mathrm{III}^{\prime}\left(D_{7}\right)}\right)$ and $\left(S L_{\mathrm{III}^{\prime}\left(D_{7}\right)}\right)$ by

$$
c=\frac{1}{2 \pi i} \int_{\tilde{\Gamma}} \sqrt{\tilde{F}_{J}^{(1)}(\tilde{t})} d \tilde{t},
$$

where the integration path is taken along the loop-type $P$-Stokes segment $\tilde{\Gamma}$ in the same direction as the integral (5.1); that is, when (5.1) is defined along the path $\tilde{\Gamma}_{\tilde{t}, 1}$ (resp., $\tilde{\Gamma}_{\tilde{t}, 2}$ ) in Figure $16(\mathrm{a})$, then the integration path in $(5.2)$ is taken in the counter-clockwise (resp., clockwise) direction along $\tilde{\Gamma}$. Here we assume that $c \in i \mathbb{R}_{>0}$. The $P$-Stokes geometry of $\left(P_{\mathrm{III}^{\prime}\left(D_{7}\right)}\right)$ (described in the variable $u$ given by (3.11)) is the same as in Figure $17(\mathrm{P})$ when $c$ is given by (5.2). Thus, the $P$-Stokes geometry of $\left(P_{\mathrm{III}^{\prime}\left(D_{7}\right)}\right)$ has a loop-type $P$-Stokes segment $\Gamma$ starting from and returning to the same $P$-simple turning point $r$. (As is remarked in Section 3.3, when $c \in i \mathbb{R}_{<0}$, the $P$-Stokes geometry of $\left(P_{\mathrm{III}^{\prime}\left(D_{7}\right)}\right)$ is the reflection $u \mapsto-u$ of Figure 17 $(\mathrm{P})$, and our discussion below also applies to the case $c \in i \mathbb{R}_{<0}$.) Furthermore, we can verify that the corresponding Stokes geometry of $\left(S L_{\mathrm{III}^{\prime}\left(D_{7}\right)}\right)$ on the loop-type $P$-Stokes segment $\Gamma$ is the same as the Stokes geometry in Figure 17 (SL). That is, when a point $t$ lies on $\Gamma$, the corresponding Stokes geometry of $\left(S L_{\mathrm{III}^{\prime}\left(D_{7}\right)}\right)$ has a double turning point $x=\lambda_{0}(t)$ and a simple turning point $x=a(t)$, and two Stokes segments $\gamma$ and $\gamma^{\prime}$, both connecting $\lambda_{0}(t)$ and $a(t)$. These Stokes segments are labeled as follows: When $t$ tends to $r$ along the path $\Gamma_{t}\left(\right.$ resp., $\left.\Gamma_{t}^{\prime}\right)$ depicted in 


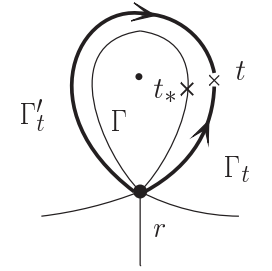

(P): The P-Stokes geometry of $\left(P_{\mathrm{III}^{\prime}\left(D_{7}\right)}\right)$ (described on the $u$-plane)

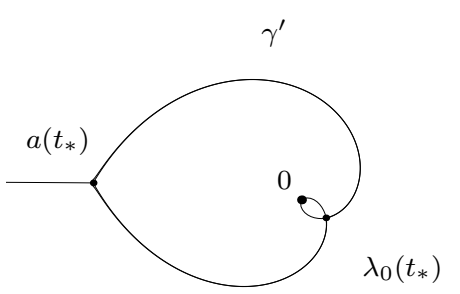

$\gamma$

(SL): The Stokes geomtry of $\left(S L_{\mathrm{III}\left(D_{7}\right)}\right)$ at $t=t_{*}$

Figure 17. The $P$-Stokes geometry of $\left(P_{\mathrm{III}^{\prime}\left(D_{7}\right)}\right)$ and the Stokes geometry of $\left(S L_{\mathrm{III}\left(D_{7}\right)}\right)$.

Figure $17(\mathrm{P})$, the Stokes segment $\gamma$ (resp., $\gamma^{\prime}$ ) shrinks to a point (cf. Proposition $3.5)$.

Having the above geometric properties in mind, we formulate our second main result.

Theorem 5.2. Under Assumption 5.1, for any 2-parameter solution $\left(\tilde{\lambda}_{J}, \tilde{\nu}_{J}\right)=$ $\left(\tilde{\lambda}_{J}(\tilde{t}, \eta ; \tilde{\alpha}, \tilde{\beta}), \tilde{\nu}_{J}(\tilde{t}, \eta ; \tilde{\alpha}, \tilde{\beta})\right)$ of $\left(H_{J}\right)$, there exist

- an annular domain $\tilde{U}$ which contains the union $\tilde{\gamma}_{1} \cup \tilde{\gamma}_{2}$ of two Stokes segments,

- a neighborhood $\tilde{V}$ of $\tilde{t}_{*}$,

- formal series

$$
x(\tilde{x}, \tilde{t}, \eta)=\sum_{j \geq 0} \eta^{-j / 2} x_{j / 2}(\tilde{x}, \tilde{t}, \eta), \quad t(\tilde{t}, \eta)=\sum_{j \geq 0} \eta^{-j / 2} t_{j / 2}(\tilde{t}, \eta)
$$

whose coefficients $\left\{x_{j / 2}(\tilde{x}, \tilde{t}, \eta)\right\}_{j=0}^{\infty}\left\{t_{j / 2}(\tilde{t}, \eta)\right\}_{j=0}^{\infty}$ are functions defined on $\tilde{U} \times \tilde{V}$ and $\tilde{V}$, respectively, and may depend on $\eta$,

- a 2-parameter solution

$$
\begin{aligned}
\left(\lambda_{\mathrm{III}\left(D_{7}\right)}, \nu_{\mathrm{III}^{\prime}\left(D_{7}\right)}\right) & =\left(\lambda_{\mathrm{III}^{\prime}\left(D_{7}\right)}(t, \eta ; \alpha, \beta), \nu_{\mathrm{III}^{\prime}\left(D_{7}\right)}(t, \eta ; \alpha, \beta)\right), \\
(\alpha, \beta) & =\left(\sum_{n=0}^{\infty} \eta^{-n} \alpha_{n}, \sum_{n=0}^{\infty} \eta^{-n} \beta_{n}\right)
\end{aligned}
$$

of $\left(H_{\mathrm{III}^{\prime}\left(D_{7}\right)}\right)$ with the constant $c$ being determined by (5.2), and the function (2.9) appearing in the instanton $\Phi_{\mathrm{III}^{\prime}\left(D_{7}\right)}(t, \eta)$ that is normalized at a simple $P$-turning point $r$ of $\left(P_{\mathrm{III}^{\prime}\left(D_{7}\right)}\right)$ as

$$
\phi_{\mathrm{III}^{\prime}\left(D_{7}\right)}(\tilde{t})=\int_{r}^{t} \sqrt{F_{\mathrm{III}^{\prime}\left(D_{7}\right)}^{(1)}(t)} d t
$$


which satisfy the relations below:

(i) The function $t_{0}(\tilde{t})$ is independent of $\eta$ and satisfies

$$
\tilde{\phi}_{J}(\tilde{t})=\tilde{\phi}_{\mathrm{III}^{\prime}\left(D_{7}\right)}\left(t_{0}(\tilde{t})\right) \text {. }
$$

(ii) $d t_{0} / d \tilde{t}$ never vanishes on $\tilde{V}$

(iii) The function $x_{0}(\tilde{x}, \tilde{t})$ is also independent of $\eta$ and satisfies

$$
\begin{aligned}
x_{0}\left(\tilde{\lambda}_{0}(\tilde{t}), \tilde{t}\right) & =\lambda_{0}\left(t_{0}(\tilde{t}), c\right), \\
x_{0}(\tilde{a}(\tilde{t}), \tilde{t}) & =a\left(t_{0}(\tilde{t}), c\right) .
\end{aligned}
$$

Here $\lambda_{0}(t)$ and $a(t)$ are double and simple turning points of $\left(S L_{\mathrm{III}^{\prime}\left(D_{7}\right)}\right)$.

(iv) $\partial x_{0} / \partial \tilde{x}$ never vanishes on $\tilde{U} \times \tilde{V}$.

(v) $x_{1 / 2}$ and $t_{1 / 2}$ vanish identically.

(vi) The functions $\left\{x_{j / 2}(\tilde{x}, \tilde{t}, \eta)\right\}_{j=0}^{\infty}$ are single-valued in the annular domain $\tilde{U}$ as functions of $\tilde{x}$

(vii) The $\eta$-dependence of $x_{j / 2}$ and $t_{j / 2}(j \geq 2)$ is only through the instanton terms $\exp \left(\ell \tilde{\Phi}_{J}(\tilde{t}, \eta)\right)(\ell=j-2-2 m$ with $0 \leq m \leq j-2)$ that appear in the 2-parameter solution $\left(\tilde{\lambda}_{J}, \tilde{\nu}_{J}\right)$ of $\left(H_{J}\right)$.

(viii) The following relations hold:

$$
\begin{aligned}
x\left(\tilde{\lambda}_{J}(\tilde{t}, \eta ; \tilde{\alpha}, \tilde{\beta}), \tilde{t}, \eta\right)= & \lambda_{\mathrm{III}^{\prime}\left(D_{7}\right)}(t(\tilde{t}, \eta), \eta ; \alpha, \beta), \\
\tilde{Q}_{J}(\tilde{x}, \tilde{t}, \eta)= & \left(\frac{\partial x(\tilde{x}, \tilde{t}, \eta)}{\partial \tilde{x}}\right)^{2} Q_{\mathrm{III}^{\prime}\left(D_{7}\right)}(x(\tilde{x}, \tilde{t}, \eta), t(\tilde{t}, \eta), \eta) \\
& -\frac{1}{2} \eta^{-2}\{x(\tilde{x}, \tilde{t}, \eta) ; \tilde{x}\},
\end{aligned}
$$

where the 2-parameter solutions of $\left(H_{J}\right)$ and $\left(H_{\mathrm{III}^{\prime}\left(D_{7}\right)}\right)$ are substituted into $(\lambda, \nu)$ in the coefficients of $\tilde{Q}_{J}$ and $Q_{\mathrm{III}^{\prime}\left(D_{7}\right)}$, respectively, and $\{x(\tilde{x}, \tilde{t}, \eta) ; \tilde{x}\}$ denotes the Schwarzian derivative (2.41).

\section{§5.2. Construction of the top term of the transformation}

First we explain the construction of $t_{0}(\tilde{t})$ and $x_{0}(\tilde{x}, \tilde{t})$. In the proof we consider the case where the integration path (5.1) is taken along the path $\tilde{\Gamma}_{\tilde{t}, 1}$ in Figure 16(a). (This additional assumption is imposed just to fix ideas, and our discussion below is also applicable to the case where the integration path is taken along $\tilde{\Gamma}_{\tilde{t}, 2}$.) Then the definition (5.2) of the constant $c$ implies that

$$
\int_{\tilde{\Gamma}_{\tilde{t}, 1}} \sqrt{\tilde{F}_{J}^{(1)}(\tilde{t})} d \tilde{t}-\int_{\tilde{\Gamma}_{\tilde{t}, 2}} \sqrt{\tilde{F}_{J}^{(1)}(\tilde{t})} d \tilde{t}=\int_{\tilde{\Gamma}} \sqrt{\tilde{F}_{J}^{(1)}(\tilde{t})} d \tilde{t}=2 \pi i c .
$$


Let us construct $t_{0}(\tilde{t})$. Similarly to Section 4.2 , under the assumption that $\tilde{t}_{*}$ lies on the $P$-Stokes segment $\tilde{\Gamma}$, we can construct $t_{0}^{(k)}(\tilde{t})$ so that

$$
\tilde{\phi}_{J, k}(\tilde{t})=\phi_{\mathrm{III}^{\prime}\left(D_{7}\right), k}\left(t_{0}^{(k)}(\tilde{t})\right)
$$

for $k=1,2$, where

$$
\tilde{\phi}_{J, k}(\tilde{t})=\int_{\tilde{\Gamma}_{\tilde{t}, k}} \sqrt{\tilde{F}_{J}^{(1)}(\tilde{t})} d \tilde{t}, \quad \phi_{\mathrm{III}^{\prime}\left(D_{7}\right), k}(t)=\int_{\Gamma_{t, k}} \sqrt{F_{\mathrm{III}^{\prime}\left(D_{7}\right)}^{(1)}(t)} d t .
$$

Here the path $\Gamma_{t, k}$ for $\phi_{\mathrm{III}^{\prime}\left(D_{7}\right), k}(t)$ is a path from the $P$-turning point $r$ of $\left(P_{\mathrm{III}\left(D_{7}\right)}\right)$ to $t$ defined by the following rule. Note that, under Assumption 5.1(4), we have the following two possibilities for the Stokes geometry of $\left(S L_{J}\right)$ at $\tilde{t}_{*}$ (see Figure 18):

(A) The Stokes segment $\tilde{\gamma}_{2}$ follows the Stokes segment $\tilde{\gamma}_{1}$ in the counter-clockwise order near $\tilde{\lambda}_{0}\left(\tilde{t}_{*}\right)$.

(B) $\tilde{\gamma}_{2}$ follows $\tilde{\gamma}_{1}$ in the clockwise order near $\tilde{\lambda}_{0}\left(\tilde{t}_{*}\right)$.

Then we set

$$
\left(\Gamma_{t, 1}, \Gamma_{t, 2}\right)= \begin{cases}\left(\Gamma_{t}, \Gamma_{t}^{\prime}\right) & \text { when case }(\mathrm{A}) \text { happens, } \\ \left(\Gamma_{t}^{\prime}, \Gamma_{t}\right) & \text { when case }(\mathrm{B}) \text { happens }\end{cases}
$$

where $\Gamma_{t}$ and $\Gamma_{t}^{\prime}$ are the paths depicted in Figure $17(\mathrm{P})$. Moreover, the branch of $\sqrt{F_{\mathrm{III}^{\prime}\left(D_{7}\right)}^{(1)}(t)}$ in (5.11) is chosen so that the sign on the right-hand side of (3.17) is + (the orientation of $\Gamma$ is given appropriately):

$$
\int_{\Gamma_{t, 1}} \sqrt{F_{\mathrm{III}^{\prime}\left(D_{7}\right)}^{(1)}(t)} d t-\int_{\Gamma_{t, 2}} \sqrt{F_{\mathrm{III}^{\prime}\left(D_{7}\right)}^{(1)}(t)} d t=\int_{\Gamma} \sqrt{F_{\mathrm{III}^{\prime}\left(D_{7}\right)}^{(1)}(t)} d t=+2 \pi i c .
$$

This choice $(5.12)$ of $\Gamma_{t, 1}$ and $\Gamma_{t, 2}$ is essential in the construction of $x_{0}(\tilde{x}, \tilde{t})$.

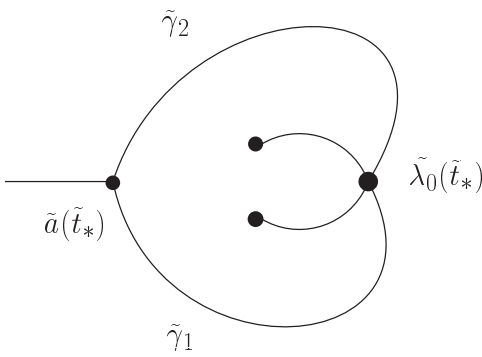

(A)

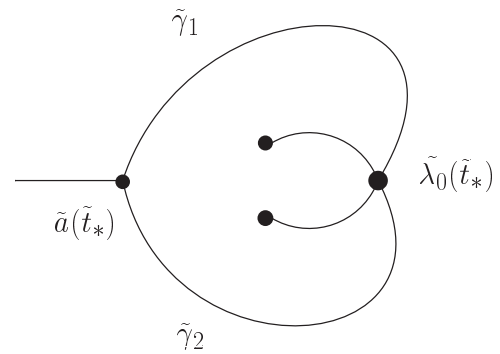

(B)

Figure 18. Two possibilities for adjacent Stokes segments of $\left(S L_{J}\right)$. 
Since the right-hand sides of (5.9) and (5.13) coincide, by the discussion of Section 4.2 we can show that $t_{0}^{(1)}(\tilde{t})=t_{0}^{(2)}(\tilde{t})$. We define $t_{0}(\tilde{t})=t_{0}^{(1)}(\tilde{t})=t_{0}^{(2)}(\tilde{t})$. Then, taking the path in (5.3) along $\Gamma_{1, t}$, we have (5.4).

Next we construct $x_{0}(\tilde{x}, \tilde{t})$. Set

$$
\left(\gamma_{1}, \gamma_{2}\right)= \begin{cases}\left(\gamma, \gamma^{\prime}\right) & \text { when case (A) happens, } \\ \left(\gamma^{\prime}, \gamma\right) & \text { when case (B) happens, }\end{cases}
$$

where $\gamma$ and $\gamma^{\prime}$ are the Stokes segments of $\left(S L_{\mathrm{III}^{\prime}\left(D_{7}\right)}\right)$ in Figure 17 (SL). Then, by (5.10) and our choice (5.12) of paths in (5.11), the discussion of Section 4.2 is also valid in this case because the relative location $\left(\tilde{a}, \tilde{\lambda}_{0}, \tilde{\gamma}_{1}, \tilde{\gamma}_{2}\right)$ of the simple turning point, the double turning point and the two Stokes segments of $\left(S L_{J}\right)$ completely coincides with those $\left(a, \lambda_{0}, \gamma_{1}, \gamma_{2}\right)$ of $\left(S L_{\mathrm{III}^{\prime}\left(D_{7}\right)}\right)$. Thus we can construct $x_{0}(\tilde{x}, \tilde{t})$ satisfying (5.5) and (5.6) and mapping the Stokes segments $\tilde{\gamma}_{1}$ and $\tilde{\gamma}_{2}$ to $\gamma_{1}$ and $\gamma_{2}$, respectively. Furthermore, $x_{0}(\tilde{x}, \tilde{t})$ becomes single-valued in an annular domain $\tilde{U}$ containing $\tilde{\gamma}_{1} \cup \tilde{\gamma}_{2}$ due to the following fact: At each turning point $\tilde{a}(\tilde{t})$ and $\tilde{\lambda}_{0}(\tilde{t})$, there exists a unique holomorphic function which maps $\tilde{\gamma}_{1}$ to $\gamma_{1}$ and it must coincide with $x_{0}(\tilde{x}, \tilde{t})$.

In what follows we choose the branches of $\sqrt{\tilde{Q}_{J, 0}(\tilde{x}, \tilde{t})}$ and $\sqrt{Q_{\operatorname{III}^{\prime}\left(D_{7}\right), 0}(x, t)}$ appearing in the proof so that

$$
\begin{aligned}
\int_{\tilde{\gamma}_{k}} \sqrt{\tilde{Q}_{J, 0}(\tilde{x}, \tilde{t})} d \tilde{x} & =\frac{1}{2} \int_{\tilde{\Gamma}_{\tilde{t}, k}} \sqrt{\tilde{F}_{J}^{(1)}(\tilde{t})} d \tilde{t} \\
\int_{\gamma_{k}} \sqrt{Q_{\mathrm{III}^{\prime}\left(D_{7}\right), 0}(x, t)} d x & =\frac{1}{2} \int_{\Gamma_{t, k}} \sqrt{F_{\mathrm{III}^{\prime}\left(D_{7}\right)}^{(1)}(t)} d t
\end{aligned}
$$

for $k=1,2$. In (5.15) and (5.16) the Stokes segments are directed from the simple turning point to the double turning point.

\section{§5.3. Construction of higher order terms of the transformation series and transformation of 2-parameter solutions}

Here we explain the construction of higher order terms of the transformation series. We note that most of the discussion in Section 4 is applicable in this case. The transformation series $x^{\text {pre }}(\tilde{x}, \tilde{t}, \eta)$ near the double turning point is constructed in the same manner as in Section 4.3, and the matching procedure of Section 4.4 is valid since we have only used the fact that "there is a Stokes segment of $\left(S L_{J}\right)$ connecting a simple turning point and the double turning point $\tilde{\lambda}_{0}\left(\tilde{t}_{*}\right)$ ". What we have to prove here is the single-valuedness of the higher order coefficients of formal series in the annular domain $\tilde{U}$ containing the union $\tilde{\gamma}_{1} \cup \tilde{\gamma}_{2}$ of two Stokes segments. 
Define

$$
\begin{aligned}
x^{\mathrm{pre}}(\tilde{x}, \tilde{t}, \eta) & =z_{\mathrm{III}^{\prime}\left(D_{7}\right)}^{-1}\left(\tilde{z}_{J}(\tilde{x}, \tilde{t}, \eta), s_{J}(\tilde{t}, \eta), \eta\right), \\
t^{\mathrm{pre}}(\tilde{t}, \eta) & =s_{\mathrm{III}^{\prime}\left(D_{7}\right)}^{-1}\left(\tilde{s}_{J}(\tilde{t}, \eta), \eta\right)
\end{aligned}
$$

in the same manner as in (4.24) and (4.25). Here we have fixed the correspondence of free parameters $(\tilde{\alpha}, \tilde{\beta})$ of $\left(\tilde{\lambda}_{J}, \tilde{\nu}_{J}\right)$ and $(\alpha, \beta)$ of $\left(\lambda_{\mathrm{III}^{\prime}\left(D_{7}\right)}, \nu_{\mathrm{III}^{\prime}\left(D_{7}\right)}\right)$ so that

$$
\tilde{E}_{J}(\tilde{\alpha}, \tilde{\beta})=E_{\mathrm{III}^{\prime}\left(D_{7}\right)}(\alpha, \beta),
$$

similarly to (4.21). Let $y^{(1)}(\tilde{x}, \tilde{t}, \eta)$ and $y^{(2)}(\tilde{x}, \tilde{t}, \eta)$ be formal series which transform $\left(S L_{J}\right)$ to $\left(S L_{\mathrm{III}^{\prime}\left(D_{7}\right)}\right)$ near the simple turning point $\tilde{a}(\tilde{t})$ in the sense of Lemma 4.5. Under our geometric assumption these two formal series coincide near $\tilde{x}=\tilde{a}(\tilde{t})$ for the following reason. Since the top term of $y_{0}^{(1)}(\tilde{x}, \tilde{t})$ of $y^{(1)}(\tilde{x}, \tilde{t}, \eta)$ maps the Stokes segment $\tilde{\gamma}_{1}$ of $\left(S L_{J}\right)$ to the Stokes segment $\gamma_{1}$ of $\left(S L_{\mathrm{III}^{\prime}\left(D_{7}\right)}\right)$ by definition, it also maps the other Stokes segment $\tilde{\gamma}_{2}$ to $\gamma_{2}$ simultaneously. Thus $y_{0}^{(1)}(\tilde{x}, \tilde{t})$ must coincide with $y_{0}^{(2)}(\tilde{x}, \tilde{t})$ near $\tilde{x}=\tilde{a}(\tilde{t})$, and hence the higher order terms also coincide, at least near $\tilde{x}=\tilde{a}(\tilde{t})$. In particular, their top terms also coincide with $x_{0}(\tilde{x}, \tilde{t})$ constructed in Section 5.2.

Furthermore, by the same argument as in Section 4.4 we can prove that all coefficients of $y^{(1)}(\tilde{x}, \tilde{t}, \eta)$ become holomorphic also at $\tilde{x}=\tilde{\lambda}_{0}(\tilde{t})$ and we have

$$
x^{\mathrm{pre}}(\tilde{x}, \tilde{t}, \eta)=y^{(1)}(\tilde{x}, \tilde{t}, \eta),
$$

after we choose the free parameters in $t^{\text {pre }}(\tilde{t}, \eta)$ appropriately. We denote by $t(\tilde{t}, \eta)$ the formal series $t^{\mathrm{pre}}(\tilde{t}, \eta)$ with parameters chosen appropriately in the above sense. Then there exists a domain $\tilde{U}^{\prime}$ near $\tilde{\lambda}_{0}(\tilde{t})$ in which all the coefficients of the two formal series $y^{(1)}(\tilde{x}, \tilde{t}, \eta)$ and $y^{(2)}(\tilde{x}, \tilde{t}, \eta)$ are holomorphic. We have to show that the analytic continuation of the coefficients of $y^{(1)}(\tilde{x}, \tilde{t}, \eta)$ along the Stokes segment $\tilde{\gamma}_{1}$ coincides with the analytic continuation of the coefficients of $y^{(2)}(\tilde{x}, \tilde{t}, \eta)$ along the Stokes segment $\tilde{\gamma}_{2}$ in the domain $\tilde{U}^{\prime}$. We will prove that the single-valuedness is guaranteed by our choice (5.2) of the constant $c$ in $\left(P_{\mathrm{III}^{\prime}\left(D_{7}\right)}\right)$ and $\left(S L_{\mathrm{III}^{\prime}\left(D_{7}\right)}\right)$.

Proposition 5.3. The analytic continuation of the coefficients of $y^{(1)}(\tilde{x}, \tilde{t}, \eta)$ along the Stokes segment $\tilde{\gamma}_{1}$ coincides with the analytic continuation of the coefficients of $y^{(2)}(\tilde{x}, \tilde{t}, \eta)$ along the Stokes segment $\tilde{\gamma}_{2}$ in the domain $\tilde{U}^{\prime}$ :

$$
y^{(1)}(\tilde{x}, \tilde{t}, \eta)=y^{(2)}(\tilde{x}, \tilde{t}, \eta) .
$$

Consequently, the coefficients of $y^{(1)}(\tilde{x}, \tilde{t}, \eta)$ and $y^{(2)}(\tilde{x}, \tilde{t}, \eta)$ are holomorphic and single-valued in $\tilde{x}$ on an annular domain $\tilde{U}$ containing $\tilde{\gamma}_{1} \cup \tilde{\gamma}_{2}$. 
Proof. In the proof we assume that case (A) in Figure 18 happens. (The discussion is also applicable to case (B).) Moreover, we will prove (5.21) for $\tilde{t}=\tilde{t}_{*}$. This is just for the sake of clarity, and our proof is also valid in a neighborhood $\tilde{V}$ of $\tilde{t}_{*}$. (We may take a smaller neighborhood $\tilde{V}$ of $\tilde{t}_{*}$.)

Let $\tilde{x}$ be a point in the domain $\tilde{U}^{\prime}$. Similarly to $(4.55)$, we have

$$
\int_{\tilde{\delta}_{\tilde{x}}^{(k)}} \tilde{S}_{J, \text { odd }}(\tilde{x}, \tilde{t}, \eta) d \tilde{x}=\left.\int_{\delta_{x}^{(k)}} S_{\mathrm{III}^{\prime}\left(D_{7}\right), \text { odd }}(x, t(\tilde{t}, \eta), \eta) d x\right|_{x=y^{(k)}(\tilde{x}, \tilde{t}, \eta)}
$$

for $k=1,2$. Here the integration path $\tilde{\delta}_{\tilde{x}}^{(k)}$ is depicted in Figure 19: it starts from the point on the second sheet of the Riemann surface of $\sqrt{Q_{J, 0}(\tilde{x}, \tilde{t})}$ corresponding to $\tilde{x}$, goes to the simple turning point $\tilde{a}\left(\tilde{t}_{*}\right)$ along the Stokes segment $\tilde{\gamma}_{k}$, encircles the simple turning point $\tilde{a}\left(\tilde{t}_{*}\right)$ and returns to the point corresponding to $\tilde{x}$ on the first sheet along the Stokes segment $\tilde{\gamma}_{k}$. (The wiggly line designates the branch cut for $\sqrt{Q_{J, 0}(\tilde{x}, \tilde{t})}$.) The path $\delta_{x}^{(k)}$ is defined in the same manner for $J=\operatorname{III}^{\prime}\left(D_{7}\right)$. Just as in (4.57), taking the difference of both sides of (5.22) for $k=1$ and $k=2$, we obtain

$$
\begin{aligned}
& \int_{\tilde{\delta}} \tilde{S}_{J, \text { odd }}(\tilde{x}, \tilde{t}, \eta) d \tilde{x}=\int_{\delta} S_{\mathrm{III}^{\prime}\left(D_{7}\right), \text { odd }}(x, t(\tilde{t}, \eta), \eta) d x \\
& \quad+\sum_{n=0}^{\infty} \frac{\partial^{n} S_{\mathrm{III}^{\prime}\left(D_{7}\right), \text { odd }}}{\partial x^{n}}\left(x_{0}, t(\tilde{t}, \eta), \eta\right) \frac{\left(y^{(2)}-x_{0}\right)^{n+1}-\left(y^{(1)}-x_{0}\right)^{n+1}}{(n+1) !} .
\end{aligned}
$$

Here $\tilde{\delta}=\tilde{\delta}_{+}+\tilde{\delta}_{-}$is the sum of two closed cycles $\tilde{\delta}_{+}$and $\tilde{\delta}_{-}$, where $\tilde{\delta}_{+}$(resp., $\left.\tilde{\delta}_{-}\right)$ encircles the double turning point $\tilde{\lambda}_{0}(\tilde{t})$ and all singular points contained in the domain $\tilde{W}$ (cf. Assumption 5.1(4)) in the clockwise (resp., counter-clockwise) direction on the first (resp., the second) sheet of the Riemann surface of $\sqrt{\tilde{Q}_{J, 0}(\tilde{x}, \tilde{t})}$ as indicated in Figure 19. The path $\delta$ for $J=\operatorname{III}^{\prime}\left(D_{7}\right)$ is defined in the same manner.
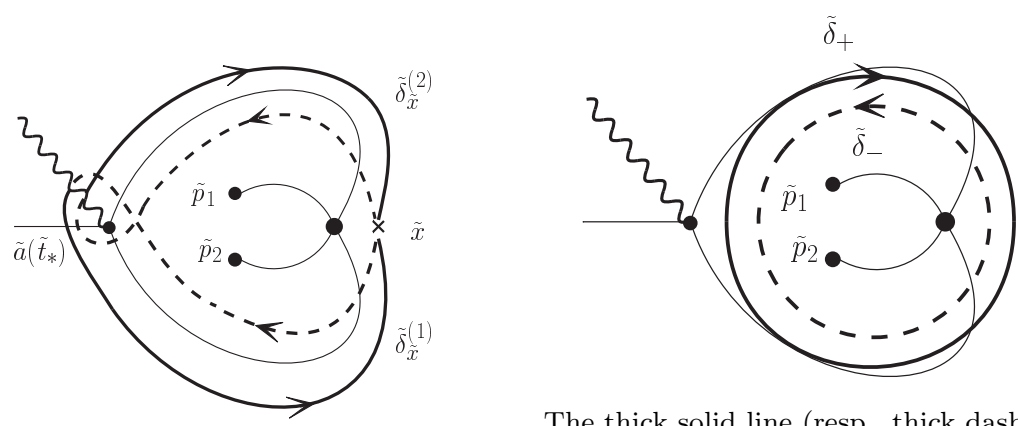

The thick solid line (resp., thick dashed line) designates the cycle $\tilde{\delta}_{+}$(resp., $\left.\tilde{\delta}_{-}\right)$

Figure 19. The cycles $\tilde{\delta}_{\tilde{x}}^{(k)}(k=1,2)$ and $\tilde{\delta}=\tilde{\delta}_{+}+\tilde{\delta}_{-}$. 
Under Assumption 5.1(5), there are no branch points of $\tilde{S}_{J, \text { odd }}(\tilde{x}, \tilde{t}, \eta)$ inside the closed cycle $\tilde{\delta}$. Thus the left-hand side of (5.23) can be written as

$$
\begin{aligned}
\int_{\tilde{\delta}} \tilde{S}_{J, \text { odd }}(\tilde{x}, \tilde{t}, \eta) d \tilde{x} & =\int_{\tilde{\delta}_{+}} \tilde{S}_{J, \text { odd }}(\tilde{x}, \tilde{t}, \eta) d \tilde{x}+\int_{\tilde{\delta}_{-}} \tilde{S}_{J, \text { odd }}(\tilde{x}, \tilde{t}, \eta) d \tilde{x} \\
& =4 \pi i \underset{\tilde{x}=\tilde{\lambda}_{0}(\tilde{t})}{\operatorname{Res}} \tilde{S}_{J, \text { odd }}(\tilde{x}, \tilde{t}, \eta) d \tilde{x}+4 \pi i R=\pi i \tilde{E}_{J}+4 \pi i R,
\end{aligned}
$$

where $R$ is the sum of the residues of $\tilde{S}_{J \text {,odd }}(\tilde{x}, \tilde{t}, \eta) d \tilde{x}$ at the singular points of $\tilde{Q}_{J, 0}(\tilde{x}, \tilde{t})$ in $\tilde{W}$. As in the proof of Lemma 4.7 we have (cf. (4.61))

$$
4 \pi i R=\eta \int_{\tilde{\delta}} \sqrt{\tilde{Q}_{J, 0}(\tilde{x}, \tilde{t})} d \tilde{x} .
$$

Here $\tilde{Q}_{J, 0}(\tilde{x}, \tilde{t})$ is holomorphic at $\tilde{x}=\tilde{\lambda}_{0}(\tilde{t})$. On the other hand, using (5.9) and (5.15), we have

$$
\oint_{\tilde{\delta}} \sqrt{\tilde{Q}_{J, 0}(\tilde{x}, \tilde{t})} d \tilde{x}=2\left(\int_{\tilde{\gamma}_{2}} \sqrt{\tilde{Q}_{J, 0}(\tilde{x}, \tilde{t})} d \tilde{x}-\int_{\tilde{\gamma}_{1}} \sqrt{\tilde{Q}_{J, 0}(\tilde{x}, \tilde{t})} d \tilde{x}\right)=-2 \pi i c .
$$

Then (5.24) yields

$$
\int_{\tilde{\delta}} \tilde{S}_{J, \text { odd }}(\tilde{x}, \tilde{t}, \eta) d \tilde{x}=\pi i \tilde{E}_{J}-2 \pi i c \eta .
$$

The same computation is also valid for $J=\operatorname{III}^{\prime}\left(D_{7}\right)$ and we obtain

$$
\int_{\delta} S_{\mathrm{III}^{\prime}\left(D_{7}\right), \text { odd }}(x, t, \eta) d x=\pi i E_{\mathrm{III}^{\prime}\left(D_{7}\right)}-2 \pi i c \eta
$$

from (5.13) and (5.16). Since the parameters $(\tilde{\alpha}, \tilde{\beta})$ and $(\alpha, \beta)$ are chosen as in (5.19), the equality (5.23) implies

$$
\sum_{n=0}^{\infty} \frac{\partial^{n} S_{\mathrm{III}^{\prime}\left(D_{7}\right), \text { odd }}}{\partial x^{n}}\left(x_{0}, t(\tilde{t}, \eta), \eta\right) \frac{\left(y^{(2)}-x_{0}\right)^{n+1}-\left(y^{(1)}-x_{0}\right)^{n+1}}{(n+1) !}=0 .
$$

Therefore, by induction we have the desired equality $(5.21)$ on $\tilde{U}^{\prime}$. Since $y^{(1)}(\tilde{x}, \tilde{t}, \eta)$ and $y^{(2)}(\tilde{x}, \tilde{t}, \eta)$ coincide at the simple turning point $\tilde{a}(\tilde{t})$ as is noted above, we have proved the single-valuedness of the transformation series.

Set

$$
x(\tilde{x}, \tilde{t}, \eta)=x^{\operatorname{pre}}(\tilde{x}, \tilde{t}, \eta) \quad\left(=y^{(1)}(\tilde{x}, \tilde{t}, \eta)=y^{(2)}(\tilde{x}, \tilde{t}, \eta)\right) .
$$

Then, since the equations (4.67) etc. also hold if we replace $\mathrm{II}$ by $\operatorname{III}^{\prime}\left(D_{7}\right)$, we have

$$
x\left(\tilde{\lambda}_{J}(\tilde{t}, \eta), \tilde{t}, \eta\right)=\lambda_{\mathrm{III}^{\prime}\left(D_{7}\right)}(t(\tilde{t}, \eta), \eta) .
$$

Thus we have proved all claims in Theorem 5.2. 


\section{Acknowledgements}

The author is grateful to Yoshitsugu Takei, Takahiro Kawai, Takashi Aoki, Tatsuya Koike, Shingo Kamimoto, Shinji Sasaki and Yasuhiro Wakabayashi for helpful advice. Many of the ideas of the proof of our main results come from the paper [KT2] of Kawai and Takei.

This research was supported by Research Fellowships of the Japan Society for Promotion for Young Scientists (grant no. 13J02831).

\section{References}

[AKT1] T. Aoki, T. Kawai and Y. Takei, The Bender-Wu analysis and the Voros theory, in Special functions (Okayama 1990), Springer, Tokyo, 1991, 1-29. Zbl 0782.35060 MR 1166808

[AKT2] WKB analysis of Painlevé transcendents with a large parameter. II. Multiplescale analysis of Painlevé transcendents, in Structure of solutions of differential equations, World Sci., 1996, 1-49. Zbl 0894.34050 MR 1445330

[AKT3] The Bender-Wu analysis and the Voros theory, II, in Algebraic analysis and around, Adv. Stud. Pure Math. 54, Math. Soc. Japan, Tokyo, 2009, 19-94. Zbl 1175.34112 MR 2499553

[DP] E. Delabaere and F. Pham, Resurgent methods in semi-classical asymptotics, Ann. Inst. Henri Poincaré 71 (1999), 1-94. Zbl 0977.34053 MR 1704654

[GIL] O. Gamayun, N. Iorgov and O. Lisovyy, How instanton combinatorics solves Painlevé VI, V and III's, J. Phys. A 46 (2013), 335203, 29 pp. Zbl 1282.34096 MR 3093009

[Iw1] K. Iwaki, Parametric Stokes phenomenon for the second Painlevé equation with a large parameter, Funkcial. Ekvac. 57 (2014), 173-243. Zbl 06366671 MR 3241905

[Iw2] Parametric Stokes phenomenon and the Voros coefficients of the second Painlevé equation, RIMS Kôkyûroku Bessatsu B40 (2013), 221-242. Zbl 1296.34178

[Iw3] Voros coefficients of the third Painlevé equation and parametric Stokes phenomena, arXiv:1303.3603[math CA] (2013).

[JMU] M. Jimbo, T. Miwa and K. Ueno, Monodromy preserving deformation of linear ordinary differential equations with rational coefficientns. I. General theory and $\tau$-function, Phys. D 2 (1981), 306-352. Zbl 1194.34167 MR 0630674

[KaKo] S. Kamimoto and T. Koike, On the Borel summability of 0-parameter solutions of nonlinear ordinary differential equations, RIMS Kôkyûroku Bessatsu B40 (2013), 191212. Zbl 06269173 MR 3156844

[KapKi] A. Kapaev and A. V. Kitaev, Passage to the limit $P_{2} \rightarrow P_{1}$, J. Math. Sci. 73 (1995), 460-467. Zbl 0834.34006 MR 1111905

[KT1] T. Kawai and Y. Takei, WKB analysis of Painlevé transcendents with a large parameter. I, Adv. Math. 118 (1996), 1-33. Zbl 0848.34005 MR 1375950

[KT2] WKB analysis of Painlevé transcendents with a large parameter. III. Local reduction of 2-parameter Painlveé transcendents, Adv. Math. 134 (1998), 178-218. Zbl 0901.34057 MR 1612399

[KT3] Algebraic Analysis of Singular Perturbation Theory, Transl. Math. Monogr. 227, Amer. Math. Soc., 2005. Zbl 1100.34004 MR 2182990 
[KT4] WKB analysis of higher order Painlevé equations with a large parameter Local reduction of 0 -parameter solutions for Painlevé hierarchies $\left(P_{J}\right)(J=\mathrm{I}$, II-1 or II-2), Adv. Math. 203 (2006), 636-672. Zbl 1108.34065

[Ki1] A. V. Kitaev, Turning points of linear systems and double asymptotics of the Painlevé transcendents, in Painlevé transcendents - their asymptotics and physical applications, NATO ASI Ser. B 278, Plenum, 1992, 81-96. Zbl 00929738 MR 1243682

[Ki2] Caustics in 1+1 integrable systems. J. Math. Phys. 35 (1994), 2934-2954. Zbl 0807.35135 MR 1275481

[Ki3] _ An isomonodromy cluster of two regular singularities, J. Phys. A 39 (2006), 12033-12072 (Sfb 288 preprint No. 149, 1994). Zbl 1116.34070 MR 2266211

[KiVa] A. V. Kitaev and A. H. Vartanian, Connection formulae for asymptotics of solutions of the degenerate third Painlevé equation: I, Inverse Problems 20 (2004), 1165-1206. Zbl 1064.34078 MR 2087985

[Ko] T. Koike, On the exact WKB analysis of second order linear ordinary differential equations with simple poles, Publ. RIMS Kyoto Univ. 36 (2000), 297-319. Zbl 0972.34078 MR 1753205

[OKSO] Y. Ohyama, H. Kawamuko, H. Sakai and K. Okamoto, Studies on the Painlevé equation. V. Third Painlevé equations of special type $\mathrm{P}_{\mathrm{III}}\left(D_{7}\right)$ and $\mathrm{P}_{\mathrm{III}}\left(D_{8}\right)$, J. Math. Sci. Univ. Tokyo 13 (2006), 145-204. Zbl 1170.34061 MR 2277519

[Ok] K. Okamoto, Isomonodromic deformation and Painlevé equations, and the Garnier system, J. Fac. Sci. Univ. Tokyo Sect. IA Math. 33 (1986), 575-618. Zbl 0631.34011 MR 0866050

[SS] H. Shen and H. J. Silverstone, Observations on the JWKB treatment of the quadratic barrier, in Algebraic analysis of differential equations, Springer, 2008, 237-250. Zbl 1145.34384 MR 2758911

[St] K. Strebel, Quadratic differentials, Ergeb. Math. Grenzgeb. 5, Springer, Berlin, 1984. Zbl 0547.30001 MR 0743423

[Ta] T. Takahashi, On the WKB theoretic structure of a Schrödinger operator with a Stokes curve of loop type, to appear in RIMS Kôkyûroku Bessatsu.

[T1] Y. Takei, On the connection formula for the first Painlevé equation from the viewpoint of the exact WKB analysis, RIMS Kôkyûroku 931 (1995), 70-99. MR 1404282

[T2] An explicit description of the connection formula for the first Painlevé equation, in Toward the exact WKB analysis of differential equations, linear or non-linear, Kyoto Univ. Press, 2000, 271-296. Zbl 0989.34075 MR 1770299

[T3] Sato's conjecture for the Weber equation and transformation theory for Schrödinger equations with a merging pair of turning points, RIMS Kôkyûroku Bessatsu B10 (2008), 205-224. Zbl 1170.34064 MR 2498081

[T4] On the turning point problem for instanton-type solutions of Painlevé equations, in Asymptotics in dynamics, geometry and PDEs; generalized Borel summation, Vol. II, CRM Ser. 12, Ed. Norm., Pisa, 2011, 255-274. Zbl 1246.34086 MR 3050814

[V] A. Voros, The return of the quartic oscillator: the complex WKB method, Ann. Inst. H. Poincaré Sect. A 39 (1983), 211-338. Z Zl 0526.34046 MR 0729194

[WT] H.Wakako and Y.Takei, Exact WKB analysis for the degenerate third Painlevé equation of type $\left(D_{8}\right)$, Proc. Japan Acad. Ser. A Math. Sci. 83 (2007), 63-68. Zbl 1152.34397 MR 2334372 